\title{
DUST EMISSION AND STAR FORMATION IN STEPHAN'S QUINTET
}

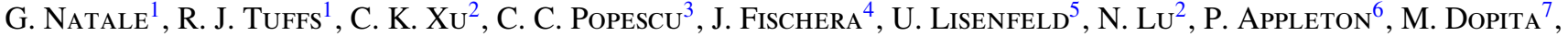

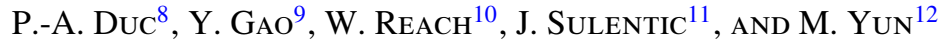 \\ ${ }^{1}$ Max Planck Institute für Kernphysik, Saupfercheckweg 1, D-69117 Heidelberg, Germany; giovanni.natale@ mpi-hd.mpg.de; richard.buffs@mpi-hd.mpg.de \\ ${ }^{2}$ Infrared Processing and Analysis Center, California Institute of Technology 100-22, Pasadena, CA 91125, USA \\ ${ }^{3}$ University of Central Lancashire, Preston, PR1 2HE, UK \\ ${ }^{4}$ Canadian Institute for Theoretical Astrophysics, University of Toronto, 60 Saint George Street, Toronto, ON, M5S 3H8, Canada \\ ${ }_{5}^{5}$ Department de Física Teórica y del Cosmos, Universidad de Granada, Granada, Spain \\ ${ }^{6}$ NASA Herschel Science Center, IPAC, California Institute of Technology, Pasadena, CA 91125, USA \\ ${ }^{7}$ Research School of Astronomy \& Astrophysics, The Australian National University, Cotter Road, Weston Creek, ACT 2611, Australia \\ ${ }^{8}$ Laboratoire AIM, CEA/DSM-CNRS-Université Paris Diderot, Dapnia/Service d'Astrophysique, CEA-Saclay, 91191 Gif-sur-Yvette Cedex, France \\ ${ }_{9}^{9}$ Purple Mountain Observatory, Chinese Academy of Sciences, 2 West Beijing Road, Nanjing 210008, China \\ ${ }^{10}$ Spitzer Science Center, IPAC, California Institute of Technology, Pasadena, CA 91125, USA \\ ${ }^{11}$ Instituto de Astrofísica de Andalucía, CSIC, Apdo. 3004, 18080, Granada, Spain \\ ${ }^{12}$ Department of Astronomy, University of Massachusetts, Amherst, MA 01003, USA \\ Received 2010 June 11; accepted 2010 October 6; published 2010 November 22
}

\begin{abstract}
We analyze a comprehensive set of MIR/FIR observations of Stephan's Quintet (SQ), taken with the Spitzer Space Telescope. Our study reveals the presence of a luminous $\left(L_{\mathrm{IR}} \approx 4.6 \times 10^{43} \mathrm{erg} \mathrm{s}^{-1}\right)$ and extended component of infrared dust emission, not connected with the main bodies of the galaxies, but roughly coincident with the X-ray halo of the group. We fitted the inferred dust emission spectral energy distribution of this extended source and the other main infrared emission components of SQ, including the intergalactic shock, to elucidate the mechanisms powering the dust and polycyclic aromatic hydrocarbon emission, taking into account collisional heating by the plasma and heating through UV and optical photons. Combining the inferred direct and dust-processed UV emission to estimate the star formation rate (SFR) for each source we obtain a total SFR for SQ of 7.5 $M_{\odot} \mathrm{yr}^{-1}, \operatorname{similar}$ to that expected for non-interacting galaxies with stellar mass comparable to the SQ galaxies. Although star formation in SQ is mainly occurring at, or external to the periphery of the galaxies, the relation of SFR per unit physical area to gas column density for the brightest sources is similar to that seen for star formation regions in galactic disks. We also show that available sources of dust in the group halo can provide enough dust to produce up to $L_{\mathrm{IR}} \approx 10^{42} \mathrm{erg} \mathrm{s}^{-1}$ powered by collisional heating. Though a minority of the total infrared emission (which we infer to trace distributed star-formation), this is several times higher than the X-ray luminosity of the halo, so could indicate an important cooling mechanism for the hot intergalactic medium (IGM) and account for the overall correspondence between FIR and X-ray emission. We investigate two potential modes of star formation in SQ consistent with the data, fueled either by gas from a virialized hot IGM continuously accreting onto the group, whose cooling is enhanced by grains injected from an in situ population of intermediate mass stars, or by interstellar gas stripped from the galaxies. The former mode offers a natural explanation for the observed baryon deficiency in the IGM of SQ as well as for the steep $L_{\mathrm{X}}-T_{\mathrm{X}}$ relation of groups such as SQ with lower velocity dispersions.
\end{abstract}

Key words: dust, extinction - galaxies: groups: individual (HGC92) - galaxies: interactions - galaxies: star formation - infrared: general - intergalactic medium

\section{INTRODUCTION}

Physical processes occurring in the environments of groups of galaxies play a fundamental role in determining the star formation history of the universe. Galaxy groups are associated with intermediate-mass dark matter halos (DMHs) which occupy a pivotal position in the formation of structures, acting as a center of aggregation of lower-mass DMHs and their associated galaxies while being the building blocks for the most massive clusters of galaxies that form at later epochs (Springel et al. 2005). In the present universe, about $50 \%$ of all stellar mass is contained within groups of total mass higher than $\log _{10} M / M_{\odot} \gtrsim 12.5$ (Eke et al. 2005). The group environment affects the modality in which baryonic gas in the intergalactic medium (IGM) is being converted into stars. Whereas at early epochs gas fueling of galaxies is thought to proceed via cold gas accretion in low-mass DMHs, this mechanism is predicted to be inhibited by the higher virial temperature of the IGM in high-mass DMHs hosting galaxy groups (Dekel \& Birnboim 2006). Furthermore, because of the high densities, galaxy-galaxy and galaxy-IGM interactions should become effective in removing interstellar gas from galaxies in groups, leading ultimately to a quenching of star formation in the already existing galaxies which have fallen into the groups. On the other hand, these same interactions lead to a chemical enrichment as the IGM becomes mixed with the stripped galaxian interstellar mediums which, potentially, could enhance the cooling of the IGM and its ability to accrete onto existing galaxies and to form new star-forming systems. The relative importance of all these processes in determining the star formation activity that is observed to be taking place in groups is an open question.

The Stephan's Quintet (SQ) ${ }^{13}$ compact group of galaxies presents a natural laboratory with which all these phenomena

\footnotetext{
13 Among the five galaxies that were observed for the first time by Édouard Stephan in 1877, NGC 7320 was later found to be a foreground dwarf galaxy. A nearby sixth galaxy, NGC 7320c, shows radial velocities compatible with $\mathrm{SQ}$. Therefore, the group is still a quintet even if the original definition has changed.
} 
affecting star formation in groups can be studied. As shown by (Trinchieri et al. 2005, hereafter T05) and (O'Sullivan et al. 2009, hereafter OS9), this group presents a diffuse halo of $\mathrm{X}$-ray emission extending in radius at least as far as $40 \mathrm{kpc}$ with the bulk of the gas radiating at temperatures $\approx 6 \times 10^{6} \mathrm{~K}$. Assuming hydrostatic equilibrium, this indicates a DMH mass of $\approx 10^{12} M_{\odot}$, intermediate between galaxies and clusters. The metal abundance of this hot gas is rather poorly constrained, consistent with a primordial and/or galaxian origin.

One galaxy, NGC 7318b, is apparently unbound, entering the group at a high relative velocity of $\approx 1000 \mathrm{~km} \mathrm{~s}^{-1}$ and colliding with the group IGM, as evidenced by a $\sim 32 \mathrm{kpc}$ north-south long ridge prominent in radio continuum (Xu et al. 2003), optical line emission (Xu et al. 1999), X-ray (Trinchieri et al. 2005), and recently also powerful mid-infrared rotational hydrogen lines (Appleton et al. 2006; Cluver et al. 2010). The galaxies of the group present extended tidal tails that have been used to constrain their recent interaction history (Moles et al. 1998; Sulentic et al. 2001). Potential consequences of these interactions is the presence of an active galactic nucleus (AGN) in the galaxy NGC 7319 (Huchra et al. 1982) and the presence of neutral and molecular gas located mainly outside the galaxies (Williams et al. 2002; Lisenfeld et al. 2002). UV observations with the Galaxy Evolution Explorer (GALEX) satellite have provided a detailed picture of the unobscured component of recent star formation in the group, including the tidal features (Xu et al. 2005)

In the context of understanding the physical processes controlling star formation in groups in general and in SQ in particular the FIR spectral region contains crucial information. Specifically, IR emission from dust is fundamental for an understanding of the amplitude and distribution of star formation since it traces the luminosity of young massive stars highly obscured by their parent molecular clouds at UV and optical wavelengths. The first infrared image of SQ, taken by the ISOCAM instrument on board the Infrared Space Observatory (ISO) revealed the presence of a starburst, SQ A, in the IGM (Xu et al. 1999), possibly triggered by the ongoing collision between the intruder galaxy and the IGM (Xu et al. 2003). The later GALEX imaging of SQ (Xu et al. 2005) also detected SQ A in the UV. Overall, the distribution of UV emission in SQ measured by GALEX shows that most of the recent unobscured star formation has preferentially occurred in the periphery regions or in the IGM. Further evidence for this is the discovery using the Hubble Space Telescope (HST) of widespread young star clusters distributed over the tidal debris and surrounding area by Gallagher et al. (2001). It is important to check whether this apparent shift from the main disk of the galaxies (where most star formation occurs in isolated galaxies, see, e.g., Leon et al. 2008) is also shown in the obscured component of star formation, and how the pattern of total star formation in the IGM is related to the morphological distribution of gas in the different temperature ranges.

A further motivation for studying SQ in the infrared are theoretical studies by Dwek et al. (1990) and Montier \& Giard (2004) which have predicted that even small amounts of dust in the hot virialized IGM could provide an important cooling mechanism via inelastic gas-grain collisions, with the radiation appearing in the FIR. This has prompted searches for an FIR counterpart to the X-ray-emitting intracluster medium (ICM) in several rich clusters (Stickel et al. 1998, 2002; Bai et al. 2007; Kitayama et al. 2009) which however have thus far yielded no unambiguous detection. This may be attributed to the very low abundance of grains predicted on the basis of realistic estimates of sources and sinks of grains in the IGM (Popescu et al. 2000), the expected similarity of the FIR colors of the collisionally heated dust emission component with photon-heated diffuse dust in foreground cirrus (Popescu et al. 2000), and the problem of spatial confusion with star-forming galaxies in the cluster (Quillen et al. 1999). SQ is a good object to search for this phenomena since its angular size is small enough for accurate photometric measurements of extended emission and, at the same time, it is possible to separate emission from discrete starforming sources, such as the constituent galaxies and objects like SQ A. Finally, the detection of dust FIR emission, combined with gas mass measurements to obtain the dust-to-gas ratio, is a sensitive way of probing the metallicity, and hence the origin, of the various gas components seen in the group in situations where optical nebular line diagnostic are weak or absent.

The most extensive previous study of the FIR emission from SQ was that by $\mathrm{Xu}$ et al. (2003) in the wavelengths range $11-100 \mu \mathrm{m}$ using the $I S O$. Apart from strong detections of the AGN galaxy NGC 7319 and the foreground galaxy NGC 7320, significant detections were obtained in the FIR of the intergalactic star formation region SQ A and the source SQ B located on the tidal feature associated with NGC 7319. Intriguingly, the ISO images also hinted at the presence of FIR emission associated with the X-ray-emitting shock ridge, which was argued to be evidence for collisionally heated dust embedded in the hot shocked gas.

In this paper, we present deep imaging of the FIR emission from SQ taken with the Spitzer Space Telescope. These data have superior angular resolution and sensitivity compared to the previous $I S O$ study and moreover extend the wavelength coverage longward to $160 \mu \mathrm{m}$, thus providing much deeper investigation of any cold dust components, embracing the expected spectral peak of the emission from photon- and collisional-heated emissions. The data are used to characterize the pattern of star formation in SQ and to investigate possible infrared counterparts of the X-ray-emitting structures. The Spitzer observations and data reduction are described in Section 2, where we also collate multiwavelength data needed for our analysis. In Section 3, we describe the morphology of the dust emission of SQ in relation to the multiwavelength data set and also describe a novel fitting technique to the lower angular resolution FIR maps which we use to separate the emission from the main emitting structures. After extracting photometry from these structures across MIR/FIR range in Section 4, we fit the MIR/FIR SEDs with models in Section 5 to elucidate the mechanisms powering the observed dust emission of the sources in SQ. This information is then used to quantify star formation rates (SFRs) in Section 6 where we also quantify corresponding gas reservoir available to fuel the star formation. In Section 7, we quantitatively discuss the nature of star formation in and outside the constituent galaxies of SQ, together with the related question of the extent to which collisional heating plays a role in determining the thermodynamic properties of the IGM in SQ. In this paper, we assume a distance from the group equal to $94 \mathrm{Mpc}$, corresponding to a systemic velocity of $v=6600 \mathrm{~km} \mathrm{~s}^{-1}$ and assuming $H_{0}=70 \mathrm{~km} \mathrm{~s}^{-1} \mathrm{Mpc}^{-1}$.

\section{OBSERVATIONS AND DATA REDUCTION}

In this work, we have used Spitzer maps from two Guest Observer (GO) programs making use of continuum data at 24, 70, and $160 \mu \mathrm{m}$ from the MIPS instrument (Rieke et al. 2004). From the GO No. 40142 (PI: P. Appleton) we took the $24 \mu \mathrm{m}$ 

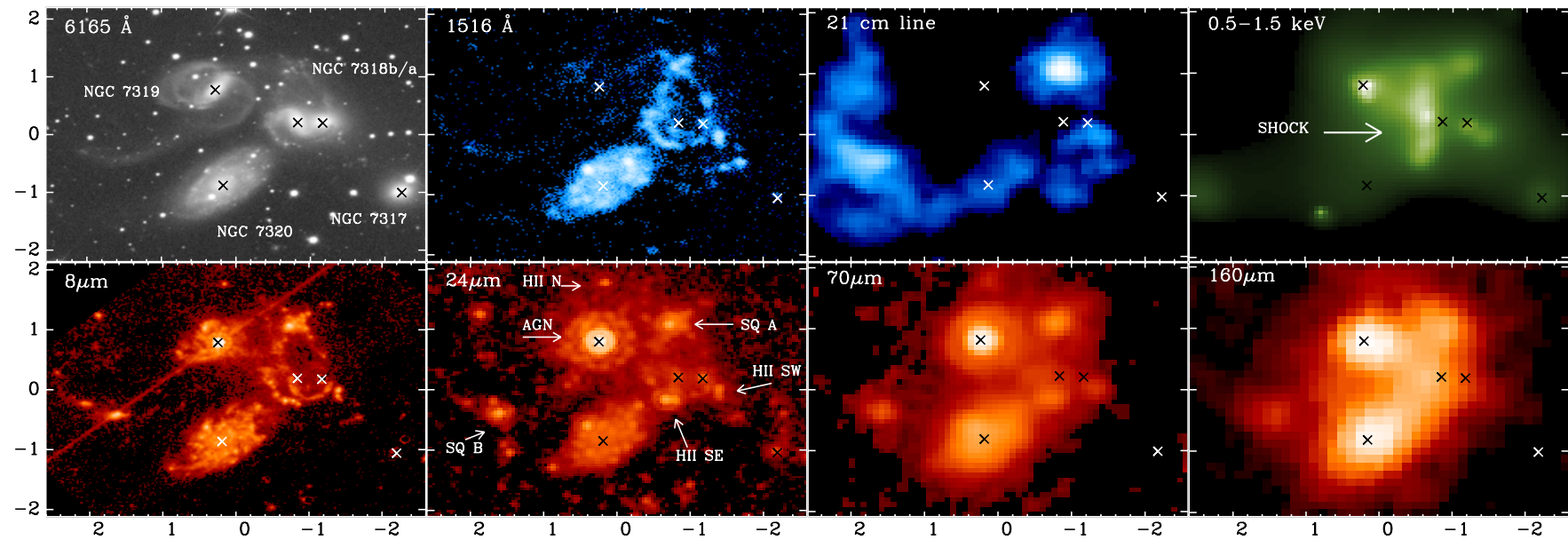

Figure 1. Stephan's Quintet multiwavelength data. Upper row from left to right: SDDS $r$ band, GALEX FUV, VLA radio, and XMM-Newton soft X-ray. Lower row: Spitzer IRAC $8 \mu \mathrm{m}$ and MIPS 24,70,160 $\mu \mathrm{m}$. Crosses on the maps identify the galaxy centers. The position $(0,0)$ coincides with R.A. $=22^{\mathrm{h}} 36^{\prime} 02^{\prime \prime} .4$ decl. $=+33^{\circ} 57^{\prime} 46^{\prime \prime}$. The units on the axis are arcmin. At the distance of SQ (94 Mpc) every arcmin corresponds to 24 kpc. Note that NGC 7320 is a foreground galaxy at the distance of $10 \mathrm{Mpc}$ and its $\mathrm{H}$ I distribution, not shown in this figure, can be found in Williams et al. (2002).

map and from the GO No. 3440 (PI: K. Xu) the 70 and $160 \mu \mathrm{m}$ maps. In addition, an image of SQ at $8 \mu \mathrm{m}$, taken with the IRAC instrument (Fazio et al. 2004) and downloaded from the Spitzer Science Center (SSC) archive, was also used. In the following, we provide details of the data reduction for the Spitzer FIR maps. The $24 \mu \mathrm{m}$ map has been already presented by Cluver et al. (2010) and we refer to that paper for technical details about the data preparation.

\subsection{MIPS $70 \mu \mathrm{m}$ and $160 \mu \mathrm{m}$ Data Reduction}

The MIPS $70 \mu \mathrm{m}$ and $160 \mu \mathrm{m}$ data are from Spitzer pipeline version S11.0.2. In the pipeline default mosaic images, there are a few residual instrumental artifacts that are particularly noticeable at $70 \mu \mathrm{m}$. Because of this, these pipeline images were not used in this work. In their place, new images were used in which the instrumental artifacts were reduced by performing additional data reduction steps on the "basic calibrated data" (BCD) frames.

Specifically, for the $70 \mu \mathrm{m}$ data, we observed an overall signal drift in time. To remove this, we masked out those pixels of each of the 468 non-stim BCD frames that are within a radius of $125^{\prime \prime}$ of the SQ center $($ R.A. $=339$ d.0181, decl. $=33$ d.969183; J2000). For each BCD frame, a median was calculated from all unmasked pixels. These medians were plotted as a function of the BCD frame index (1-468). The resulting plot shows a clear discontinuity at frame index 313 . We fit the sections prior to and post this discontinuity separately with a cubic spline function of order 1 or 2 . The two fitted curves were connected to form one curve covering all the $\mathrm{BCD}$ frames. After being normalized by its mean, this curve was divided into the index-ordered $\mathrm{BCD}$ frames to remove the signal drift in time. The next step was to create a sky flat image by median filtering only unmasked data for a given detector pixel. The resulting sky flat image was normalized by its mean, and subsequently divided into each unmasked BCD frames. Finally, we used Spitzer MOPEX tool to mosaic these improved BCD frames into our final image used in this paper. With a much flatter sky background, our own mosaic image is significantly better than the pipeline counterpart.

For the MIPS $160 \mu \mathrm{m}$ data, a similar procedure was used with all 522 non-stim BCD frames. In this case, the detector signal drifts in time differ significantly among individual readout modules. As a result, our signal drift removal was attempted on per readout module basis.

Background subtraction has been performed by fitting a tilted plane to the maps, after masking a large area covering the main group and, in the case of the $160 \mu$ m map, the galaxy NGC 7320c (that lies outside the field of view shown in Figure 1). The total area used to estimate the background is about $50 \%$ of the entire maps at both wavelengths. These areas are well outside the group emission, allowing good background measurements.

\subsection{Collected Multiwavelength Data}

In this paper, we have also made use of a large set of multiwavelength data to derive physical quantities or for morphological comparison with the emission seen on the infrared maps. Specifically, we utilized the Sloan Digital Sky Survey (SDSS) maps of SQ, obtained from the SDSS Data Archive (data release 7), the GALEX FUV map, which have been presented in Xu et al. (2005), the XMM-Newton soft X-ray map from Trinchieri et al. (2005), the Very Large Array (VLA) $21 \mathrm{~cm}$ line map from Williams et al. (2002), the $\mathrm{H} \alpha$ maps from Xu et al. (1999), the IRAC $3.6 \mu \mathrm{m}$ map, obtained from the SSC archive, and the CO radio observations presented in Lisenfeld et al. (2002).

\section{MORPHOLOGY OF DUST EMISSION IN SQ}

In Figure 1 (lower panel), we show all the Spitzer broad bands (from IRAC and MIPS) where the signal is dominated by dust emission: ${ }^{14} 8 \mu \mathrm{m}, 24 \mu \mathrm{m}, 70 \mu \mathrm{m}$, and $160 \mu \mathrm{m}$. These can be compared with the upper panel showing the SDSS $r$-band map, the GALEX FUV map, the VLA radio $21 \mathrm{~cm}$ line map, and the $X M M$-Newton soft X-ray map. On each map, crosses identify the galaxy centers.

The IR morphology of the galaxies seen in the IR maps is markedly different from the optical morphology. This is true not only for the early-type galaxies but also for the late-type galaxies which exhibit remarkably little infrared emission from the main

\footnotetext{
14 Throughout this paper all the $8 \mu \mathrm{m}$ images presented have had the stellar component of the emission subtracted using the relation

$F_{v}(8 \mu \mathrm{m}$, dust $)=F_{v}(8 \mu \mathrm{m})-0.232 F_{v}(3.6 \mu \mathrm{m})$ (Helou et al. 2004). The $8 \mu \mathrm{m}$ Spitzer band also contains emission from rotational hydrogen lines which however we estimate in Section 5 to be unimportant in relation to the $\mathrm{PAH}$ and dust continuum emission.
} 
bodies of the galaxies. The only exception is the emission from the foreground galaxy NGC 7320 which is quite symmetrical in all the Spitzer bands, having a filled disk of emission commonly seen in local universe field galaxies, compatible with the optical/ UV appearance not showing any sign of interactions (compatible with its not being a member of SQ).

The infrared emission from NGC 7319 is dominated by an unresolved nuclear source, presumably from the Seyfert 2 AGN, that is particularly prominent at 24 and $70 \mu \mathrm{m}$. At $8 \mu \mathrm{m}$, one can also clearly see emission from the disk of the AGN host galaxy. As can be seen from the MIR maps, dust emission from compact star formation regions is detected all over the group and especially on the elongated features of the intruder galaxy NGC 7318b which are most prominently delineated in the UV. Here, the similarity is strongest between the UV and the $8 \mu \mathrm{m}$ band though the most prominent discrete sources are also clearly seen at 24 and $70 \mu \mathrm{m}$. The most prominent such MIR/FIR source is SQ A, the star formation region located to the north of NGC 7318b, already detected by ISO, which can also be seen at $160 \mu \mathrm{m}$. From optical spectra (Xu et al. 2003) and radio observations (Lisenfeld et al. 2002; Williams et al. 2002), it is known that star formation in this region is associated with gas at radial velocities corresponding to both the intruder galaxy and the IGM of the group. Several further compact star formation regions are located on the southern arms of NGC 7318b. The brighter sources, H II-SE and H II-SW, are also detected on the $70 \mu \mathrm{m}$ map but not clearly seen on the lower resolution $160 \mu \mathrm{m}$ map. Two other bright MIR/FIR-emitting regions detected on the Spitzer maps are SQ B, a star formation region located on the optical "young" tidal tail (see Sulentic et al. 2001), and a source, H II-N, located about 40 " toward the north of the AGN galaxy.

There is no clear morphological counterpart in the infrared to the shock region, defined here by the ridge of emission that can be seen on the X-ray map. Nevertheless, the $70 \mu \mathrm{m}$ map and more particularly the $160 \mu \mathrm{m}$ map show enhanced emission toward the peak of the X-ray emission. The ratio of the MIR 8 and $24 \mu \mathrm{m}$ emission to the $160 \mu \mathrm{m}$ emission at the same position appears rather low compared to the discrete sources associated with star formation such as SQ A. A previously undetected feature is an extended FIR emission component, spatially coincident with the main part of the group X-ray halo (as defined in Trinchieri et al. 2005). The appearance of the corresponding MIR emission on the higher resolution 8 and $24 \mu \mathrm{m}$ images suggests that the extended FIR emission may at least in part be clumpy rather than uniform, possibly indicating the presence of faint star formation regions far away from the centers of the galaxies.

In order to quantify the morphology and brightness of the FIR emission seen toward the X-ray-emitting halo and shock regions, it is necessary to subtract from the FIR maps the most prominent discrete sources associated with star formation regions and galaxies. To do this, we devised an FIR source fitting technique which we describe in Section 3.1. This source fitting technique also serves to fix the photometry and the extent of discrete sources, information that cannot be directly extracted from the maps due to the unknown level of mutual confusion.

\subsection{The FIR Map Fitting Technique}

The fitting technique models a preselected set of the most prominent discrete sources as a sum of elliptical Gaussian convolved with the point-spread function (PSF). Seven parameters are calculated for each source: amplitude, the peak coordinates, the two Gaussian widths, the axis rotation angle, and the local background (included to avoid removal of any diffuse emission components). The fit is performed simultaneously for the 10 brightest sources seen on the $70 \mu \mathrm{m}$ maps (see Figure 2): five compact sources (SQ A, H II-SE, H II-SW, SQ B, H II-N), two sources to fit the emission from the AGN galaxy NGC 7319 (one for the central emission and one for a peripherical star formation region visible after the removal of the first component), two for the fit of the foreground galaxy NGC 7320 (one for the fit of the diffuse emission and one for a compact source), and one to fit the emission peaked in the middle of the shock region. The fit to the $70 \mu \mathrm{m}$ map is performed first, keeping all the fitting parameters as free variables. This is followed by a constrained fit to the $160 \mu \mathrm{m}$ map in which the relative position of the sources are fixed to the values obtained at $70 \mu \mathrm{m}$. In addition, a further constraint was that we kept the same shape and axis orientation for the five compact sources, as inferred by the $70 \mu \mathrm{m}$ fit, allowing only a size change (to take into account the potentially more extended distribution of cold dust emission). This strategy was adopted because the higher resolution $70 \mu \mathrm{m}$ map places the strongest constraints to the position and morphology of sources in the FIR. We did not use the highest resolution $8 \mu \mathrm{m}$ map for this purpose since, whereas the emission detected at 70 and $160 \mu \mathrm{m}$ is produced by the same kind of solid dust grains, the emission at $8 \mu \mathrm{m}$ is dominated by line emission from polycyclic aromatic hydrocarbon (PAH) molecules. Model images from the fitting technique are shown in Figures 2 and 3. The best-fit parameters and the inferred total flux densities are shown in Table 1. At both $70 \mu \mathrm{m}$ and $160 \mu \mathrm{m}$, the best-fit model images are remarkably similar to the original maps. On the "deconvolved" maps in each figure, it is possible to see the contribution from each Gaussian to the final map. Interestingly enough, the source at the position of the shock is much more predominant at $160 \mu \mathrm{m}$ than at $70 \mu \mathrm{m}$, confirming the original impression that the emission in the shock region is brighter at $160 \mu \mathrm{m}$. It is also noteworthy that the position angle of the model source at $160 \mu \mathrm{m}$ is aligned with the north-south orientation of the X-ray-emitting ridge whereas at $70 \mu \mathrm{m}$ no such alignment is apparent. At both 70 and $160 \mu \mathrm{m}$, the east-west width of the fitted elliptical Gaussians at the shock position (FWHM $\left.\approx 60^{\prime \prime}\right)$ is larger than that of the X-ray shock ridge $\left(\mathrm{FWHM} \approx 20^{\prime \prime}\right)$. This indicates that the integrated emission is not necessarily entirely composed of emission from the shock ridge. A full description of the source fitting technique is given in Appendix A.

\subsection{The FIR Residual Maps}

To understand how the emission peaked in the shock region and the extended emission are distributed on the maps, we created FIR residual maps where the emission from all the sources fitted by the FIR map fitting technique, with the exception of the source associated with the shock, have been subtracted. The contours of these FIR residual maps are shown in Figure 4 overlaid on Hi, X-ray, and FUV maps. As one can see, the emission on the FIR residual maps is uncorrelated and perhaps even anticorrelated with the Hi distribution but well correlated with the soft X-ray flux. The FIR emission, as already seen on the original maps, peaks in the middle of the shock region and its overall extent is similar to the X-ray halo emission (similar to what was seen in the low excitation pure rotational lines of $\mathrm{H}_{2}$ by Cluver et al. 2010). At first sight, this finding supports the idea that collisional heating is producing the observed FIR emission. However, a large part of the residual FIR emission covers areas emitting significant 


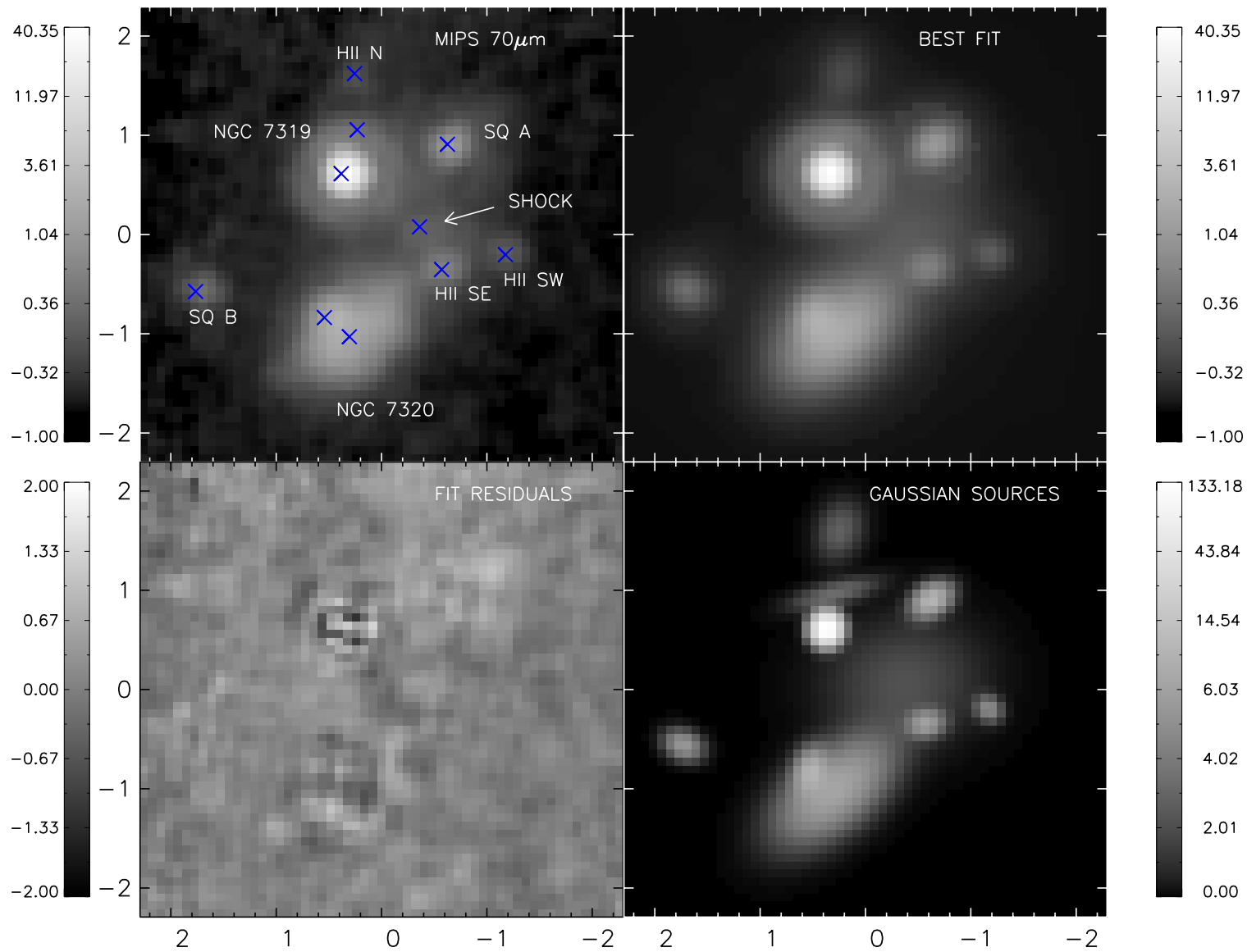

Figure 2. Fit results for the $70 \mu \mathrm{m}$ map. Top left: original $70 \mu \mathrm{m}$ map; top right: best-fit map; bottom left: fit residuals; bottom right: "deconvolved" map. The crosses on the original map identify the centers of the fitted sources. Units of the values aside the color bars are $\mathrm{MJy} \mathrm{sr}^{-1}$. Note: the fit residual map, shown on the bottom left panel, has all the fitted sources subtracted. It differs from the $70 \mu \mathrm{m}$ "FIR residual map," whose contours are shown in the upper panels of Figure 4, where the SHOCK source has not been subtracted.

Table 1

Results of the FIR Map Fitting

\begin{tabular}{lcccccc}
\hline \hline \multicolumn{1}{c}{ Source } & $\begin{array}{c}\text { R.A. } \\
(\mathrm{h} \mathrm{m} \mathrm{s})\end{array}$ & $\begin{array}{c}\text { Decl. } \\
\left({ }^{\prime}{ }^{\prime \prime}\right)\end{array}$ & $\begin{array}{c}\Delta 70 \mu \mathrm{m} \\
(\operatorname{arcs})\end{array}$ & $\begin{array}{c}\Delta_{160 \mu \mathrm{m}} \\
(\operatorname{arcs})\end{array}$ & $\begin{array}{c}F_{70 \mu \mathrm{m}} \\
(\mathrm{mJy})\end{array}$ & $\begin{array}{c}F_{160 \mu \mathrm{m}} \\
(\mathrm{mJy})\end{array}$ \\
\hline SQ A & 223558.81 & 335851.1 & 16 & 15 & $134 \pm 15$ & $265 \pm 86$ \\
H II-SE & 223559.08 & 335734.0 & 14 & 40 & $61 \pm 9$ & $186 \pm 140$ \\
H II-SW & 223556.10 & 335743.1 & 11 & ND & $31 \pm 5$ & ND \\
SQ B & 223610.54 & 335720.5 & 14 & 36 & $63 \pm 8$ & $302 \pm 34$ \\
N7319 & 22363.76 & 335833.0 & 12 & 30 & $616 \pm 62$ & $1192 \pm 130$ \\
N7319 (H II) & 22363.01 & 33590.0 & 24 & ND & $50 \pm 8$ & ND \\
N7320 & 22363.38 & 335652.7 & 44 & 48 & $716 \pm 73$ & $1899 \pm 196$ \\
N7320 (H II) & 22364.54 & 33574.5 & 16 & ND & $111 \pm 13$ & ND \\
H II-N & 22363.13 & 335934.6 & 23 & 42 & $52 \pm 6$ & $98 \pm 41$ \\
SHOCK $^{\text {a }}$ & 22360.10 & 33580.3 & 61 & 58 & $258 \pm 30$ & $1218 \pm 230$ \\
& & & & & &
\end{tabular}

Notes. Column 1: FIR source; Columns 2 and 3: source center R.A. and decl. coordinates; Columns 4 and 5: averaged FWHMs of the sources at $70 \mu \mathrm{m}$ and $160 \mu \mathrm{m}$; Columns 6 and 7: total flux densities at $70 \mu \mathrm{m}$ and $160 \mu \mathrm{m}$. Some of the sources are not detected at $160 \mu \mathrm{m}$ (ND: "non-detected").

a The SHOCK source on this table refers only to the Gaussian used to fit the emission centered in the middle of the shock region. Therefore, the quoted fluxes do not represent the actual fluxes coming from that area. The emission from the shock region is given in the Table 2.

luminosity at $\mathrm{UV}$ wavelengths. The presence of these radiation sources complicates the interpretation of the dust emission in the shock region as well as for the extended emission (see Section 7).

\section{SQ SPITZER MAP PHOTOMETRY}

Precise photometry of all these different emitting regions in SQ is required in order to elucidate the physical mechanisms that power dust emission and the related scientific implications. In the FIR, the photometry is derived from the source fitting procedure (for the discrete sources) and from the residual maps (for the shock and the extended emission components). In the MIR, the high resolution of the MIR 8 and $24 \mu \mathrm{m}$ maps allows a straightforward extraction of fluxes of the corresponding regions in SQ using aperture photometry. All results are summarized in Table 2. 


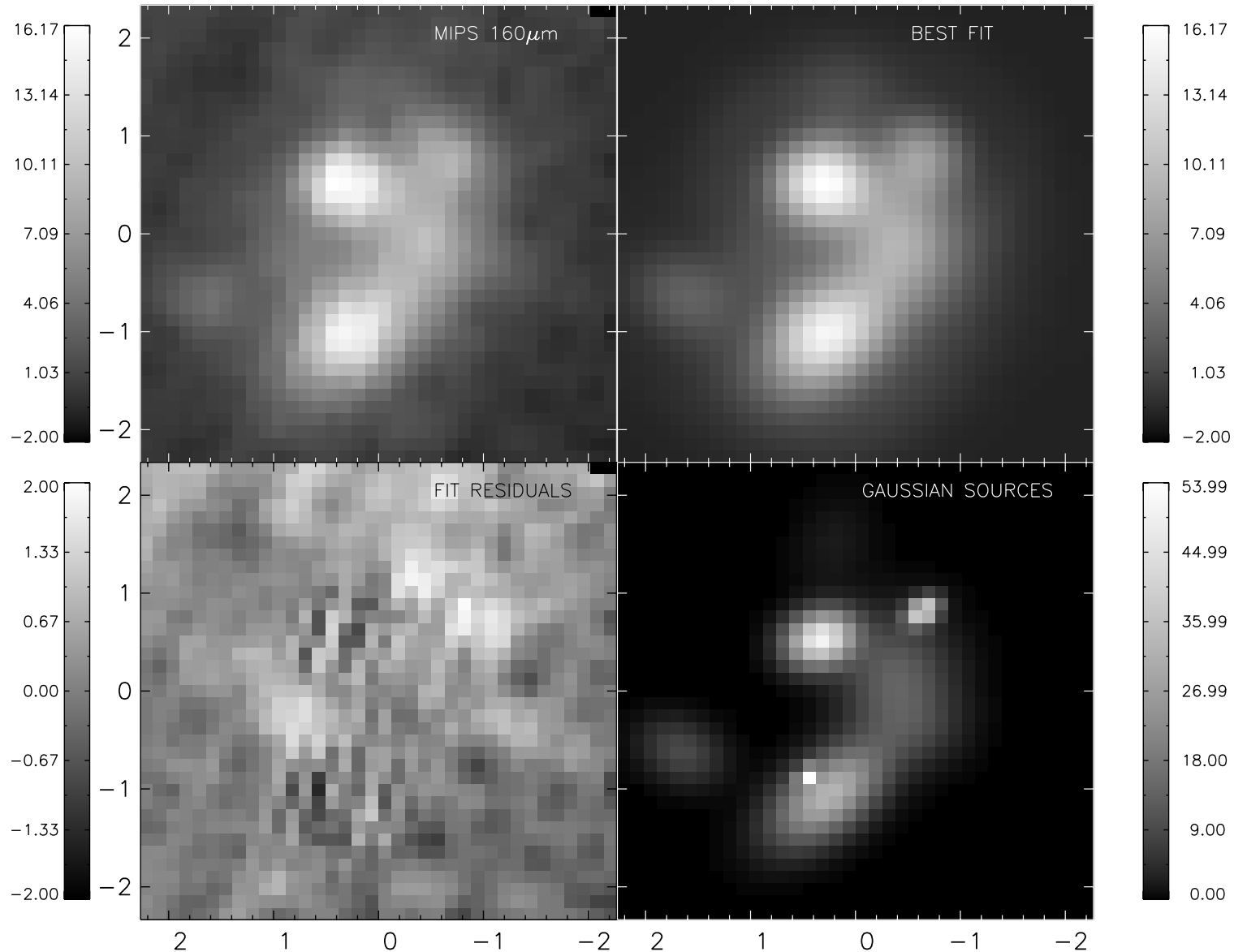

Figure 3. Fit results for the $160 \mu \mathrm{m}$ map. Top left: original $160 \mu \mathrm{m}$ map; top right: best-fit map; bottom left: fit residuals; bottom right: "deconvolved" map. Units of the values aside the color bars are $\mathrm{MJy} \mathrm{sr}^{-1}$. Note: the fit residual map, shown on the bottom-left panel, has all the fitted sources subtracted. It differs from the $160 \mu \mathrm{m}$ "FIR residual map," whose contours are shown in the lower panels of Figure 4, where the SHOCK source has not been subtracted.
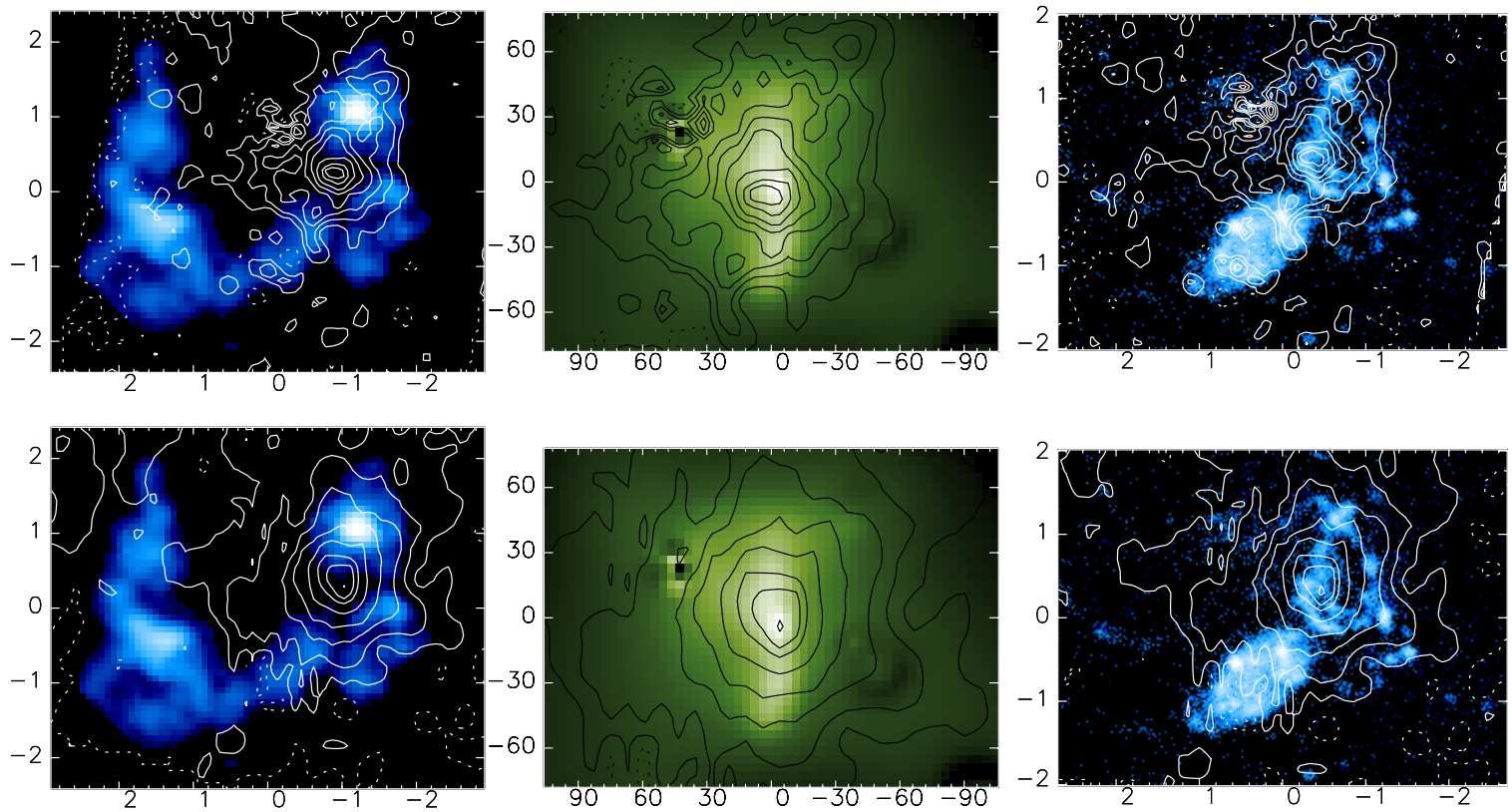

Figure 4. FIR residual maps (see Section 3.2) contours overlaid on Hi (left panels), X-ray (middle panels), and FUV maps (right panels). The contours in the upper row are from the $70 \mu \mathrm{m}$ residual map while those in the lower row are from the $160 \mu \mathrm{m}$ residual map. The $70 \mu \mathrm{m}$ residual map contour levels are $-0.3,0.3,0.6,0.9,1.2,1.5,1.8,2.1,2.25 \mathrm{MJy} \mathrm{sr}^{-1}$ and those for the $160 \mu \mathrm{m}$ residual map are $-0.4,0.4,1.2,2.4,3.6,4.8,6.0,6.6,7.2 \mathrm{MJy} \mathrm{sr}^{-1}$ (the dashed lines are the negative contours, corresponding to $-1 \sigma$ level). The field of view is different in the three maps. The coordinates corresponding to $(0,0)$ are for the $\mathrm{HI}$ map R.A. $=22^{\mathrm{h}} 36^{\prime} 04^{\prime \prime} .1$ and decl. $=+33^{\circ} 57^{\prime} 47^{\prime \prime} .2$, for the X-ray map R.A. $=22^{\mathrm{h}} 35^{\prime} 59^{\prime \prime} \cdot 7$ and decl. $=+33^{\circ} 58^{\prime} 05^{\prime \prime} \cdot 9$, for the FUV map R.A. $=22^{\mathrm{h}} 36^{\prime} 01^{\prime \prime} .6$ and decl. $=+33^{\circ} 57^{\prime} 42^{\prime \prime}$. 1 . The axis units are arcminutes for the H I and FUV maps and arcseconds for the X-ray map. Note that point sources have been removed from the X-ray map (see Figure 1). 


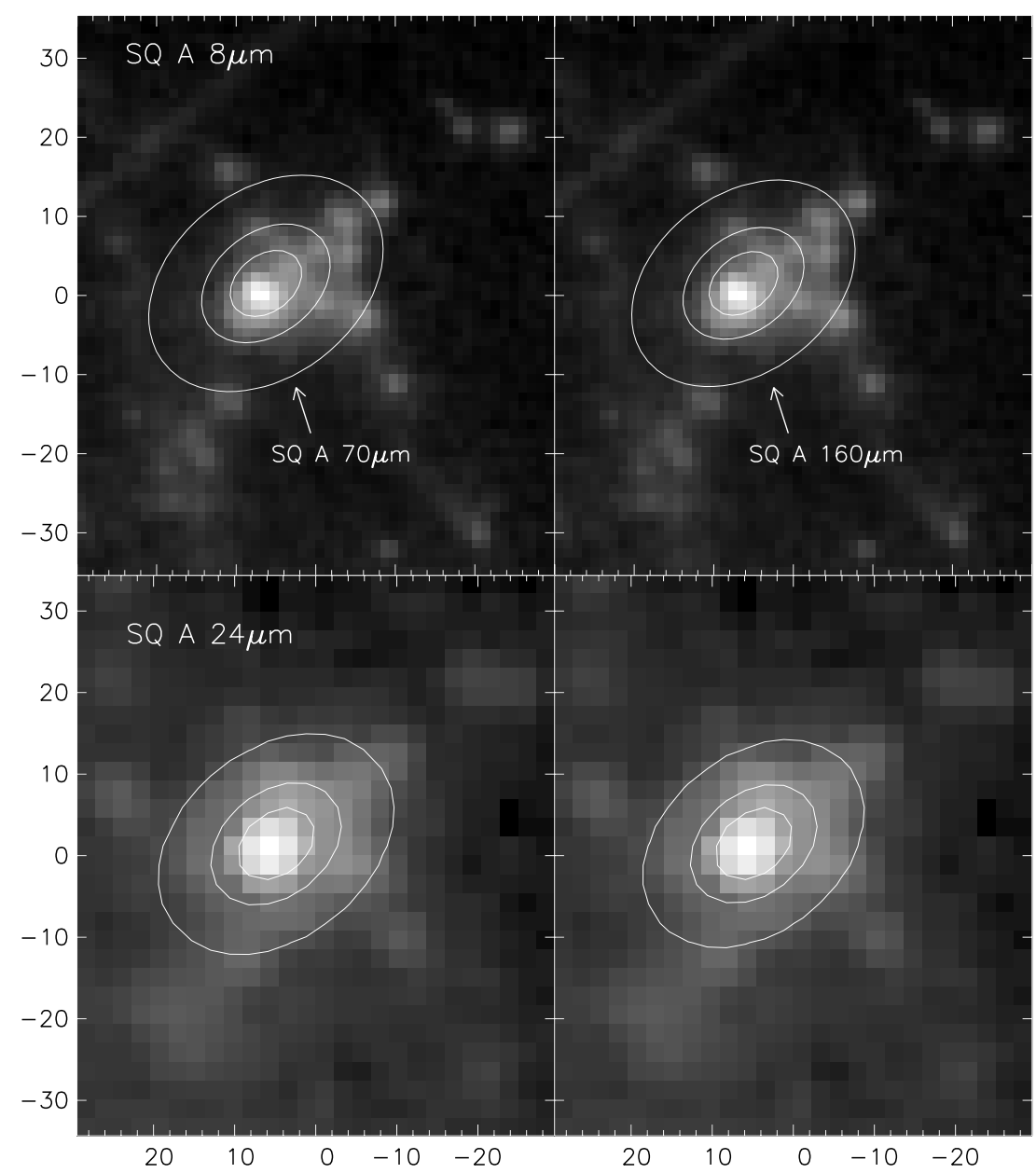

Figure 5. "Deconvolved" $70 \mu \mathrm{m}$ and $160 \mu \mathrm{m}$ SQ A emission contours overlaid on $8 \mu \mathrm{m}$ (upper row) and $24 \mu \mathrm{m}$ (lower row) maps. Units on the axis are arcseconds.

Table 2

Inferred Infrared Fluxes for the Discrete Sources Detected on the Spitzer $70 \mu \mathrm{m}$ Map

\begin{tabular}{lcccc}
\hline \hline \multicolumn{1}{c}{ Source } & $\begin{array}{c}F_{8 \mu \mathrm{m}} \\
(\mathrm{mJy})\end{array}$ & $\begin{array}{c}F_{24 \mu \mathrm{m}} \\
(\mathrm{mJy})\end{array}$ & $\begin{array}{c}F_{70 \mu \mathrm{m}} \\
(\mathrm{mJy})\end{array}$ & $\begin{array}{c}F_{160 \mu \mathrm{m}} \\
(\mathrm{mJy})\end{array}$ \\
\hline SQ A & $7.9 \pm 0.5$ & $11 \pm 2$ & $134 \pm 15$ & $265 \pm 86$ \\
H II-SE & $3.4 \pm 0.4$ & $7.5 \pm 1$ & $61 \pm 9$ & $186(51) \pm 142(40)$ \\
H II-SW & $2.5 \pm 0.1$ & $2.4 \pm 0.35$ & $31 \pm 5$ & ND \\
SQ B & $3.1 \pm 0.15$ & $5.6 \pm 0.8$ & $63 \pm 8$ & $302(120) \pm 34(13)$ \\
NGC 7319 & $68 \pm 3$ & $185 \pm 8$ & $666 \pm 67$ & $1192 \pm 130$ \\
NGC 7320 & $45 \pm 3$ & $38 \pm 2$ & $827 \pm 85$ & $1899 \pm 196$ \\
H II-N & $0.69 \pm 0.07$ & $1.1 \pm 0.2$ & $52 \pm 6$ & $98(78) \pm 41(33)$ \\
Shock region & $8.1 \pm 0.8$ & $6 \pm 1.5$ & $80 \pm 30$ & $506 \pm 200$ \\
Extended emission & $29 \pm 2$ & $40.6 \pm 5$ & $233 \pm 30$ & $805 \pm 200$ \\
\hline
\end{tabular}

Notes. The $8 \mu \mathrm{m}$ and $24 \mu \mathrm{m}$ fluxes are obtained from aperture photometry while the $70 \mu \mathrm{m}$ and $160 \mu \mathrm{m}$ fluxes for the galaxies and compact star formation regions are the results of the FIR map-fitting technique. The FIR fluxes from the shock region are obtained by the fitting of the FIR residual maps. The MIR and FIR values for the extended emission are derived from the curves of growth of the MIR and FIR maps (see Section 4). The values in brackets are the $160 \mu \mathrm{m}$ fluxes within the size of the $70 \mu \mathrm{m}$ emission.

\subsection{Star Formation Regions and Galaxies}

The FIR map-fitting technique, described previously, allows a precise measure of the flux coming from the compact star formation regions, the AGN galaxy NGC 7319 and the foreground galaxy NGC 7320, all modeled well by convolved elliptical Gaussians (although some sources are not detected at $160 \mu \mathrm{m}$ ). This technique allowed us not only to obtain the total source fluxes but also to derive accurately the source extent. In
Figure 5, as an example, we show the contours of the "deconvolved" $70 \mu \mathrm{m}$ and $160 \mu \mathrm{m}$ emission at the position of SQ A overlaid on the $8 \mu \mathrm{m}$ and $24 \mu \mathrm{m}$ maps. The FIR emission has an MIR counterpart that peaks in the areas where FIR is higher. This is generally true for all the fitted FIR sources and it validates the use of apertures for the photometry of the compact sources at MIR 8 and $24 \mu \mathrm{m}$ whose sizes are derived by the FIR fitting technique. Specifically, at 8 and $24 \mu \mathrm{m}$ we used elliptical apertures having the same axial ratio and orientation as the $70 \mu \mathrm{m}$ 


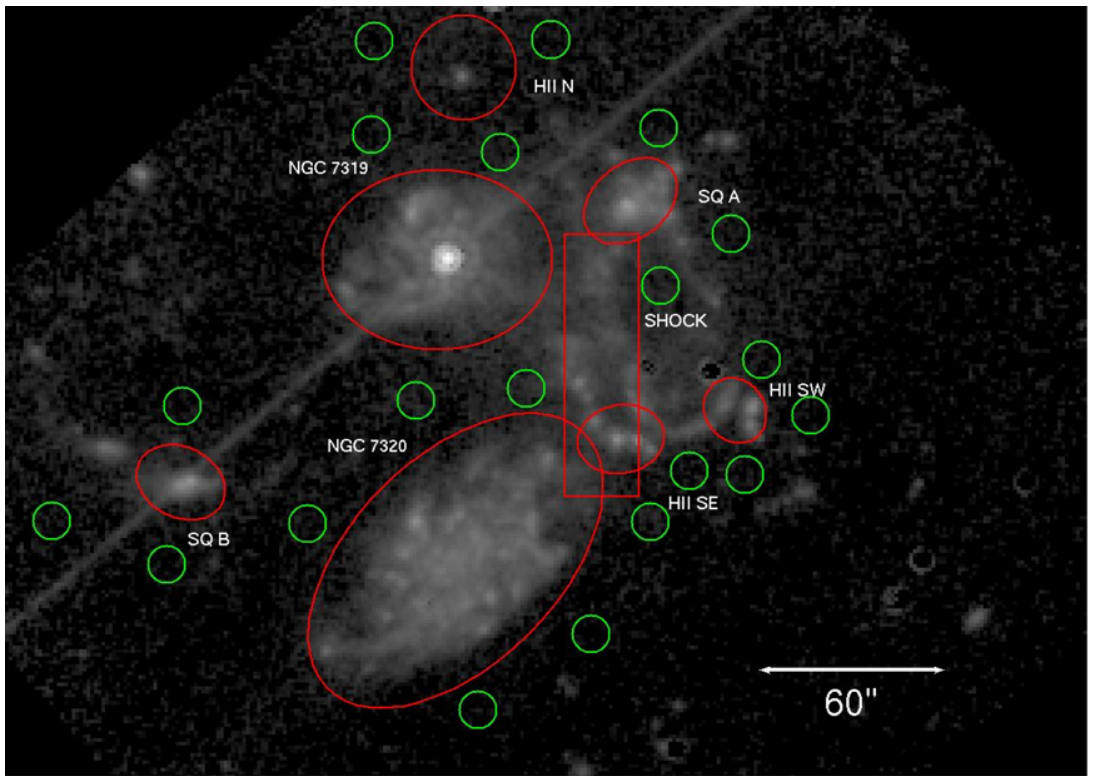

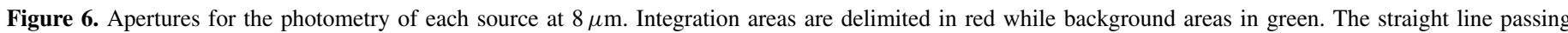
through the AGN galaxy and SQB is a map artifact.

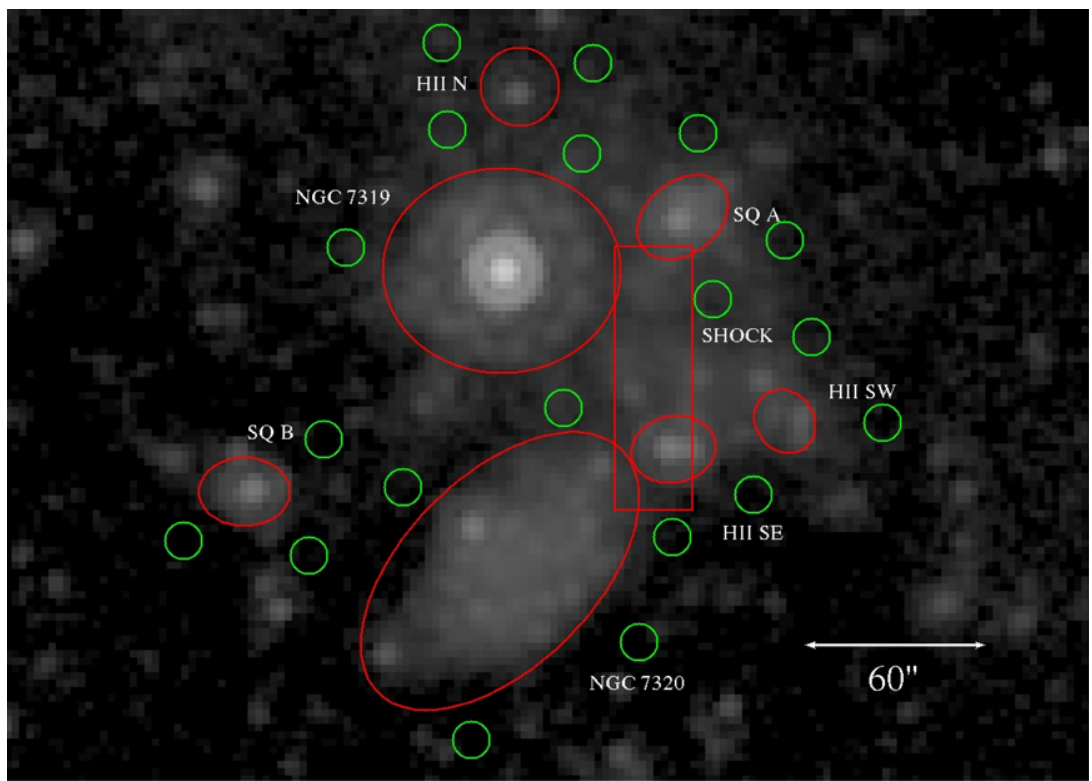

Figure 7. Apertures for the photometry of each source at $24 \mu \mathrm{m}$. Integration areas are delimited in red while background areas in green.

best-fit elliptical Gaussian axis and semi-axis lengths equal to $2.17 \sigma_{70 \mu \mathrm{m}}$ (this area includes $90 \%$ of an elliptical Gaussian total flux). We took sizes based on the $70 \mu \mathrm{m}$ map fit because all the compact sources are clearly seen on that map. These apertures are depicted in red in Figures 6 and 7. The local background for the aperture photometry has been estimated on regions located nearby the central source, where other peaks of emission are not clearly seen (see green circles on Figures 6 and 7). In this way, we are confident that the background level has not been overestimated due to contamination by surrounding sources. We did not apply aperture correction for the photometry at $8 \mu \mathrm{m}$ because this is close to unity for the chosen apertures (typical aperture size about 10 pixels), as reported by the IRAC Data Handbook. At $24 \mu \mathrm{m}$, the chosen apertures typically delimit the first outer ring of the PSF. In this case, we applied an aperture correction equal to 1.16 (MIPS Data Handbook). For the MIR photometry of the galaxies NGC 7319 and NGC 7320, we used large apertures covering most of the emission from these objects and we did not apply aperture corrections because of the large integration area.

The uncertainties on the MIR photometry are given by the quadratic sum of the following contributions: (1) error on the aperture correction, (2) flux calibration uncertainty, and (3) background fluctuations. Since we used elliptical apertures, instead of typical circular apertures, we rather conservatively assumed that the relative error on the aperture correction is $10 \%$ (note that this error is applied only to aperture photometry at $24 \mu \mathrm{m})$. The flux calibration relative uncertainty is equal to $4 \%$ (IRAC and MIPS Handbook) while the error introduced by background fluctuations is derived from the variance of the background mean values in the several areas we selected nearby the sources. The measured fluxes and 


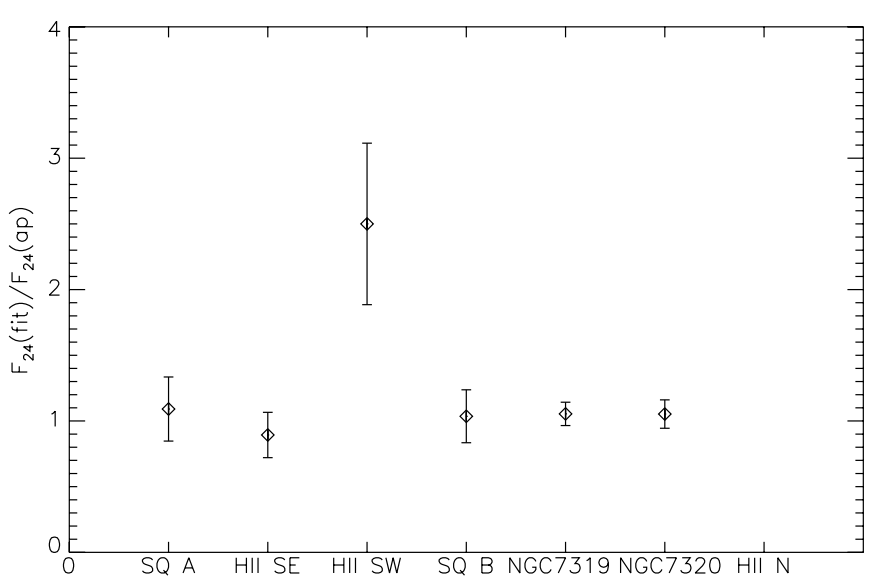

Figure 8. Ratio between fluxes inferred from the fitting technique and from aperture photometry for the sources on the $24 \mu \mathrm{m}$ map.

uncertainties for both MIR and FIR wavelengths are given in Table 2.

Since we are using different methods to derive the source fluxes on the MIR maps and the FIR maps, it is important to check that these two photometric techniques give consistent results. To verify this, we performed the source fitting technique on convolved MIR maps and derived the source fluxes exactly as we did for the FIR maps. Details of this test are shown in Appendix A. In Figure 8, we plot the ratio of the $24 \mu \mathrm{m}$ fluxes inferred by the fitting technique and by aperture photometry for each source. For all the sources, except for $\mathrm{H}_{\mathrm{II}} \mathrm{SW}$, the difference between the fluxes obtained using different methods is less than $20 \%$. The discrepancy between the fluxes for H IISW arises because of the presence of a compact $24 \mu \mathrm{m}$ source at the nearby nucleus of NGC $7318 \mathrm{a} .{ }^{15}$ This source is included in footprint of the Gaussian returned by the fitting procedure but is outside the integration area used for the aperture photometry.

\subsection{Shock Region}

Figure 9 shows the east-west profiles, along a line passing through the center of the shock ridge, extracted from the FIR residual maps, the X-ray, and the VLA $21 \mathrm{~cm}$ radio continuum maps convolved to the resolution of the $160 \mu \mathrm{m}$ map $\left(\mathrm{FWHM} \approx 40^{\prime \prime}\right)$. From these profiles, one can see that the FIR width is significantly larger than the shock ridge width as seen both in the X-ray and radio. Using the current data, it is impossible to say if this discrepancy is due to confusion with fainter unrelated infrared sources or to a systematic change in the width of the emitting region of the shock between the X-ray and the FIR. Nevertheless we estimated the flux coming from the shock ridge by fitting the FIR residual maps with a simple twocomponent model: a PSF convolved uniform ridge, used to fit the dust emission in the shock region and whose size $\left(20^{\prime \prime} \times 80^{\prime \prime}\right)$ was derived from X-ray data, plus a uniform component. In this procedure, the level of the fitted uniform component is influenced by the fact that the actual FIR source is more extended than the X-ray source. Taking into account the ambiguity in identifying all the flux from the more extended FIR emission with the shock, we have assigned extremely conservative flux uncertainties. Specifically, the upper and lower limits defined by the quoted error bars in Table 2 are the fluxes contributed by the uniform emission component underlying the solid angle

\footnotetext{
15 The presence of a compact X-ray source (see Figure 1) and a compact radio
} source (see Xu et al. 2003) suggests that this source is a low-luminosity AGN.

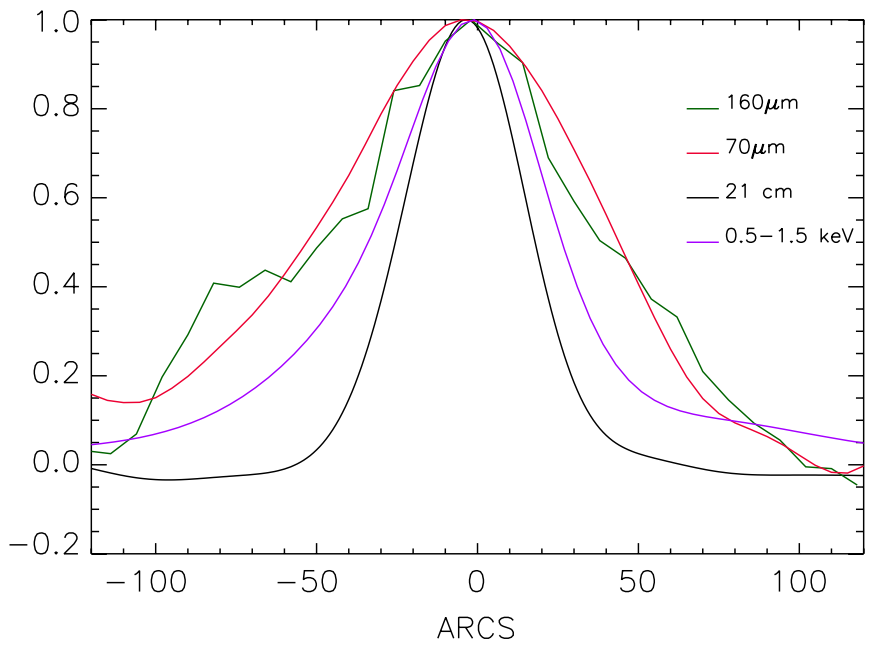

Figure 9. East-west profile of the shock region at several wavelengths. The profiles for the $70 \mu \mathrm{m}, \mathrm{X}$-ray, and radio maps are derived after convolution to the resolution of the $160 \mu \mathrm{m}$ map $\left(\mathrm{FWHM}=40^{\prime \prime}\right)$. The profiles are derived from a horizontal strip passing through the center of the shock region (R.A. $=22^{\mathrm{h}} 35^{\prime} 59.7$, decl. $=+33^{\circ} 58^{\prime} 14^{\prime \prime}$. 2 ) and of vertical width equal to $10^{\prime \prime}$.

of the shock ridge (convolved with the PSF) in the cases the uniform emission component has a brightness equal to twice or zero times the value given by the fit.

To measure the MIR 8 and $24 \mu \mathrm{m}$ emission from the shock region, we simply integrated the emission in the same rectangular area used before to define the shock ridge component in the fit of the FIR residual maps. The adopted apertures are shown in Figures 6 and 7. Before the integration, we masked all the areas inside the apertures we used for the photometry of other sources, because the MIR emission in that areas is mainly connected with the corresponding sources that we fitted and subtracted from the FIR maps before the measure of the shock region flux. For the fraction of the masked regions that falls inside the shock region integration area, we assumed that the surface brightness is equal to the average brightness on the other regions inside the aperture. The background level has been measured on areas around the rectangular aperture where no peaks of emission are clearly seen. The quoted error on the integrated fluxes is the sum of the contributions due to background fluctuation and flux calibration error.

\subsection{Extended FIR Emission}

We have measured the amount of extended flux on the FIR residual maps within a radius of $90^{\prime \prime}$ from the shock center, an area approximately equal to that covered by the X-ray HALO region as defined in Trinchieri et al. (2005). We performed this flux measurement in the following way. First, we constructed radial curves of growth of the integrated emission on the FIR residual maps starting from the shock center. These curves of growth are shown in Figure 10. As one can see, the integrated emission continues to grow somewhat beyond the $90^{\prime \prime}$ radius. However, we considered only the flux within this limit because it can be directly related to the X-ray halo extent. We took the curve of growth values for the integrated fluxes at $90^{\prime \prime}$ and we subtracted the contribution from the shock region, estimated in Section 4.2. Similarly as before, the uncertainties on the fluxes are mainly due to the mutual contamination between shock ridge and extended emission. Therefore, we assumed the same conservative errors that we assigned to the shock region FIR fluxes. For the estimate of the extended MIR emission, 


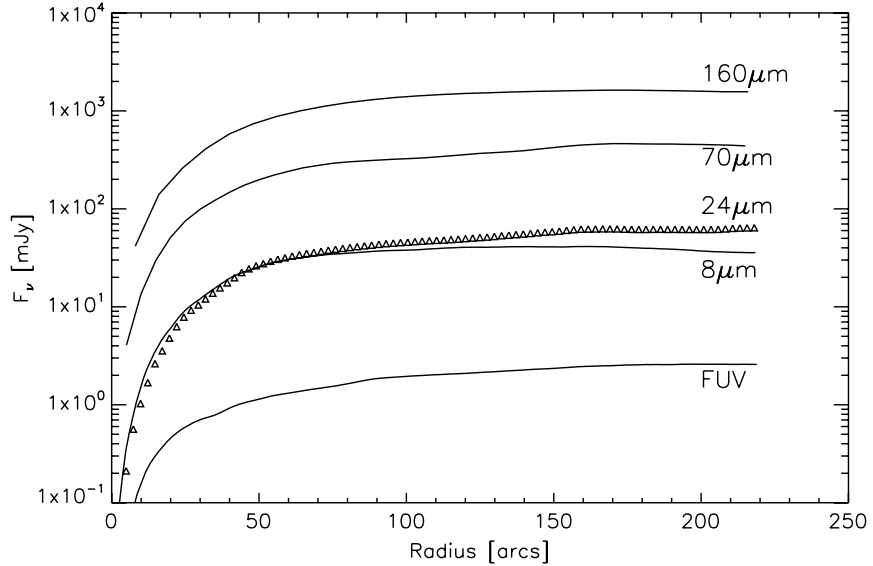

Figure 10. Radial curves of growth of the FUV, MIR, and FIR emission after masking or subtraction of star formation regions and the two galaxies NGC 7320 and NGC 7319 (see Section 4.3). The zero on the $X$-axis corresponds to the center of the shock region: R.A. $=22^{\mathrm{h}} 35^{\prime} 59.7$, decl. $=+33^{\circ} 58^{\prime} 14^{\prime \prime} .2$.

we have used an analogous method. We constructed radial curves of growth, starting from the shock region center, after having masked all the compact sources and galaxies whose photometry has been described in Section 4.1 (note that the shock region is not masked). For the calculation of the MIR curves of growth, we took into account the missing areas, those that we masked, assuming that their brightness is equal to the average brightness inside the circular annuli passing through them. The derived curves are shown in Figure 10. Exactly as for the FIR measurements, we took the value of the curve of growth at $90^{\prime \prime}$ and subtracted the emission from the shock region in order to obtain the integrated emission from the extended area corresponding to the X-ray halo. The flux uncertainties, in this case, are derived summing quadratically background fluctuation, calibration errors, and the error on the shock ridge flux.

\section{MODELING THE INFRARED SEDs}

The spectral energy distribution (SED) of dust emission is determined by the heating mechanism, the intensity and color of the radiation fields (for the case of photon heating), the temperature and density of the hot plasma (for the case of collisional heating), and by the amount, size distribution, and chemical composition of the emitting grains. Ideally, one would solve for the distribution of photon sources, hot plasma, and grains using a self-consistent radiation transfer analysis to fit the entire X-ray/UV/optical-MIR/FIR SED for each source, analogous to the treatment of photon-heated dust in disk galaxies and starburst galaxies (see Popescu \& Tuffs 2010 for a recent review). However, due to the extra dimension of collisional heating and the unusual geometry of the optical/UV and infrared emission from SQ, this approach would require a very individualized treatment which is well beyond the scope of this paper. We therefore adopt here a hybrid approach fitting just the MIR/FIR SED with superpositions of dust emission templates where each template is appropriate for specific dust emission regions: $\mathrm{H}$ II/photodissociation regions (PDRs), diffuse-photonpowered dust emission, AGN torus emission, and collisionally heated dust embedded in hot X-ray plasma. Each of these templates is calculated self-consistently in terms of physical input parameters, as described in detail in Appendix B. In this approach, the amplitude of the $\mathrm{H}_{\mathrm{II}} / \mathrm{PDR}$ template, derived from the model of Dopita et al. (2005) and Groves et al. (2008) and shown in Figure 11 (left panel), quantifies the obscured component of ongoing star formation, whereas the amplitude of the diffuse dust emission template (shown in Figure 12) quantifies dust emission powered by longer range photons to which older stellar populations can also contribute. In the latter case, a family of SEDs is calculated according to the strength and color of the radiation field which are both expected to vary according to radiation transfer effects and the relative contribution of young and old stellar populations. A family of SEDs is also calculated for the collisionally heated dust emission template (Figure 13) corresponding to a range of plasma parameters and to different grain size distributions thus accounting for the expected effect of the plasma on the grain size distribution. For both the photon-heated and collisionally heated dust emission templates, the stochastic emission from impulsively heated grains, which is important to determine the MIR emission, is calculated. In the case of the AGN template SED, we have used the existing self-consistent model of Fritz et al. (2006; the right panel of Figure 11).

The fits were done by minimizing $\chi^{2}$ for each source, defined as

$$
\chi^{2}=\sum\left(\frac{F_{\nu i}^{\text {model }}-F_{i}^{\text {obs }}}{\sigma_{i}}\right)^{2}
$$

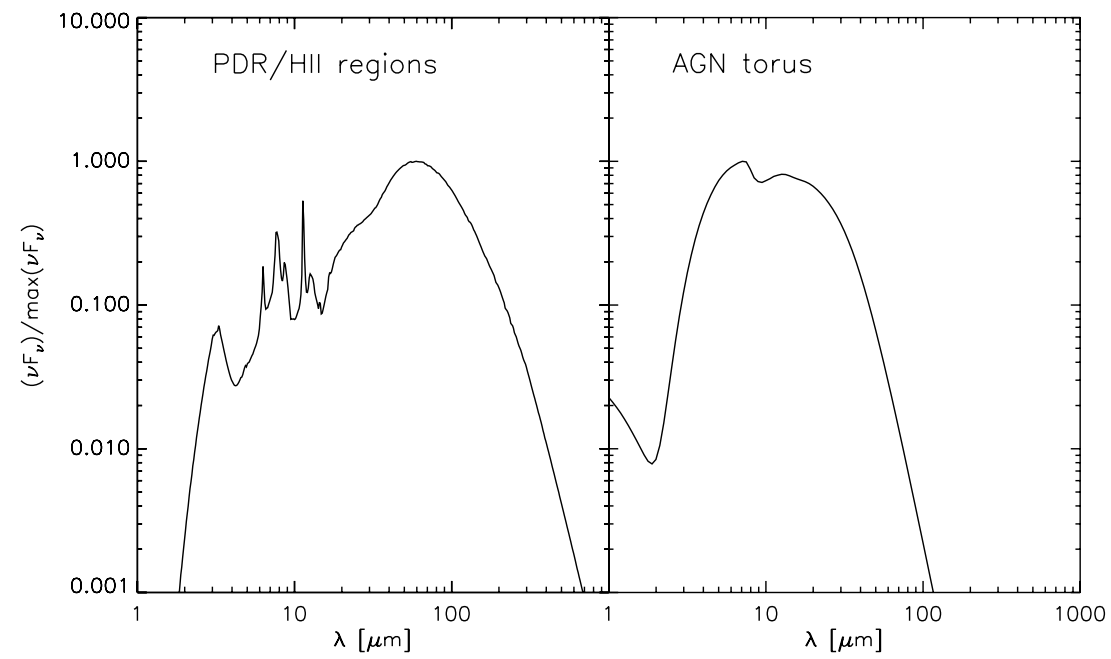

Figure 11. PDR/H II region dust emission template (left panel) and AGN torus dust emission template (right panel). 


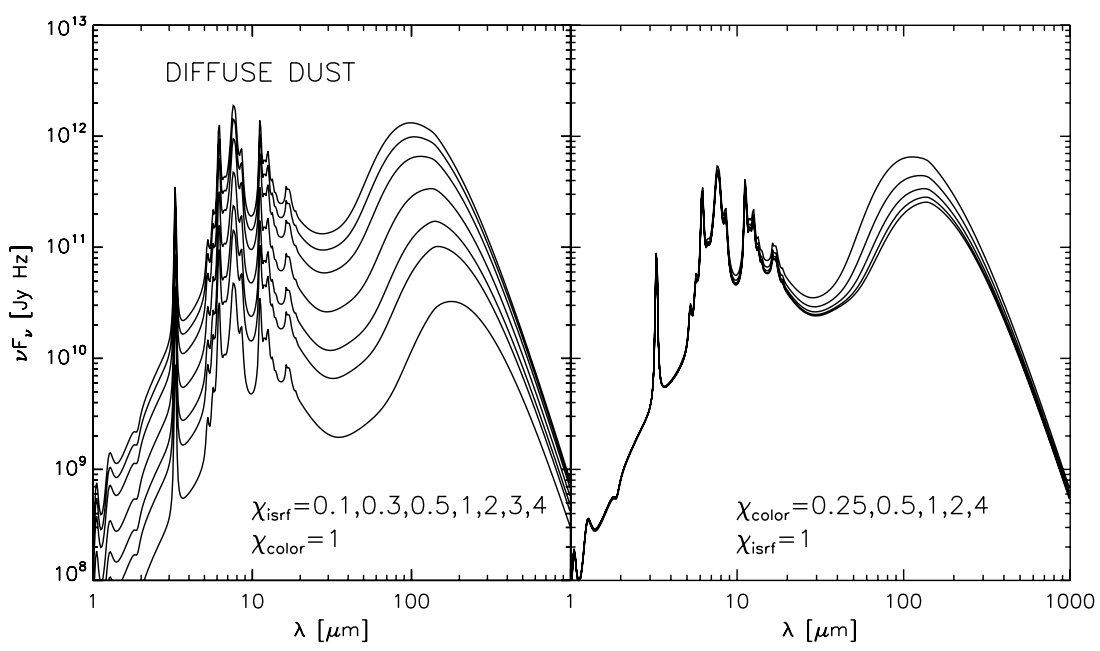

Figure 12. Diffuse-photon-heated dust SEDs for different choices of $\chi_{\text {isrf }}$ and $\chi_{\text {color }}$. In the left panel, the SEDs are calculated assuming $\chi_{\text {color }}=1$ and several values of $\chi_{\text {isrf }}$ (the higher curves correspond to higher values of $\chi_{\text {isrf }}$ ). In the right panel, we assumed $\chi_{\text {isrf }}=1$ and different values of $\chi_{\text {color }}$ (similarly, the higher curves correspond to higher values of $\chi_{\text {color }}$ ). The SEDs are calculated assuming a distance equal to $94 \mathrm{Mpc}$ (SQ distance) and a dust mass $M_{\mathrm{d}}=10^{7} M_{\odot}$.

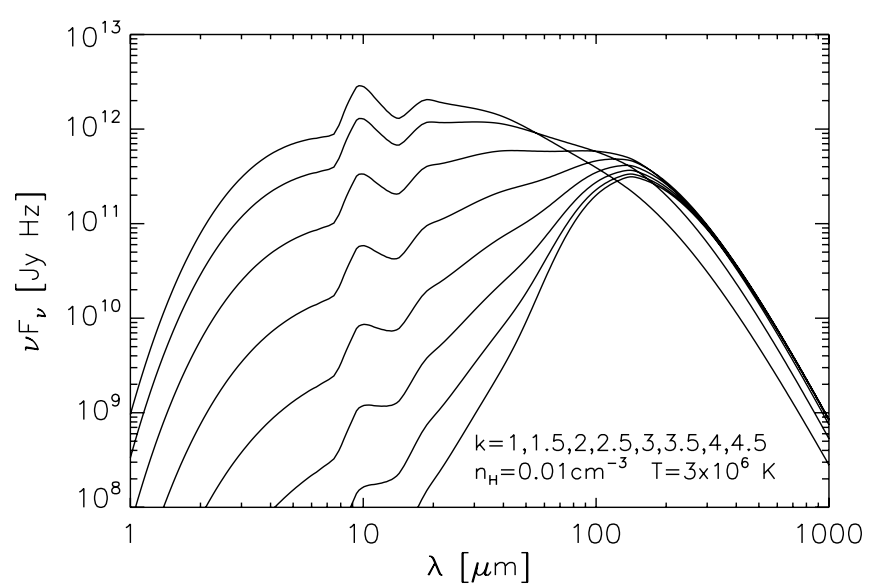

Figure 13. Theoretical collisionally heated dust SEDs for several values of the size distribution exponent $k$. From the lowest to the highest curve, the $k$ values are, respectively, 1, 1.5, 2, 2.5, 3, 3.5, 4, and 4.5. The SEDs are calculated assuming for the plasma density and temperature, respectively, $n_{\mathrm{H}}=0.01 \mathrm{~cm}^{-3}$ and $T=3 \times 10^{6} \mathrm{~K}$, a dust mass $M_{\mathrm{d}}=10^{7} M_{\odot}$ and a distance equal to $94 \mathrm{Mpc}$.

where $F_{i}^{\text {obs }}$ and $\sigma_{i}$, respectively, are the observed flux densities and their associated uncertainties, and $F_{v}^{\text {model }}$ is the corresponding model prediction which is related to a given theoretical spectra $F_{v}$ through $F_{v}^{\text {model }}=K F_{v}$. The color correction $K$ for each Spitzer band is calculated according to the formulae provided by the IRAC and MIPS data handbooks (see Appendix D). It is particularly important for the $8 \mu \mathrm{m}$ band where the emission spectra vary quickly along the bandwidth because of PAH line emission. The color correction can be up to $80 \%$ at $8 \mu \mathrm{m}$, while it is generally less than $10 \%$ for the other bands.

\subsection{Star Formation Regions}

In this section, we describe the SED fits for the star formation regions SQ A, H II $^{-S E}, \mathrm{H}_{\mathrm{II}}-\mathrm{SW}$, and SQ B. We fitted all the observed SEDs as the superposition of two components. The first component is the $\mathrm{PDR} / \mathrm{H}$ II region dust emission template that helps to fit the emission from warm dust located very close to young stars. The second component is the diffuse-photonheated dust emission template that fits the emission from diffuse dust near star formation regions. This diffuse dust is heated by a combination of UV photons escaping from the PDR/H II region and any ambient large scale UV/optical radiation field pervading the region. We performed the fit varying four free parameters: the amplitude of the $\mathrm{H}$ II region/PDR SED template (determined by the parameter $F_{24}^{\mathrm{HII}}$, the fraction of $24 \mu \mathrm{m}$ flux contributed by this component); the diffuse dust mass $M_{\text {dust }}$; and the strength and the color of the diffuse radiation field, determined by two parameters, $\chi_{\text {isrf }}$ and $\chi_{\text {color }}$ (see Appendix B for details). As one can notice, there are no degrees of freedom in these fits. Therefore, it is not possible to estimate the goodness of the fit (in the sense of the fidelity of the model) from a $\chi^{2}$ test. Nonetheless we estimated the error on the best-fit dust masses and total dust luminosities from a multidimensional analysis of $\chi^{2}$ near to its minimum. The uncertainty on the dust mass is the minimum dust mass variation that gives $\Delta \chi^{2}=\chi^{2}-\chi_{\min }^{2}$ values always greater than one, independently from the values of all the other parameters. The total luminosity error bar is determined by the lowest and highest values of the total luminosity in the subspace of fitting parameters determined by $\Delta \chi^{2} \leqslant 1$.

The SED fits are shown in Figure 14 (note that for $\mathrm{H}_{\text {II- }}$ SE and SQ B we used the $160 \mu \mathrm{m}$ fluxes within the $70 \mu \mathrm{m}$ source size to limit contamination from neighbors). Table 3 shows the best-fit parameters, together with the estimate of the uncertainty on the dust mass, the total infrared dust luminosity and the contribution to this total infrared luminosity from optical heating of diffuse dust by the diffuse radiation field, UV heating of diffuse dust by the diffuse radiation field and localized UV heating of dust in PDR/H II regions. The predicted radiation fields needed to account for diffuse emission component are at least as strong as the local ISRF in the Milky Way with a strong variation in color. From the plots, one can see that the $24 \mu \mathrm{m}$ emission is dominated by $\mathrm{H}$ II region/PDR emission, as expected, while $8 \mu \mathrm{m}$ and FIR points are generally dominated by diffuse emission, in accordance with fully self-consistent radiative transfer models of galaxies (Popescu et al. 2010). This is consistent with a picture where MIR emission is produced by regions very close to young stars whilst FIR emission and PAHs emission come from dust illuminated by dilute radiation fields, as observed in galaxy disks (e.g., Bendo et al. 2008)

\subsection{AGN Galaxy NGC 7319}

The observed SED of the Seyfert 2 galaxy NGC 7319 differs markedly from the SEDs we obtained for the other sources in SQ, for which the power emitted at $24 \mu \mathrm{m}$ is typically 


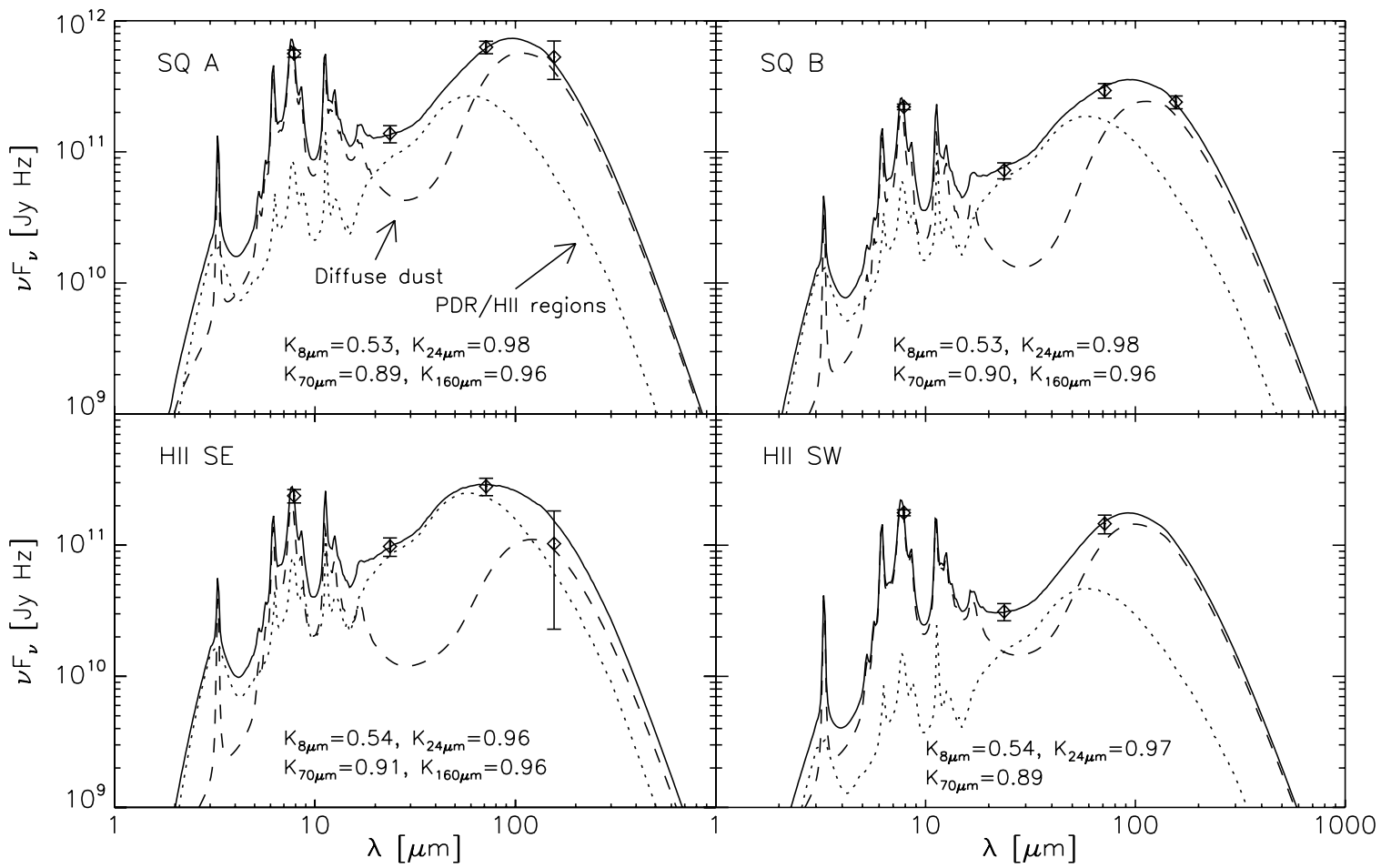

Figure 14. SEDs of discrete SQ star formation regions: the plotted curves are the best-fit SED (solid line), the contribution from PDR/H II regions (dotted curve), and diffuse dust (dashed line). The values $K_{8 \mu \mathrm{m}}, K_{24 \mu \mathrm{m}}, K_{70 \mu \mathrm{m}}$, and $K_{160 \mu \mathrm{m}}$, shown in each plot of this and the following figures, are the color corrections applied to each fitted point.

Table 3

Results from the Infrared SED Fitting

\begin{tabular}{|c|c|c|c|c|c|c|c|c|}
\hline Source & $\begin{array}{c}M_{\text {dust }} \\
\left(10^{7} M_{\odot}\right) \\
\end{array}$ & $\chi_{\text {isrf }}$ & $\chi_{\text {color }}$ & $F_{24}$ & $\begin{array}{c}L_{\mathrm{UV}}^{\text {abs,local }} \\
\left(10^{43} \mathrm{erg} \mathrm{s}^{-1}\right) \\
\end{array}$ & $\begin{array}{c}L_{\mathrm{opt}}^{\text {abs, diffuse }} \\
\left(10^{43} \mathrm{erg} \mathrm{s}^{-1}\right) \\
\end{array}$ & $\begin{array}{c}L_{\mathrm{UV}}^{\text {abs,diffuse }} \\
\left(10^{43} \mathrm{erg} \mathrm{s}^{-1}\right) \\
\end{array}$ & $\begin{array}{c}L_{\text {tot }} \\
\left(10^{43} \mathrm{erg} \mathrm{s}^{-1}\right) \\
\end{array}$ \\
\hline SQ A & $0.6_{-0.2}^{+0.1}$ & 2 & 2 & 0.65 (H II) & 0.45 & 0.61 & 0.36 & $1.4_{-0.1}^{+0.2}$ \\
\hline H II-SE 7318b & $0.22_{-0.05}^{+0.04}$ & 2 & 0.25 & $0.86(\mathrm{H} \mathrm{II})$ & 0.42 & 0.10 & 0.12 & $0.64_{-0.06}^{+0.06}$ \\
\hline H II-SW 7318b & $0.11_{-0.01}^{+0.01}$ & 4 & 1 & $0.50(\mathrm{HII})$ & 0.08 & 0.14 & 0.12 & $0.34_{-0.01}^{+0.03}$ \\
\hline SQ B & $0.37_{-0.04}^{+0.09}$ & 1 & 4 & $0.80(\mathrm{H} \mathrm{II})$ & 0.31 & 0.28 & 0.10 & $0.70_{-0.03}^{+0.02}$ \\
\hline NGC 7319 & $2.7_{-0.4}^{+0.4}$ & 2 & 4 & $0.90(\mathrm{AGN})$ & 4.13 & 4.01 & 1.47 & $9.6_{-0.3}^{+0.3}$ \\
\hline NGC 7320 & $0.08_{-0.01}^{+0.02}$ & 1 & 4 & 0.45 (H II) & 0.014 & 0.059 & 0.021 & $0.094_{-0.002}^{+0.005}$ \\
\hline Shock region & $4.4_{-1}^{+0.5}$ & 0.3 & 4 & $0.35(\mathrm{H} \mathrm{II})$ & 0.14 & 1.00 & 0.37 & $1.5_{-0.3}^{+0.1}$ \\
\hline Extended emission & $47_{-4}^{+3}$ & 0.1 & 1 & $0.71(\mathrm{H} \mathrm{II})$ & 1.71 & 1.56 & 1.30 & $4.6_{-0.5}^{+0.2}$ \\
\hline
\end{tabular}

Notes. Column 1: fitted source; Column 2: cold component dust mass; Columns. 3 and 4: radiation field $\chi_{\text {isrf }}$ and $\chi_{\text {color }}$ parameters (see Section 5); Column 5: fraction of $24 \mu \mathrm{m}$ flux contributed by $\mathrm{H}$ II regions or AGN torus templates; Column 6: luminosity of dust in PDR/H II regions or in AGN torus in the case of NGC 7319; Column 7: luminosity of diffuse dust powered by the absorption of optical photons of the diffuse radiation field; Column 8: luminosity of diffuse dust powered by the absorption of UV photons of the diffuse radiation field; Column 9: total dust luminosity.

a factor 10 or more smaller than that observed at 70 and $160 \mu \mathrm{m}$. For NGC 7319, the amount of energy emitted at MIR wavelengths is comparable to the FIR luminosity. We first tried to fit the SED of this galaxy using the same H II region/PDR plus diffuse emission components as we have done previously for the star formation regions (we did not include a component of synchrotron emission, originating from the AGN, because an extrapolation of its infrared luminosity from the radio measurements of Aoki et al. (1999) gives values which are 6 orders of magnitudes lower than the observed infrared luminosities). The best fit is shown in the left panel of Figure 15. The fit tries to reproduce the high $24 \mu \mathrm{m}$ flux with the $\mathrm{H}$ II region template but, doing so, completely overestimates the $70 \mu \mathrm{m}$ flux. A second attempt has been performed substituting the H II region/PDR template with an AGN torus template (Fritz et al.
2006; see Appendix B). As one can see from the right panel of Figure 15, the observed data are reproduced much better in this case. Of course the limited number of data points does not allow any inference of the physical properties of the dusty torus or provide precise information on the radiation field. However, the fit does provide an indication that the majority of the $24 \mu \mathrm{m}$ emission in this galaxy is powered directly by the AGN powerlaw radiation. The fitted parameters and the total dust luminosity derived from the AGN torus plus diffuse emission fit are shown in Table 3.

\subsection{Shock Region Emission}

The dust emission SED of the shock region contains MIR and FIR emission components which indicate an inhomogeneous structure of the emitting region. As one can see from the middle 


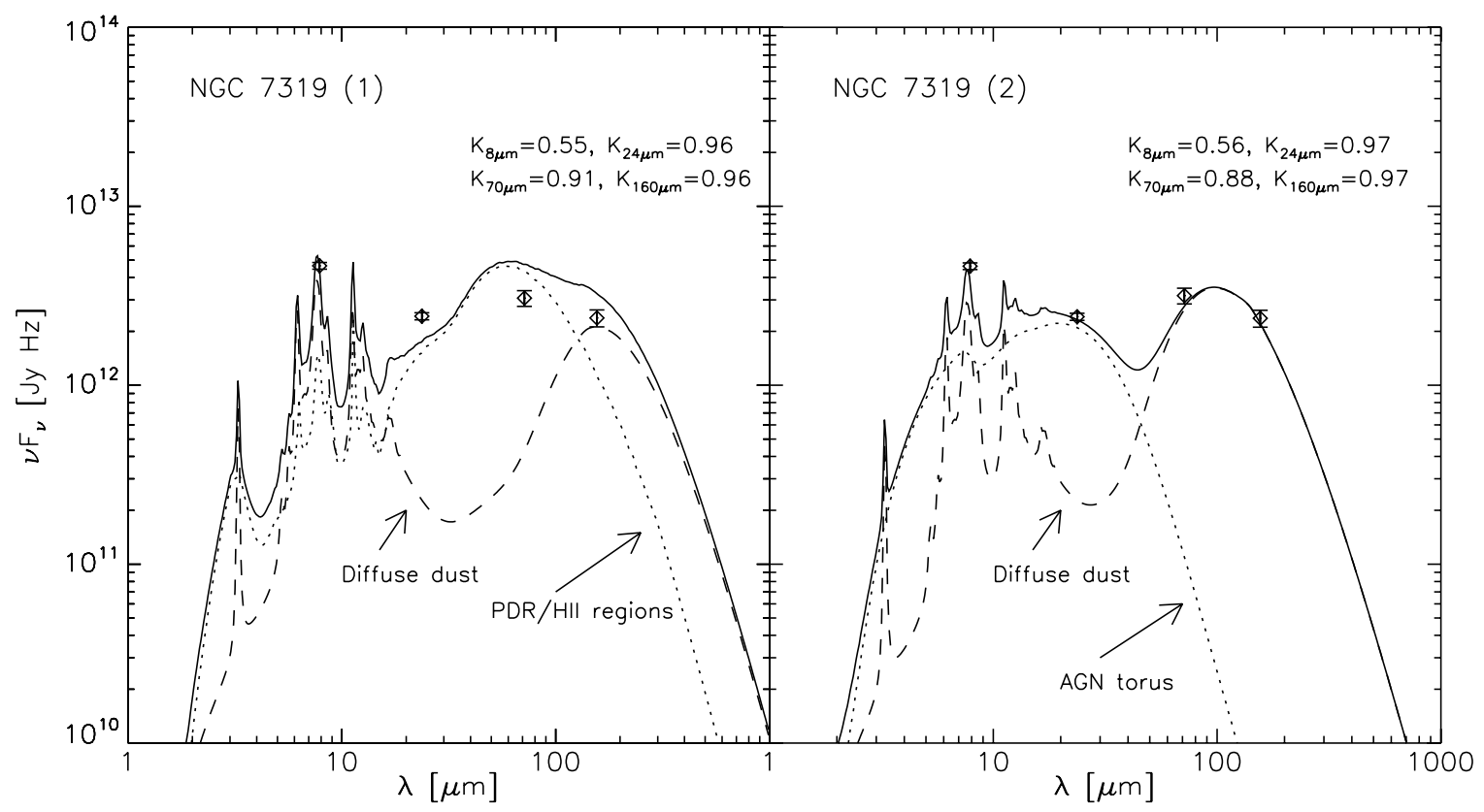

Figure 15. SED of NGC 7319 performed using two different combinations of templates. In the left panel, the best-fit SED (solid line) is calculated using a PDR/H II regions template (dotted curve) and a diffuse dust component (dashed line). In the right panel, the best fit is calculated using an AGN torus dust emission template (dotted) and a diffuse dust component (dashed).

panels of Figure 4, the emission on the FIR residual maps shows a rough correlation with X-ray emission, especially near the center of the shock ridge, suggesting, as mentioned before in Section 3.2, that collisional heating of dust embedded in the hot $\left(T \approx 3 \times 10^{6} \mathrm{~K}\right)$ shocked plasma powers at least some of the observed emission. However, the presence of significant $8 \mu \mathrm{m}$ emission rules out the possibility that the observed dust emission is purely collisionally heated because PAH molecules are not expected to survive in a medium shocked by a wave traveling at more than $125 \mathrm{~km} \mathrm{~s}^{-1}$ (Micelotta et al. 2010). The PAH emission must therefore either be physically unrelated to the shock, coming from another region along the line of sight, or, if associated with the shock, belonging to colder gas phases embedded in the $\mathrm{X}$-ray plasma. The latter scenario would be consistent with the detection of $\mathrm{H}_{2}$ molecular line ${ }^{16}$ (Appleton et al. 2006; Cluver et al. 2010) and $\mathrm{H} \alpha$ (Xu et al. 1999) emission from the shock region. Peculiar ratios between the PAH line features have been detected toward the shock, which suggests an enhanced fraction of neutral and large PAHs compared to typical galaxy environments (Guillard et al. 2010).

The multiphase nature of the gas in the shock region implies that there are at least four potential sources of dust emission in this region: (1) diffuse dust collisionally heated in hot plasma, (2) dust in a colder medium and heated by a diffuse radiation field, (3) H II/PDR dust emission from optically thick clouds with embedded star formation regions, and (4) cold dust emission from optically thick clouds without embedded star formation.

In practice, it is not possible to distinguish model prediction for (2) and (4) over the wavelength range of the currently available data, since the only difference would correspond to a very cold emission component from the interior, self-shielded

\footnotetext{
16 Comparing the surface brightnesses in the $\mathrm{H}_{2} 0-0 S(4)$ and $\mathrm{H}_{2} 0-0 S(5)$ lines, derived from the fluxes given in Table 2 of Cluver et al. (2010; "Main Shock" region), with the $8 \mu \mathrm{m}$ surface brightness derived from the data given in Table 2 of this paper, we estimate the contamination of the Spitzer $8 \mu \mathrm{m}$ band by $\mathrm{H}_{2}$ line emission to $\mathrm{be} \approx 10 \%$.
}

regions of the optically thick clouds, which will only become apparent at longer submillimeter wavelengths. Therefore, we only consider explicitly here components (1)-(3), ignoring component (4). Furthermore, the four available data points are insufficient to simultaneously fit these three potential sources of dust emission, especially the diffuse-photon-heated and collisionally heated components, which are both predicted to peak at FIR wavelengths. Therefore, we decided to perform the SED fit in two different ways corresponding to the two opposite cases, where collisional heating is either responsible for the entire FIR emission or is completely negligible. We followed this approach in order to understand which mechanism is predominant in powering the diffuse dust emission. First, we fitted the shock region SED as a superposition of two components: the $\mathrm{H}$ II region/PDR template, to fit MIR emission possibly associated with star formation regions and a collisionally heated dust SED template (see Appendix B.4). This model is appropriate for the case where photon-heated diffuse FIR emission is negligible compared to the collisionally heated dust emission. Given the plasma physical properties (fixed by the X-ray emission, as described in Appendix C), the free parameters in this fit are the amplitude of the H II region template and the collisionally heated dust mass. We performed these fits for the highest and the lowest densities admitted by the X-ray data, however, the final results are quite similar. In the upper panels of Figure 16, we show the best fit obtained for the highest considered particle density $n=0.016 \mathrm{~cm}^{-3}$ and temperature $T=3 \times 10^{6} \mathrm{~K}$. As one can see, the collisionally heated component (dashed curve) is sufficient to reproduce the observed data except the $8 \mu \mathrm{m}$ point which, as already remarked, is too high to be due to PDR/ $\mathrm{H}$ II regions, requiring a diffuse-photon-heated contribution. The model curve shown (fitted in this case just to the three longer wavelength data points) was calculated assuming a power-law grain size distribution with exponent $k=2.5$, the expected value in the case of equilibrium between dust injection (with a standard $k=3.5$ interstellar distribution; Mathis et al. 1977) and sputtering of grains in hot plasmas (Dwek et al. 1990). The dust mass required to produce the observed FIR flux densities 


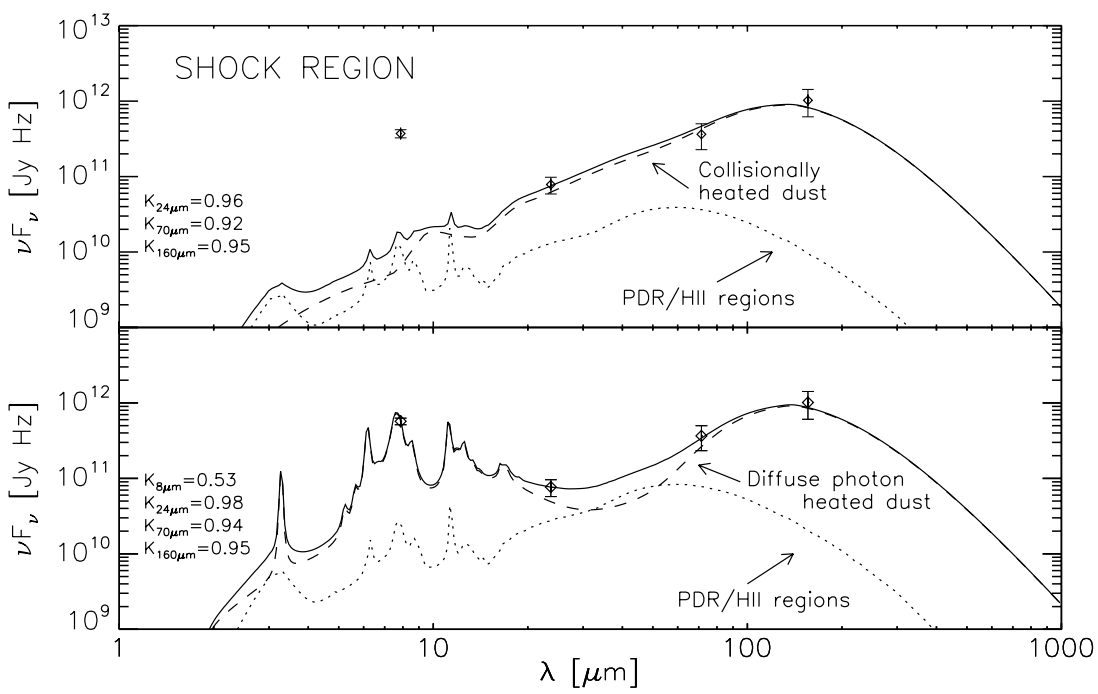

Figure 16. SED of the shock region. The upper panel shows the fit performed using an PDR/H II region template plus a collisional heating component. The hot gas parameters adopted to calculate the collisionally heated dust SED are $n=0.016 \mathrm{~cm}^{-3}$ and $T=3 \times 10^{6} \mathrm{~K}$. The lower panel shows the fit performed using the PDR/H II region template and a uniformly photon-heated dust model.

for the case where collisional heating, rather than diffuse photon heating, is considered is $2 \times 10^{7} M_{\odot}$.

The second fit was performed using the $\mathrm{H}$ II region/PDR template and the photon-heated diffuse dust component. This combination of SED templates represents the case where the entire emission is powered by photons and collisional heating is negligible. Using this combination of SEDs, one can fit all the data points, including the $8 \mu \mathrm{m}$ flux. The best-fit curve is shown in the lower panels of Figure 16. In this fit, the diffuse dust component dominates the emission in all the Spitzer bands, even at $24 \mu \mathrm{m}$, where the contribution of PDR/H II regions is normally predominant. The fitted parameters are given in Table 3. We note that the diffuse radiation fields needed for the fit are colder than those needed to fit the star formation regions in Section 5.1.

In conclusion, the FIR part of the SED from the shock can be fitted by a cold continuum component either from collisional heating, which is cold due to the limited ambient density of plasma particles and the underabundance of small stochastically heated grains, or from photon heating, which is cold due to the low ambient density of photons coupled with a low UV to optical ratio. The only real discriminant favoring photon heating would be the $8 \mu \mathrm{m}$ emission which, as a PAH tracer, cannot be explained by collisional heating. However, it is not entirely clear, on the basis of the current data, that the $8 \mu \mathrm{m}$ emission really does all originate from the shock region.

\subsection{Extended Emission}

In a completely analogous way as for the shock region emission, we tried to model the observed extended emission SED by including a collisionally heated dust emission component. This is motivated by the fact that the FIR residual maps show the presence of extended emission in the area of the brightest component of the X-ray halo emission (the so-called HALO in Trinchieri et al. 2005). In the upper panels of Figure 17, we show the SED fit performed adding two components: H II regions plus collisionally heated dust emission. In this fit, the adopted plasma physical parameters, derived in Appendix C, are $n=0.001 \mathrm{~cm}^{-3}$ and $T=6 \times 10^{6} \mathrm{~K}$ and we again assumed $k=2.5$. Using this combination of SEDs, one can roughly reproduce the SED longward of $24 \mu \mathrm{m}$, but the collisionally heated component dominates only the $160 \mu \mathrm{m}$ flux. The dust mass inferred from this fit (constrained, as in the case for the model fit to the shock SED incorporating collisional heating, only by the three longer wavelength points) is $1.0 \times 10^{8} \mathrm{M}_{\odot}$.

In the lower panel of Figure 17, we show the fit performed adding $\mathrm{H}$ II region and diffuse-photon-heated emission. As before for the shock region, using these two components one can fit the entire spectra including the $8 \mu \mathrm{m}$ point. In this fit, $\mathrm{H}$ II region emission dominates at 24 and $70 \mu \mathrm{m}$ while the diffuse emission is responsible for the 8 and $160 \mu \mathrm{m}$ fluxes. The fitted parameters are shown in Table 3. As for the shock emission, we note that the extended emission component requires a very cold FIR component from the fit even though, observationally speaking, this is a somewhat less robust conclusion, since it really needs to be confirmed with longer wavelength photometry.

\subsection{Foreground Galaxy NGC 7320}

For the SED fit of the foreground galaxy NGC 7320, we used again the usual combination of $\mathrm{H}$ II region plus diffuse-photonheated dust emission components. The best fit is shown in the left panel of Figure 18. The relative contribution of $\mathrm{H}$ II regions to the $24 \mu \mathrm{m}$ flux is reduced compared to the star formation regions, whose SED fits have been shown before. This might be physically understandable since, in this case, we are fitting the emission of a galaxy as a whole, including all the diffuse interstellar medium (ISM) containing small dust particles whose stochastically heated emission can account for a major part of the total MIR emission (e.g., Popescu et al. 2000).

\subsection{Infrared Source North of NGC 7319 (H II-N)}

We attempted to fit the observed emission for this source with a combination of $\mathrm{H}$ II region plus diffuse-photon-heated dust emission. However, this source shows a very peculiar MIR to FIR ratio and, as a consequence, we have not managed to fit the entire spectra. As shown in the right panel of Figure 18, the FIR points are highly underestimated by the bestfit curve. If no systematic errors are present in our measurements, it might be that $\mathrm{HII}-\mathrm{N}$ is a distant background source and the intrinsic colors are heavily redshifted. This possibility 


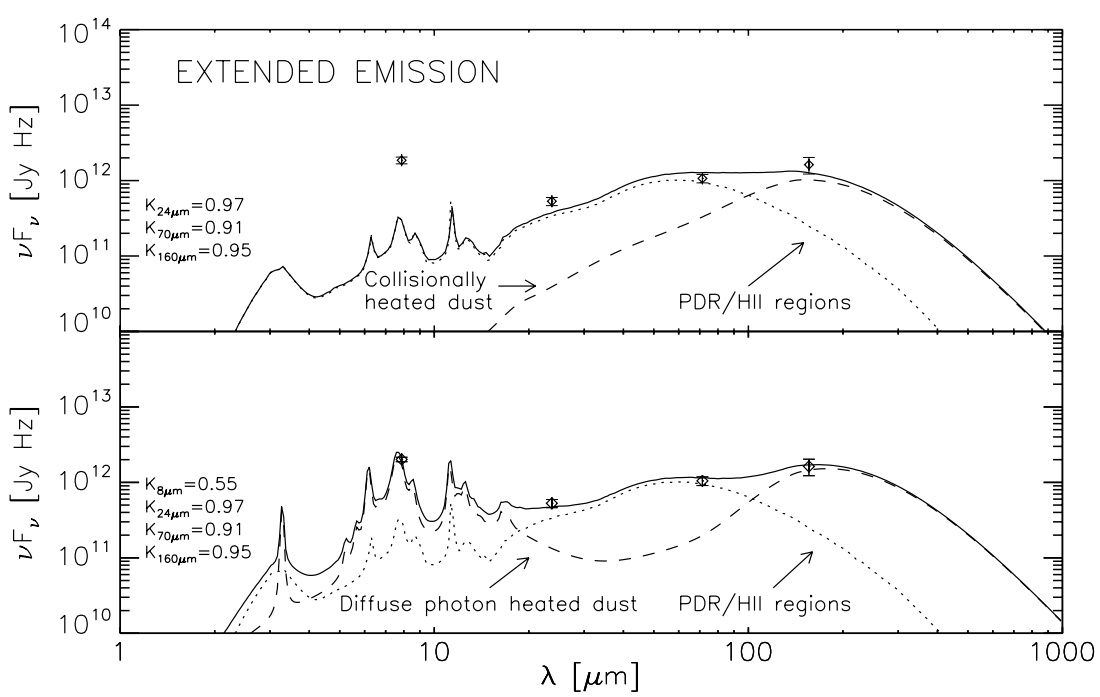

Figure 17. SED of the extended emission. The upper panel shows the fit performed using a PDR/H II region template plus a collisional heating component. The hot gas parameters adopted to calculate the collisionally heated dust SED are $n=0.001 \mathrm{~cm}^{-3}$ and $T=6 \times 10^{6} \mathrm{~K}$. The lower panel shows the fit performed using the $\mathrm{H}$ II region template and a uniformly photon-heated dust model.
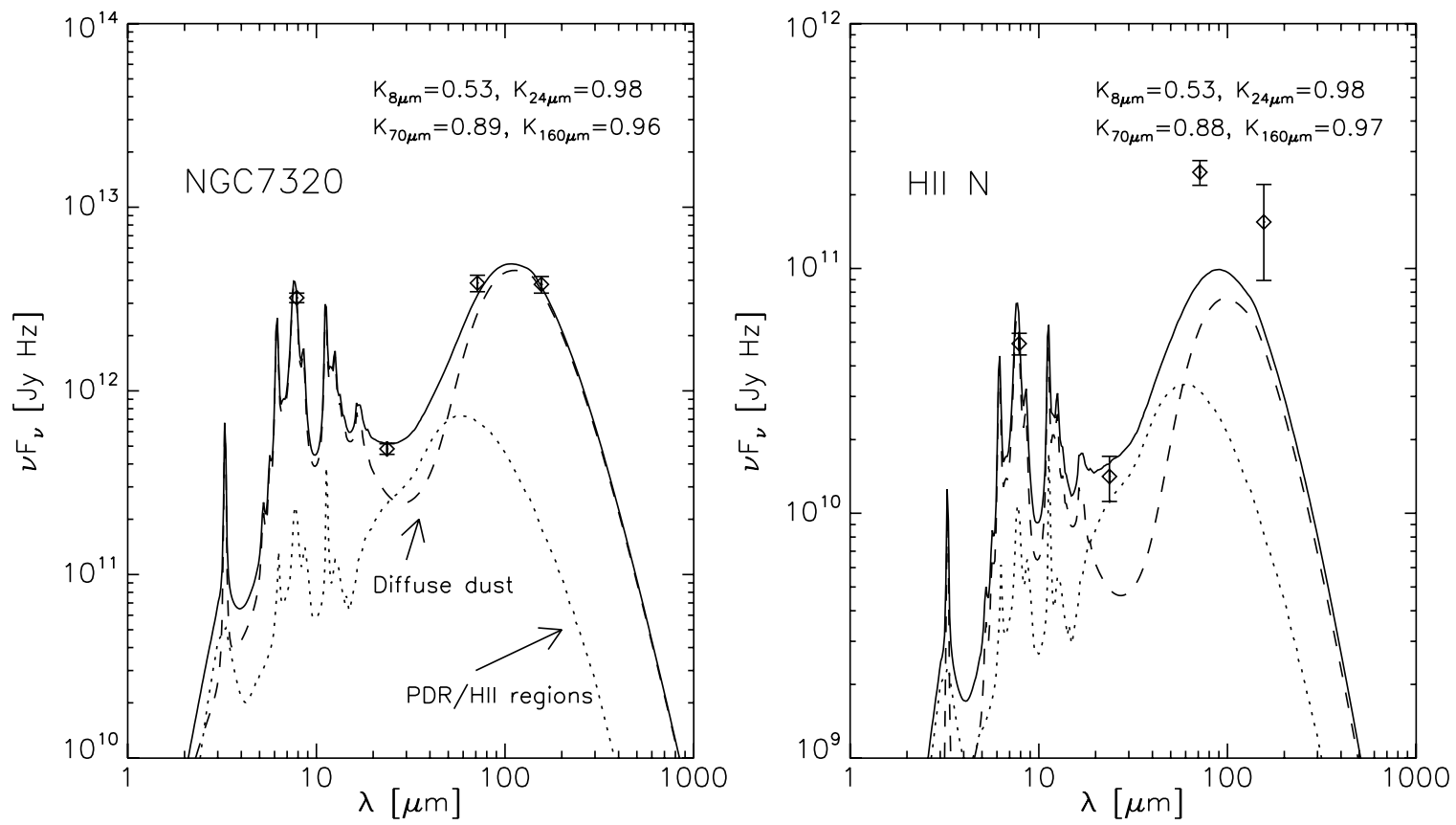

Figure 18. SED of NGC 7320 and H II-N: the plotted curves are the best-fit SED (solid line), the contribution from PDR/H II regions (dotted curve), and diffuse dust (dashed line).

is strongly supported by the presence of a red spiral galaxy at the position of $\mathrm{H}$ II-N, clearly visible on recently released highresolution HST color maps of SQ (Hubble Servicing Mission 4 Early Release Observations, observers: K. Noll et al., available at http://hubblesite.org/newscenter/archive/releases/2009/25/ image $/ \mathrm{x} /$ ).

\section{QUANTIFYING STAR FORMATION RATES AND GAS MASSES IN SQ}

\subsection{Star Formation Rates}

The measurements of UV, recombination line, and dust emission from sources in SQ can in principle be used to derive SFR, provided proper account is taken of the absorption of the $\mathrm{UV} /$ optical photons by dust and subsequent re-emission in the MIR/FIR spectral regimes. Several authors have provided em- pirically based relations achieving this for spiral galaxies on scales of kpc. Calzetti et al. (2007) presented an $\mathrm{H} \alpha$ luminosity based SFR relation: $\operatorname{SFR}\left(M_{\odot} \mathrm{yr}^{-1}\right)=5.3 \times 10^{-42}\left[L\left(\mathrm{H}_{\alpha}\right)_{\text {obs }}+\right.$ $(0.031 \pm 0.006) L(24 \mu \mathrm{m})]$. In this relation, the $24 \mu \mathrm{m}$ luminosity is used to estimate the $\mathrm{H} \alpha$ luminosity obscured by dust. Bigiel et al. (2008) combined GALEX FUV and Spitzer $24 \mu \mathrm{m}$ fluxes to obtain SFR per unit area maps of a sample of spiral galaxies. Like Calzetti et al. (2007), they used a UV-SFR calibration and they used the $24 \mu$ m flux to measure the obscured UV flux.

Simple application of the Calzetti relation to SQ is hampered by the lack of pure measurement of $\mathrm{H} \alpha$ emission driven by star formation. As one can see from Figures 4 and 5 from Sulentic et al. (2001), the shock region $\mathrm{H} \alpha$ emission shows a diffuse component that reflects the north-south ridge seen in the soft Xray regime. This diffuse $\mathrm{H} \alpha$ emission cannot be considered for SFR measurements because, as demonstrated by Xu et al. (2003) 
from spectral line analysis, the $\mathrm{H} \alpha$ emission in the shock region is dominated by shock excitation rather than star formation. In addition, it is not clear that the semiempirical relations derived for spiral galaxies should apply to the sources in SQ.

Therefore, we adopted a new approach, using only observational indicators of star formation activity available for all sources in SQ, and utilizing the results of the fits to the dust emission SEDs given in Section 5. Our method to estimate SFRs is based on a UV-SFR calibration. ${ }^{17}$ Specifically, we adopted the calibration by Salim et al. (2007):

$$
\mathrm{SFR}_{\mathrm{UV}}=1.08 \times 10^{-28} F_{\mathrm{UV}} M_{\odot} \mathrm{yr}^{-1},
$$

where $F_{\mathrm{UV}}$ is the FUV luminosity density in units of erg s $\mathrm{Hz}^{-1}$ which would be observed in the GALEX FUV band in the absence of dust. $F_{\mathrm{UV}}$ can be written as

$$
F_{\mathrm{UV}}=F_{\mathrm{UV}}^{\text {direct }}+F_{\mathrm{UV}}^{\mathrm{abs}, \text { local }}+F_{\mathrm{UV}}^{\mathrm{abs}, \text { diffuse }},
$$

where $F_{\mathrm{UV}}^{\text {direct }}$ is the directly observed unabsorbed component of FUV luminosity density, $F_{\mathrm{UV}}^{\text {abs, local }}$ is the FUV luminosity density locally absorbed by dust in star formation regions, and $F_{\mathrm{UV}}^{\text {abs,diffuse }}$ is the FUV luminosity density absorbed by dust in the diffuse medium surrounding the star formation regions.

We measured $F_{\mathrm{UV}}^{\text {direct }}$ for each source by performing aperture photometry on the GALEX FUV map in a completely analogous way to the photometry we performed on the Spitzer MIR maps, including the construction of the curve of growth after masking the galaxies NGC 7319 and NGC 7320 and the star formation regions (see Figure 10). The final flux densities, shown in Column 4 of Table 4 , include the correction for Galactic foreground extinction $(E(B-V)=0.079$, Schlegel et al. 1998; $A(\mathrm{FUV})=8.24 * E(B-V)$, Wyder et al. 2007). Values for the obscured emission components $F_{\mathrm{UV}}^{\text {abs, local }}$ and $F_{\mathrm{UV}}^{\text {abs, diffuse }}$ were extracted from the fits to the dust emission SEDs by noting that the total infrared luminosity emitted by dust and powered by UV photons can be written as

$$
L_{\mathrm{dust}, \mathrm{UV}}=L_{\mathrm{UV}}^{\mathrm{abs}, \text { local }}+L_{\mathrm{UV}}^{\mathrm{abs} \text {,diffuse }}
$$

where $L_{\mathrm{UV}}^{\text {abs,local }}$ is the luminosity of dust emission in star formation regions, dominated by UV photon heating, and $L_{\mathrm{UV}}^{\mathrm{abs} \text {,diffuse }}$ is the part of the diffuse dust luminosity powered by UV photons, respectively, tabulated in Columns 6 and 8 of Table 3. Since the intrinsic SEDs of the young stellar population are rather flat at UV wavelengths (Kennicutt 1998), it then follows that

$$
F_{\mathrm{UV}}^{\mathrm{abs}, \text { local }}=\frac{L_{\mathrm{UV}}^{\mathrm{abs}, \text { local }}}{\Delta v(\mathrm{UV})}
$$

and

$$
F_{\mathrm{UV}}^{\mathrm{abs}, \text { diffuse }}=\frac{L_{\mathrm{UV}}^{\mathrm{abs} \text { diffuse }}}{\Delta v(\mathrm{UV})},
$$

where $\Delta v \approx 1.8 \times 10^{15} \mathrm{~Hz}$ is the UV frequency width. The total obscured UV luminosity density $F_{\mathrm{UV}}^{\mathrm{abs}}=F_{\mathrm{UV}}^{\mathrm{abs}, \text { local }}+F_{\mathrm{UV}}^{\mathrm{abs} \text {, diffuse }}$ and SFRs are shown in Columns 6 and 7 of Table 4.

As a check of the consistency between our method to derive SFRs and the H $\alpha-24 \mu \mathrm{m}$ SFR relation of Calzetti et al. (2007), we used the latter to derive SFR for the compact star formation

\footnotetext{
17 UV emission traces star formation on timescales of $\sim 100 \mathrm{Myr}$ and therefore, UV derived SFRs could not be appropriate for burst of star formation on smaller timescales.
}

regions in SQ which are the objects closest resembling galaxies. The $\mathrm{H} \alpha$ fluxes have been measured on the interference filter maps published in Xu et al. (1999) while the $24 \mu \mathrm{m}$ fluxes are those derived by aperture photometry (Section 4.1). The results are shown in Columns 2 and 3 of Table 4. As one can see comparing Columns 3 and 7 of that table, the SFR inferred with our method are consistent with the results found using the Calzetti relation (except for $\mathrm{HII-SW}$ but in that case the SED fitting was performed without varying $\chi_{\text {color }}$ because of the non-detection of the source at $160 \mu \mathrm{m})$.

\subsection{Gas Masses}

In the absence of a homogeneous set of observations of gas tracers for the gas phases of interest, namely, $\mathrm{HI}, \mathrm{H}_{2}$, and $\mathrm{X}$-ray-emitting plasma, we proceeded differently for the several sources in determining gas masses. For the star formation regions SQ A, HII-SE, H II-SW, and SQ B, we measured gas mass column densities from the $\mathrm{H}$ I map published by Williams et al. (2002) and the CO maps of Lisenfeld et al. (2002). Specifically, we measured the average atomic and molecular hydrogen column density in the observed areas close to the position of the starburst regions. The inferred gas mass column densities are shown in Columns 9 and 10 of Table 4 (note that for some values only upper limits are available and no $\mathrm{CO}$ observation is available for $\mathrm{H}$ II-SE).

Gas in the shock region is mainly in the form of X-rayemitting plasma and molecular gas (Guillard et al. 2009). From the X-ray luminosities, measured by Trinchieri et al. (2005), we determine the X-ray gas mass $M_{X} \approx 10^{9} M_{\odot}$ (see Appendix C for details). Dividing the total gas mass by the physical area covered by the shock $\left(330 \mathrm{kpc}^{2}\right)$, we obtained the hot gas mass surface density shown in Column 11 of Table 4. To determine the molecular gas mass surface density, we measured the average of several observed positions within the shock area on the $\mathrm{CO}$ maps by Lisenfeld et al. (2002) to obtain $\Sigma\left(\mathrm{H}_{2}\right)=9 M_{\odot} \mathrm{pc}^{-2}$. As one can see from Figure 1 of that paper, most of the observations were performed in the upper part of the shock region. Guillard et al. (2010) and Appleton et al. (2006) found a cold and warm molecular gas surface mass densities in the central parts of the shock region, respectively, equal to $5 M_{\odot} \mathrm{pc}^{-2}$ and $3.2 M_{\odot} \mathrm{pc}^{-2}$. Interestingly enough, the sum of these two values is very close to the cold molecular gas surface density we measured in the upper parts of the shock region.

For the extended emission, the measurement of the corresponding neutral and molecular gas masses cannot be realistically performed because the extended emission cover irregular parts of the large $\mathrm{HI}$ distribution in SQ and there are no CO observations covering the whole group with enough sensitivity to detect a plausible extended molecular hydrogen distribution. The measurement of the corresponding X-ray-emitting gas mass is instead rather straightforward since it can be derived by the $\mathrm{X}$-ray halo luminosity, as for the shock region (see Appendix C). Dividing the total X-ray gas mass by the projected X-ray halo emission area (a circle of radius $\approx 40 \mathrm{kpc}$ ), we determined the hot gas mass surface density shown in Column 11 of Table 4.

\section{DISCUSSION}

If one were to view SQ at a greater distance such that the group would appear as a point-like source to Spitzer (i.e., at a redshift of $\gtrsim 0.5-2$, in the main star-forming epoch of the universe, and also the epoch when galaxy groups were first forming), one 
Table 4

Measured Optical-UV Luminosities, Star Formation Rates, and Gas Amount for the Detected SQ Dust Emitting Sources

\begin{tabular}{|c|c|c|c|c|c|c|c|c|c|c|c|}
\hline Source & $\begin{array}{c}(\mathrm{H} \alpha)^{\mathrm{a}} \\
\left(10^{40} \mathrm{erg} \mathrm{s}^{-1}\right)\end{array}$ & $\begin{array}{c}\mathrm{SFR}_{\mathrm{H} \alpha} \\
\left(M_{\odot} \mathrm{yr}^{-1}\right)\end{array}$ & $\begin{array}{c}F_{\mathrm{UV}}^{\text {direct }} \\
\left(10^{27} \mathrm{erg} \mathrm{s}^{-1} \mathrm{~Hz}^{-1}\right)\end{array}$ & $\begin{array}{c}L_{\text {dust, } \mathrm{UV}} \\
\left(10^{42} \mathrm{erg} \mathrm{s}^{-1}\right)\end{array}$ & $\begin{array}{c}F_{\mathrm{UV}}^{\mathrm{abs}} \\
\left(10^{27} \mathrm{erg} \mathrm{s}^{-1} \mathrm{~Hz}^{-1}\right)\end{array}$ & $\begin{array}{c}\mathrm{SFR}_{\mathrm{UV}} \\
\left(M_{\odot} \mathrm{yr}^{-1}\right)\end{array}$ & 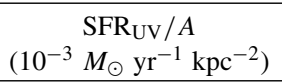 & $\begin{array}{c}(\mathrm{HI}) \\
\left(M_{\odot} \mathrm{pc}^{-2}\right)\end{array}$ & $\begin{array}{c}\Sigma\left(\mathrm{H}_{2}\right) \\
\left(M_{\odot} \mathrm{pc}^{-2}\right)\end{array}$ & $\begin{array}{c}\Sigma\left(\mathrm{H}^{+}\right) \\
\left(M_{\odot} \mathrm{pc}^{-2}\right)\end{array}$ & $Z_{\mathrm{d}}^{\mathrm{b}}$ \\
\hline SQ A & 9. \pm 2 & $0.7 \pm 0.2$ & 2.75 & 8.09 & 4.49 & 0.78 & 6.4 & 7.9 & 6.0 & $\ldots$ & 0.002 \\
\hline H II-SE & $6.6 \pm 1.5$ & $0.5 \pm 0.1$ & 2.32 & 5.44 & 3.02 & 0.58 & 5.8 & $<0.40^{\mathrm{c}}$ & & $\ldots$ & $\ldots$ \\
\hline H II-SW & $3.9 \pm 0.5$ & $0.2 \pm 0.04$ & 2.11 & 1.99 & 1.10 & 0.35 & 5.5 & 3.2 & $<1.7^{\mathrm{c}}$ & $\ldots$ & . \\
\hline SQ B & $1.3 \pm 0.3$ & $0.2 \pm 0.05$ & 0.31 & 4.18 & 2.32 & 0.28 & 2.7 & 5.2 & 4.0 & $\ldots$ & 0.002 \\
\hline NGC 7319 & $\ldots$ & $\ldots$ & 2.01 & $\ldots$ & $\ldots$ & $\gtrsim 0.22$ & $\ldots$ & $\ldots$ & $\ldots$ & $\ldots$ & $\ldots$ \\
\hline Shock & $\ldots$ & $\ldots$ & 6.98 & 5.09 & 2.83 & 1.05 & 3.1 & $\ldots$ & 9.1 & 3.0 & 0.007 \\
\hline Extended & $\ldots$ & $\ldots$ & 22.7 & 30.21 & 16.78 & 4.26 & 1.0 & $\ldots$ & $\ldots$ & 2.4 & 0.01 \\
\hline
\end{tabular}

Notes. Column 1: source name; Column 2: $\mathrm{H} \alpha$ luminosity; Column 3: star formation rate (SFR) derived from the H $\alpha$ and $24 \mu$ m luminosity using the relation given by Calzetti et al. (2007); Column 4 observed GALEX FUV luminosity density corrected for Galactic extinction; Column 5: UV powered infrared dust luminosity; Column 6: absorbed UV luminosity density derived by dividing the UV powered dust luminosity $L_{\text {dust.Uv }}$ by $\Delta v(\mathrm{UV})=1.8 \times 10^{15} \mathrm{~Hz}$; Column 7: SFR based on the inferred total (obscured and unobscured) UV luminosity density, using the relation by Salim et al. (2007); Column 8 : SFRs in Column 7 divided by the projected emission area; Column 9: average neutral hydrogen mass column density; Column 10: average molecular hydrogen mass column density; Column 11: average $\mathrm{X}$-ray-emitting plasma mass column density; Column 12: dust-to-gas ratio $Z_{\mathrm{d}}$ (note that for each source only the corresponding available gas masses are considered for the estimate of $Z_{\mathrm{d}}$ ).

${ }^{a}$ We measured the $\mathrm{H}_{\alpha}$ fluxes from the interference filter maps published in Xu et al. (1999). The quoted flux uncertainties are the quadratic sum of background fluctuations and flux calibration uncertainty $(\approx 10 \%)$.

b The dust-to-gas ratio is equal to $Z_{\mathrm{d}}=M_{\mathrm{d}} /\left(1.4 \times M_{\mathrm{gas}}\right)$ where $M_{\mathrm{d}}$ is the dust mass inferred by the SED fit, $M_{\text {gas }}$ is equal to $N(\mathrm{H}) \times \pi R^{2}$ with $R=\mathrm{FWHM}(\mathrm{rad}) \times$ distance the source size as derived from the $70 \mu \mathrm{m}$ source fit (the factor 1.4 is applied to take into account the contribution from noble gasses).

${ }^{c}$ The value of $\Sigma(\mathrm{HI})$ for $\mathrm{H}$ II-SE is an upper limit because $\mathrm{H}$ I is not detected at that position on the maps by Williams et al. (2002). No value is given for $\Sigma\left(\mathrm{H}_{2}\right)$ because the area was not observed by Lisenfeld et al. (2002). The value of $\Sigma\left(\mathrm{H}_{2}\right)$ for $\mathrm{H}$ II-SW is an upper limit because $\mathrm{CO}$ has not been detected at that position. 


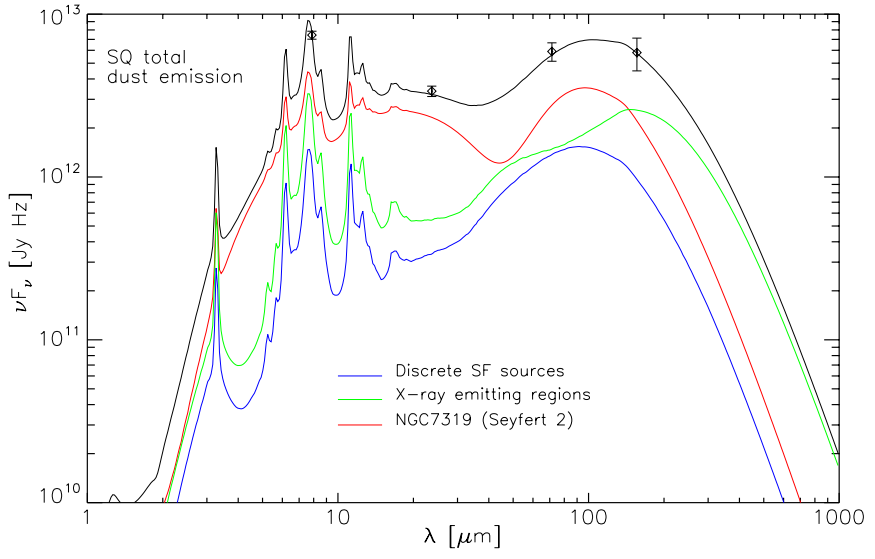

Figure 19. SED of the total SQ dust emission. The plotted points are the sum of the fluxes of all the dust emitting sources in SQ: the discrete sources SQ A, H II-SE, H II-SW, SQ B, the AGN galaxy NGC 7319, the shock region, and the extended emission (the applied color corrections are $0.62(8 \mu \mathrm{m}), 0.97(24 \mu \mathrm{m})$, $0.90(70 \mu \mathrm{m})$, and $0.97(160 \mu \mathrm{m}))$. The plotted curves are the total emission SED (black), the discrete star formation region total SED (blue), the AGN galaxy NGC 7319 SED (red), and the X-ray-emitting region total SED (green). All the SEDs representing the total emission from more sources are derived summing the fitted SEDs as described in Section 5. In the case of the X-ray-emitting region SED, we combined the SED fits for the shock region and the extended emission performed using purely photon-heated dust emission components (see Sections 5.3 and 5.4).

would not regard this as a particularly unusual infrared source. On the basis of the shape of the total dust emission SED, plotted as the black curve in Figure 19, the only noteworthy points would be the quite warm MIR colors and moderately high FIR luminosities which would most likely lead our hypothetical observer to conclude that this source had an AGN, possibly combined with a mild starburst. In this, he would be at least in part correct, as illustrated in Figure 19 by the curves representing the constituent emission components from the AGN galaxy (red), from star formation regions (blue), and the emission from $\mathrm{X}$-ray-emitting regions (green), where one can immediately see the high relative contributions of the AGN galaxy to the $8 \mu \mathrm{m}$ and $24 \mu \mathrm{m}$ emission, respectively, $\sim 50 \%$ and $\sim 70 \%$. In the FIR, although the AGN is still the most luminous individual source, there is emission at a comparable level from the combination of the distributed star formation regions in SQ and the infrared counterparts of the X-ray-emitting shock and halo structures; the combined FIR/submillimeter SED resembles that of starforming galaxies, with an amplitude similar to that of the local starburst galaxy M82.

However, our analysis of the spatially resolved structures has shown that the characteristics of infrared emission in SQ are the very opposite of a nuclear starburst, with the star formation activity enhanced in regions far away from the main bodies of the galaxies. In the following Sections 7.1 and 7.2, we discuss to what extent the nature of this distributed star formation in SQ may differ from the star formation in the disks of individual galaxies, in terms of sources of gas and the star formation efficiency for the group as a whole, and consider the related issue of the extent to which collisional heating of grains in the IGM of SQ may be cooling the IGM and thus contributing to the fueling of the star formation.

\subsection{Star Formation in $S Q$}

The results of the SED fitting to the constituent components of SQ indicate that star formation activity and photons from old stars are the major agents powering the observed global dust
Table 5

Galaxy Optical Photometry and Stellar Masses

\begin{tabular}{lccc}
\hline \multicolumn{1}{c}{ Source } & $g$ & $r$ & $M_{*}{ }^{\mathrm{a}}$ \\
\hline NGC 7319 & 13.58 & 12.83 & 1.6 \\
NGC 7318a & 15.00 & 14.06 & 0.8 \\
NGC 7318b & 13.31 & 12.55 & 2.1 \\
NGC 7317 & 14.58 & 13.78 & 0.8
\end{tabular}

Notes. Column 1: source name; Columns 2 and 3: SDSS $g$ and $r$ apparent magnitudes; Column 4: galaxy stellar mass $\left(10^{11} M_{\odot}\right)$

a The stellar mass has been estimated using one of the relations given by Bell et al. (2003) to calculate the stellar mass to luminosity ratio from SDSS magnitudes: $\log _{10}\left(M / L_{g}\right)=$ $-0.499+1.519 \times(g-r)$ (note that $M / L_{g}$ is in solar units).

emission from the group, supplemented by photons produced by the accretion flows in the AGN and possibly, in the case of the X-ray-emitting regions, by collisional heating. In our quantitative discussion of star formation activity in SQ, we will adopt an initial working hypothesis that the collisional heating mechanism is minor compared with photon heating in the $\mathrm{X}$ ray sources. We will scrutinized this hypothesis in detail in Section 7.2, where we discuss physical constraints of the fraction of the infrared emission that can be collisionally powered.

Under this working hypothesis, we can gather together the information from Table 4 to obtain a total global SFR of $7.5 M_{\odot} \mathrm{yr}^{-1}$ for SQ in its entirety. This global SFR does not seem particularly discrepant from that typically found for most of the galaxies of similar mass in the local universe that are clearly not so strongly interacting as SQ galaxies. Using the empirical relation between galaxy stellar mass and SFR for field galaxies shown in Figure 17 of Brinchmann et al. (2004), we can estimate the typical SFR of galaxies having the same stellar mass of SQ galaxies, of order of $\approx 10^{11} M_{\odot}$ (see Table 5$)$. For this value of the stellar mass, the mode of the distribution is at $\mathrm{SFR} \approx 1 M_{\odot} \mathrm{yr}^{-1}$. Therefore, since we estimated the SFR in a field containing four galaxies, the expected SFR would be $\approx 4 M_{\odot} \mathrm{yr}^{-1}$, comparable to the measured value. Thus, it seems that the star formation efficiency of SQ in relation to field galaxies is largely independent of whether the gas is inside or outside the main stellar disk. In fact, of the total global SFR of $7.5 M_{\odot} \mathrm{yr}^{-1}$ for SQ, 2.2 and $5.3 M_{\odot} \mathrm{yr}^{-1}$ can, respectively, be ascribed to the SED components for star formation regions and X-ray sources in Figure 19. This is a very remarkable result indicating, as it does, that the bulk of star formation activity in SQ is apparently associated with X-ray-emitting structures, occurring far away from the galaxy centers, either at the peripheries of the galaxies or in the IGM. Furthermore, the total extragalactic SFR is well in excess of the SFRs of the previously studied individual examples of extragalactic compact star formation regions, SQ A and SQ B.

To quantify the efficiency of star formation in the various components of SQ in relation to that of the disks of individual galaxies, we plotted in Figure 20 the SFR per unit area for dust emission sources in SQ as a function of the gas mass surface density. On the same diagram, as reference, we plotted the relations found by (Kennicutt et al. 2007, hereafter K07) and (Bigiel et al. 2008, hereafter B08) for star formation regions inside nearby spiral galaxies. This figure shows a wide range of star formation efficiencies, which we discuss below for each of the structural components, with special emphasis on the extended component of star formation which dominates the global SF activity in SQ. 


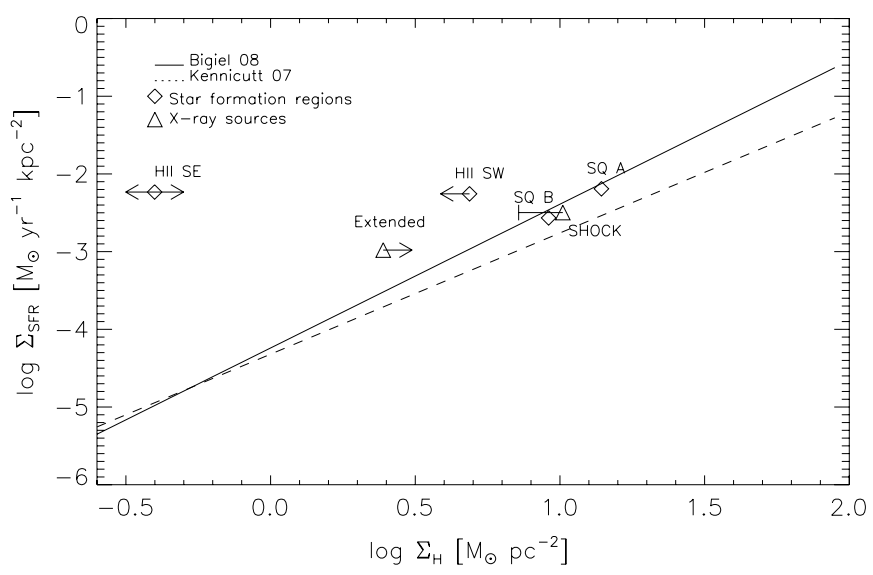

Figure 20. Star formation rate per unit area $\Sigma_{\mathrm{SFR}}\left(M_{\odot} \mathrm{yr}^{-1} \mathrm{kpc}^{-2}\right)$ vs. total gas mass surface density $\Sigma_{\mathrm{H}}\left(M_{\odot} \mathrm{pc}^{-2}\right)$ for dust emission sources in SQ. The plotted lines are the relations found by Kennicutt et al. (2007) and Bigiel et al. (2008). The arrows pointing toward right/left for some of the plotted measurements indicate that the corresponding $\Sigma_{\mathrm{H}}$ value is a lower/upper limit (see Table 4). In the case of $\mathrm{H}$ II-SE, the plotted gas surface density corresponds to the upper limit of the atomic hydrogen column density but the total gas density can also be higher since the amount of molecular gas is unknown. In the case of the shock region point, whose $\Sigma_{\mathrm{H}}$ value is the sum of both cold and hot gas components, a bar extends toward the left until the point where $\Sigma_{\mathrm{H}}$ corresponds to the cold gas mass surface density for this source.

\subsubsection{Discrete Star Formation Regions}

Figure 20 shows that SQ A and SQ B have SFRs very similar to those observed for galaxian regions with the same gas column density, despite their being located well outside the galaxies of the group. This result is consistent with the conclusions reached by Braine et al. (2001), studying a sample of tidal dwarf galaxies, and by Boquien et al. (2009), which performed a multiwavelength analysis of star formation regions in collisional debris. The situation is however different for the other two bright star formation regions, $\mathrm{H}$ II-SE and $\mathrm{H}$ II$\mathrm{SW}$, which present much higher SFRs than those predicted by the plotted relations. This might in principle be due to a more efficient mode of star formation happening in those regions. It would be of interest to acquire more complete information on the gas content of these sources to further investigate this conjecture. An alternative explanation for the high SFR found in $\mathrm{H}$ II-SE and $\mathrm{H}$ II-SW is that there is an additional component of UV emission produced by the radiative cooling of shocked gas. These UV photons, unrelated to star formation phenomena, can in principle contaminate the UV flux measurement but also power part of the observed dust emission, thus leading to an overestimation of the SFR. Optical spectra of these regions show evidences of line shock excitation (P.-A. Duc \& Collaborators 2010, private communication), therefore one cannot rule out this possibility.

\subsubsection{Star Formation Associated with the Shock}

The average SFR in the shock region $\left(3.1 \times 10^{-3}\right.$ $M_{\odot} \mathrm{yr}^{-1} \mathrm{kpc}^{-2}$ ) seems to be well in agreement with the empirical SFR-gas surface density relation in Figure 20. Thus, at first sight, the shock does not seem to have had much effect on the star formation activity in the stripped interstellar gas, neither triggering enhanced star formation through shock compression of dense clouds of gas, nor suppressing star formation through heating and dispersion of the clouds. The observational situation is however complex in that the star formation observed toward this region could be happening inside the extended features connected with the incoming galaxy NGC 7318b, seen through, but not obviously physically coexistent with the shock region. This is supported by the local morphology of the UV and MIR emission that seems to follow the optical shape of the intruder, instead of showing a linear north-south ridge resembling the shocked gas emission morphology. These two scenarios could in principle be combined since the ISM of the intruder galaxy has presumably been shocked as well and this could have triggered the observed star formation out of the gas associated with the intruder galaxy. In addition, it is conceivable that some fraction of the UV luminosity, albeit probably a minority, could be due to gas cooling rather than from massive stars.

It is also of interest to compare our constraints on the SFR in the shock region with the luminosity of the radio synchrotron emission from the shocked gas. Traditionally radio synchrotron measurements are compared to infrared emission measurements in the context of the radio-FIR correlation for individual galaxies, for which we use here the relation given by Pierini et al. (2003):

$$
\log L_{1.4 \mathrm{GHz}}=1.10 \times \log L_{\mathrm{FIR}}-18.53,
$$

where $L_{1.4 \mathrm{GHz}}$ is the luminosity at $v=1.4 \mathrm{GHz}$ in units of $\mathrm{W} \mathrm{Hz}^{-1}$ and $L_{\mathrm{FIR}}$ is the total FIR luminosity in units of W. We used the relation from Pierini et al. (2003) since it was derived from data covering cold dust emission longward of $>100 \mu \mathrm{m}$, where, as in the case for the SQ shock region, most of the power is radiated. Using the value we measured for the shock region FIR luminosity, $L_{\mathrm{FIR}}=1.5 \times 10^{36} \mathrm{~W}$, we obtain for the predicted radio luminosity $L_{1.4 \mathrm{GHz}}=1.8 \times$ $10^{21} \mathrm{~W} \mathrm{~Hz}$. This value is 20 times smaller than the radio luminosity derived from the radio measurement in $\mathrm{Xu}$ et al. 03: $L_{1.4 \mathrm{GHz}}=3.7 \times 10^{22} \mathrm{~W}$ Hz. The high radio/infrared luminosity ratio (which would be higher still with respect to the photonpowered component if collisional heating was important) points to the existence of an additional source of relativistic particles in the SQ shock, accelerated at the shock itself (see, e.g., Blandford \& Eichler 1987), which dominates the population of particles accelerated in sources more directly linked to star formation regions such as supernova remnants. This confirms and strengthens the preliminary results of Xu et al. (2003), and, bearing in mind that the total radio emission from SQ is dominated by the emission from the shock region, suggests that caution is needed in using radio synchrotron measurements to infer SFRs in groups involving strong dynamical interactions of galaxies with the IGM.

\subsubsection{Star Formation Associated with the Extended Emission}

Our measurements have shown that both the obscured and visible components of the star formation in SQ are distributed in a widespread pattern, loosely coincident both with the overall dimensions of the group as well as with the extended "halo" of X-ray emission. This current morphology of SQ star formation can readily be accounted for in terms of the several galaxy-galaxy or galaxy-IGM interactions which have occurred in the group. In this standard scenario, the gas that is currently converted into stars is interstellar in origin (as we will discuss later in this section). However, the data are also consistent with a rather different scenario, in which the reservoir of gas out of which stars are being formed is the hot gas phase of the intragroup medium.

Specifically, we consider a scenario in which the hot IGM is cooling and condensing into clouds which are the sites of the star formation providing the UV powered component of the 
extended infrared emission. If this is occurring in a steady state, in which the rate at which the removal of hot gas by cooling is balanced by accretion of further primordial gas (that is, we assume that variation in the accretion rate have longer timescales than the cooling timescale), we can write

$$
\mathrm{SFR}_{\text {hot }} \leqslant \frac{M_{\text {hot }}}{\tau_{\text {hot }}},
$$

where $M_{\text {hot }}$ and $\tau_{\text {hot }}$, respectively, denote the mass and cooling timescale of the X-ray-emitting medium, and the inequality denotes the fraction of the cooling gas that ultimately condenses into stars. If the observed X-ray emission is the main component of luminosity of the hot medium, and taking observational values for the X-ray-emitting plasma from Trinchieri et al. (2005) of $M_{\text {hot }}=1.0 \times 10^{10} M_{\odot}$ and $\tau_{\text {hot }} \approx 0.3 \times 10^{10} \mathrm{yr}$ (see Section 7.2.2), we obtain $\mathrm{SFR}_{\text {hot }} \leqslant 3 M_{\odot} \mathrm{yr}^{-1}$, comparable to the observed value of $5.3 M_{\odot} \mathrm{yr}^{-1}$ for star formation seen toward the X-ray-emitting halo. In reality, however, one would expect a large fraction of the cold gas resulting from the cooling of the gas to be recycled into the hot medium due to the feedback of mechanical energy from the newly formed stars, in which case $\mathrm{SFR}_{\text {hot }}$ would be substantially less the upper limit from Equation (8). Thus, if X-ray emission driven by gas-gas collisions is the dominant cooling path for the $\mathrm{X}$-ray halo, one would conclude that the bulk of the star formation would not be directly fueled out of the IGM. On the other hand, as shown in Section 7.2.2, our Spitzer FIR data, coupled with astrophysical constraints on the injection rate of grains into the hot medium do not at present rule out the possibility that the dominant cooling mechanism of the halo is FIR emission driven by gas-grain collisions. At present, therefore, we cannot rule out star formation out of a primordial IGM purely on considerations of gas fueling.

A more powerful constraint would be to consider the efficiency at which such a mode would have to operate at. Unfortunately, the total mass of molecular cold gas, $M_{\text {cold }}$ on a global scale in the halo is unknown, preventing a direct empirical measurement of star formation efficiency of the halo on the Kennicutt-Schmidt (KS) diagram. One can, however, estimate $M_{\text {cold }}$ under our simple steady state scenario by writing

$$
\frac{M_{\text {hot }}}{\tau_{\text {hot }}} \approx \frac{M_{\text {cold }}}{\tau_{\text {cold }}},
$$

where $\tau_{\text {cold }}$ is the typical timescale spent by the gas in the cold molecular phase before being converted into stars, which is the timescale for the collapse of a molecular clouds to form stars, multiplied by the mean number of times the cold gas is recycled into the hot medium through mechanical feedback before condensing into a star. Even allowing for several cooling cycles of the hot gas before condensing into stars, the very short collapse timescale of a few million years for molecular clouds-some 3 orders of magnitude shorter than $\tau_{\text {hot }}$-makes it likely that $\tau_{\text {hot }} \gg \tau_{\text {cold }}$, which in turn implies $M_{\text {hot }} \gg M_{\text {cold }}$. If this is the case, the cold gas amount, related to the extended emission, that we should add to $\Sigma_{\text {gas }}$ would be negligible, and the position of the point for the SQ halo on the KS diagram would be well to the left of the relation for star-forming galaxies, as shown in Figure 20. This would require a more efficient star formation process in the IGM than is typically observed to occur in the disks of star-forming field galaxies, which would seem to be counterintuitive to the naive expectation that denser, colder reservoirs of gas closer to the minimum of the gravitational potential wells associated with individual galaxies should form stars more easily. On the other hand, a definition of efficiency in terms of gas surface density over scales much larger than the star formation regions may have limited predictive power in this context. Furthermore, any star formation in a cooling hot IGM would not be expected to occurring in rotationally supported systems similar to the galaxies used to define the KS relation, so may have a different relation to the local gas density.

The second, more conventional scenario to explain the widespread star formation in the extended halo component of $\mathrm{SQ}$, is to invoke interstellar gas as the source of cold gas fueling most of the star formation. In this scenario, tidal interactions between galaxies or hydrodynamical interactions of the ISM with the IGM remove cold galaxy material that, for $S Q$, produces a similar SFR as would have been the case for a similar amount of gas inside field galaxies. For groups in general the statistical relation of neutral gas observed within and outside the member galaxies to the X-ray emission characteristics of the IGM indeed indicates that at least some of the cold gas in the IGM medium originates in the galaxies, and that at least some of the X-ray-emitting gas also has an interstellar origin (e.g., VerdesMontenegro et al. 2001; Rasmussen et al. 2008). More direct evidence of the removal of interstellar gas and dust through galaxy interactions in high density environments is provided by observations of interacting galaxies in clusters, for example, through Herschel imaging of extraplanar dust emission associated with stripped atomic and molecular gas around the Virgo cluster galaxy NGC 4438 (Cortese et al. 2010).

In SQ, there is direct morphological evidence for bursting sources associated with tidally removed interstellar gas in the form of SQ A and SQ B, and a hint that other less prominent components of the FIR emission in the IGM may also be associated with tidal features, such as the enhancement of $\mathrm{H}_{\alpha}$ and MIR/FIR emission seen toward the bridge linking the intruder galaxy with the AGN host galaxy. In this picture, the extended emission is a conglomeration of discrete sources similar in nature to SQ A and SQ B but much more numerous and of lower power. The fact that SQ A and SQ B are likely to have a fundamentally interstellar origin despite their present location outside the galaxies is further supported by the rather high value of the dust-to-gas ratio we have found for these sources, $Z_{\mathrm{d}}=0.002$, which is a signature of a high gas metallicity. The one puzzling aspect of the extension of this scenario to the global star formation in the IGM of SQ is the lack of prominent star formation associated with the bulk of the H $\mathrm{I}$ in the IGM located to the south and east of the group. However, as shown by Figure 5 of Williams et al. (2002), the gas column density in these clouds (generally less than $\approx 3 \times 10^{20}$ atoms $\mathrm{cm}^{-3}$ ) is rather low compared to the values found for the brightest star formation sites in SQ. Therefore, it is plausible that star formation is inhibited in these clouds because of the low gas density.

Finally, we remark that if stripped neutral and molecular interstellar gas was the only source of cold gas powering star formation, one would expect a rapid quenching of star formation activity in SQ in the future, whereas if the star formation were fueled by a cooling primordial IGM, the star formation activity would simply follow the accretion rate onto the group of the primordial IGM. Thus, measurement of the UV and MIR/FIR luminosity functions of well-defined statistical samples of galaxy groups, now being defined through deep optical spectroscopical surveys such as the Galaxy And Mass Assembly survey (GAMA; Driver et al. 2009), may offer a way of quantifying the relative importance of these modes of star 
formation on the dynamical halo mass of groups in the local universe.

\subsection{The Nature of the X-ray Correlated FIR Emission in Stephan's Quintet}

Previous searches for infrared emission counterparts of the hot X-ray-emitting components of the IGM of nearby objects have almost exclusively targeted the ICM of rich galaxy clusters, either using a stacking analysis for X-ray or optical selected clusters from the IRAS all sky survey (Giard et al. 2008; Roncarelli et al. 2010) or in detailed imaging observations of the Coma Cluster and other clusters with ISO or Spitzer (Stickel et al. 1998, 2002; Quillen et al. 1999; Bai et al. 2007; Kitayama et al. 2009). All these studies have either yielded upper limits or marginal apparent detections of the ICM at far-IR brightness levels far fainter than those we have measured toward the X-ray-emitting IGM of SQ with Spitzer. Furthermore, even the apparent detections of the ICM were susceptible to confusion with foreground cirrus or the background galaxy population due to the accidental similarity (Popescu et al. 2000) in far-IR color of the collisionally and photon-heated emissions, so realistically must be treated as upper limits to any collisionally heated emission from the ICM (Bai et al. 2007). Although intracluster dust has been unambiguously detected at optical wavelengths, through statistical studies of the reddening of background sources through large numbers of clusters (Chelouche et al. 2007 ) this has been at a low abundance of about $10^{-4}$ in the dust-to-gas mass ratio, consistent both with the non-detections of the ICM in infrared emission and with specific predictions by Popescu et al. (2000) of the dust content of typical ICMs.

In the present work, we have been able to sidestep the confusion problems afflicting the previous attempts to detect infrared emission from the ICM in clusters only by virtue of the much higher far-IR brightness levels measured toward the X-ray-emitting structures in SQ. These high brightness levels, together with the correspondence of the infrared emission morphologies with those seen in the UV, and the evidence from our fits to the infrared emission SEDs described in Section 5.3 however suggest that a major part of the dust emission is photon heated. This, in turn, raises an apparent paradox that the closest spatial correlation between dust emission and gas column density is not, as might be expected for photon heating, with the cool $\mathrm{HI}$ and molecular gas component, but rather with the hot X-ray-emitting component. In our discussion on star formation in SQ, we identified a possible way out of this paradox by postulating that the extragalactic star formation in SQ is fueled by gas from a hot IGM, whose cooling is enhanced by grains injected into the hot IGM. Here, we use the relative levels of detected emission at infrared, X-ray, and UV/optical wavelengths to constrain the possible sources of grains and the resulting collisionally driven cooling of the hot plasmas, considering separately the two main X-ray-emitting structures corresponding to the shock and the halo regions.

\subsubsection{The Shock Region}

The main constraint on the collisional heating of dust in the $\mathrm{X}$-ray-emitting plasma downstream of the shock is given by the high dust-to-gas mass ratio that would be required to reproduce the FIR measurements in the case that grain heating by a diffuse radiation field can be neglected. For pure collisionally heated emission from grains in a homogeneous medium, a dust mass $M_{\mathrm{d}}=2 \times 10^{7} M_{\odot}$ (see Section 5.3) would be required, which, taken together with the measured X-ray-emitting gas mass, $M_{\mathrm{X}} \approx 10^{9} M_{\odot}$ (see Appendix $\mathrm{C}$ ), would imply a dustto-gas ratio for the hot plasma of $Z_{\mathrm{d}}=0.02$. This value is high in comparison with expectations based on a balance between injection of grains and their removal through sputtering. Assuming that the shock is propagating into a stripped ISM or through the ISM of the intruder galaxy, characterized by a dust-to-gas ratio comparable to the Milky Way value, we can derive the amount of dust mass per unit time $\dot{M}_{\mathrm{D}}$ that is injected downstream of the shock:

$$
\dot{M}_{\mathrm{D}}=V_{\mathrm{sh}} A \rho_{\mathrm{up}} Z_{\mathrm{d}, \mathrm{up}}
$$

where $V_{\mathrm{sh}}=600 \mathrm{~km} \mathrm{~s}^{-1}$ is the shock velocity (Guillard et al. 2009), $A=330 \mathrm{kpc}^{2}$ is the projected area of the shock front (corresponding to $20 \times 80 \operatorname{arcs}^{2}$ on the sky), $\rho_{\text {up }} \approx 1.1 n_{\mathrm{H}} m_{\mathrm{H}}=4.5 \times 10^{-27} \mathrm{~g} \mathrm{~cm}^{-3}$ is the upstream gas density $\left(n_{\mathrm{H}}=0.01 / 4 \mathrm{~cm}^{-3}\right)$ and $Z_{\mathrm{d} \text {,up }}=0.01$ is the assumed dust-to-gas ratio for the gas upstream of the shock front. The resulting dust injection rate is $\dot{M}_{\mathrm{D}}=0.14 M_{\odot} \mathrm{yr}^{-1}$. To estimate grain destruction through sputtering in collisions with heavy particles, we use the formula of Draine \& Salpeter (1979) for the dust sputtering timescale:

$$
\tau_{\mathrm{sp}}=2 \times 10^{6} \frac{a}{n_{\mathrm{H}}} \mathrm{yr},
$$

where $a$ is the dust size in $\mu \mathrm{m}$ and $n_{\mathrm{H}}$ is the proton number density in $\mathrm{cm}^{-3}$. Assuming $n_{\mathrm{H}} \approx 0.01 \mathrm{~cm}^{-3}$ and $a=0.1 \mu \mathrm{m}$, we get $\tau_{\mathrm{sp}}=2 \times 10^{7} \mathrm{yr}$. Explicit calculation of the cooling of the shock gas due to gas-gas and gas-grain collisions, taking into account the evolution of the grain size distribution (under the assumption that there are no further sources of grains), shows that the gas cooling timescale is $t_{\text {cool }} \approx 7 \times 10^{7} \mathrm{yr}$, more than three times larger than $\tau_{\mathrm{sp}}$ (Natale 2010). Under these circumstances, a conservative estimate for the dust-to-gas ratio downstream of the shock can be derived by dividing the total mass of dust predicted to be at any time downstream of the shock to the measured hot gas mass, that is,

$$
Z_{\mathrm{d} \text { down }}=\frac{\dot{M}_{\mathrm{D}} \tau_{\mathrm{sp}}}{M_{\mathrm{X}}} .
$$

Applying this formula, the expected value for the X-ray plasma dust-to-gas ratio is $Z_{\mathrm{d} \text {, down }} \approx 3 \times 10^{-3}$, a factor of $\approx 7$ smaller than the value inferred from the data, for the case where the entire FIR dust emission is powered by collisional heating. This is only a crude estimate for the dust abundance, which might take higher values if the line-of-sight depth of the shock was assumed to be larger than the projected width seen on the sky, but which on the other hand is an overestimate compared to predictions taking into account the true evolution of grain size downstream of the shock. In any case, it is evident that the measured dust-to-gas ratio is much larger than the predicted $Z_{\mathrm{d}, \text { down }}$, and a further source of dust grains would be required if collisional heating were to be a significant driver of the FIR emission.

The most obvious such source would be the reservoir of grains in the cold component of the gas in the shock region, which could be potentially released into the hot gas by ablation if the clouds were in relative motion to the hot gas. As already mentioned, the presence of cold gas is implied by the detection of $8 \mu \mathrm{m}$ emission and has been directly demonstrated by the detection of molecular hydrogen in the MIR rotational lines (Cluver et al. 2010). Guillard et al. (2009) modeled the $\mathrm{H}_{2}$ emitting clouds as 
cooling pre-existing clouds in the upstream medium, a scenario which would predict the clouds to be in relative motion to the hot gas downstream of the shock. Another possibility which might potentially account for an FIR counterpart to the shock is to invoke heating of grains upstream of the shock by the UV radiation from the cooling clouds downstream of the shock. A quantitative treatment of all these effects would however require a self-consistent model for the passage of the shock through a twophase medium which tracked the exchange of photons, gas, and dust between the phases, which is beyond the scope of this paper.

It is appropriate to emphasize here that even in the case that the collisional heating is relatively unimportant in bolometric terms, the effect on the gas cooling timescale is not negligible. Detailed calculations for a steady state one-dimensional homogeneous model, considering dust sputtering and dust cooling, show that the cooling timescale is shorter by a factor of $\approx 2-3$ (Natale 2010) for shock velocities and gas densities similar to those characteristic of the shock in SQ. From this perspective, it would be important to have more direct empirical constraints on the gas cooling mechanism using the improved sensitivity, angular resolution, and wavelength coverage of the Herschel Space Observatory. Using the simple approach described above, we estimate the surface brightness of the radiation emitted by collisionally heated dust to be equal to $1.4 \mathrm{MJy} \mathrm{sr}^{-1}$ at $150 \mu \mathrm{m}$, the wavelength where the flux density is predicted to peak according to the collisional-heated dust model we used for the shock region SED fit (see Figure 16).

\subsubsection{The Extended Emission}

As for the shock, the detection of substantial $8 \mu \mathrm{m}$ emission toward the extended X-ray-emitting halo indicates the presence of a component of dust in cold gas phases, shielded from collisions with electrons and ions in the hot plasma, which can only be heated by photons. Rather interestingly, the curves of growth we constructed after removing or masking the galaxies and the compact star formation regions from the infrared and FUV maps (Figure 10) show a very similar profile at all wavelengths. This is a strong indication that FUV sources are associated with the FIR extended emission and, therefore, star formation is the main mechanism powering the extended emission.

The total luminosity of the extended dust emission is $4 \times$ $10^{43} \mathrm{erg} \mathrm{s}^{-1}$, a factor $\approx 130$ higher than the X-ray halo luminosity (Trinchieri et al. 2005; see also Appendix C). This large difference between the dust emission and X-ray luminosities implies that collisional heating of dust grains could still be important for the cooling of the hot halo gas even if it only powers a small fraction of the dust luminosity. We define the fraction $F_{\text {coll }}$ of dust emission that is collisionally powered, by the relation

$$
L_{\mathrm{IR}}^{\text {coll }}=F_{\text {coll }} L_{\mathrm{IR}},
$$

where $L_{\mathrm{IR}}^{\text {coll }}$ is the dust luminosity that is powered by collisional heating.

A first physical constraint on $F_{\text {coll }}$ that we can apply is the requirement that the gas cooling timescale $\tau_{\text {cool }}$ is not larger than the dynamical hot gas timescale, given by the sound crossing time $\tau_{\text {cross. }}$. Including dust cooling, $\tau_{\text {cool }}$ is equal to

$$
\tau_{\text {cool }}=\frac{3}{2} \frac{k T}{\mu m_{\mathrm{H}}}\left(\frac{M_{\mathrm{X}}}{L_{\mathrm{X}}+F_{\mathrm{coll}} L_{\mathrm{IR}}}\right),
$$

where $T=6 \times 10^{6} \mathrm{~K}$ is the gas temperature, $M_{\mathrm{X}}=10^{10} M_{\odot}$ is the X-ray-emitting gas mass, and $L_{\mathrm{X}}$ and $L_{\mathrm{IR}}$ are the $\mathrm{X}$-ray and infrared luminosities. Requiring that $\tau_{\text {cool }}>\tau_{\text {cross }}=2 R_{\text {halo }} / c_{s}$, where $R=35 \mathrm{kpc}$ is the halo radius and $c_{s} \approx 300 \mathrm{~km} \mathrm{~s}^{-1}$ is the sound speed, one obtains $F_{\text {coll }}<0.05$.

Another constraint can be derived from an estimation of the dust injection rate into the hot gas. For equilibrium between dust injection and sputtering, one would have

$$
\dot{M}_{\mathrm{d}}=F_{\text {coll }} \frac{M_{\mathrm{d}}}{\tau_{\mathrm{sp}}}=2.4 F_{\text {coll }} \frac{\mathrm{M}_{\odot}}{\mathrm{yr}},
$$

where $\dot{M}_{\mathrm{d}}$ is the dust injection rate, $\tau_{\mathrm{sp}}=2 \times 10^{8} \mathrm{yr}$ is the sputtering timescale (calculated using formula (11) with $n_{\mathrm{H}}=0.001 \mathrm{~cm}^{-3}$ and $\left.a=0.1 \mu \mathrm{m}\right)$, and $M_{\mathrm{d}}=4.7 \times 10^{8} M_{\odot}$ is the inferred dust mass producing the extended emission.

The first dust injection sources we consider are the halo stars. As described in Appendix E, we have derived an upper limit to the dust injection rate from halo stars based on the observed $R$-band halo surface brightness (Moles et al. 1998) and the theoretical predictions for stardust injection by Zhukovska et al. (2008): $\dot{M}_{\mathrm{d}}=0.075 M_{\odot} \mathrm{yr}^{-1}$. Substituting this value in Equation (15), we get $F_{\text {coll }}<0.03$. A similar procedure could be followed considering the dust injection from type II supernovae given the inferred SFR associated with the extended emission. Empirical studies of Galactic supernova remnants have shown the yield of condensate per supernova event to be of the order of 0.01-0.1 $M_{\odot}$ (Green et al. 2004; Fischera et al. 2002). Relating the frequency of core collapse supernova events to the SFR in the extended component of SQ, we estimated $\dot{M}_{\mathrm{d}}=7 \times 10^{-4} M_{\odot} \mathrm{yr}^{-1}$ assuming $0.01 M_{\odot}$ of dust produced in each supernova. Therefore, supernovae dust injection cannot be considered important in replenishing dust in the hot medium. Finally, we considered the mechanism proposed by Popescu et al. (2000) where dust in IGM external to a cluster can be introduced into the hot ICM in the supersonic accretion flow of barions onto the cluster. In the case of SQ, the baryonic accretion rate can be estimated to be of the order of the current IGM seen in X-ray divided by a Hubble time which is $\approx 2 M_{\odot} \mathrm{yr}^{-1}$. Even if we consider that there is maybe a cooler component of the accreting IGM not visible in the X-ray (Mulchaey 2000), it does not seem plausible to achieve dust accretion rate for the particular case of SQ much greater than $0.01 M_{\odot} \mathrm{yr}^{-1}$ for reasonable values of the dust-to-gas ratio in the accreting material.

Given these estimates, we can conclude that if there is a contribution of collisional-heated dust to the total observed dust emission, this should only be of the order of a few percent and maintained by direct injection from the in situ stellar population. This however is sufficient to maintain a collisionally powered FIR luminosity of up to $\approx 10^{42} \mathrm{erg} \mathrm{s}^{-1}$, which is several times the X-ray luminosity.

Such a level of FIR cooling could help to provide a natural explanation for the rather low ratio of $\sim 0.02$ for the ratio of the mass of baryons in the hot X-ray-emitting halo of $\sim M_{\mathrm{X}}=2 \times 10^{10} M_{\odot}$ to the dynamical mass of $\sim 10^{12} M_{\odot} \cdot{ }^{18}$ For the concordance cold dark matter cosmology, this ratio

\footnotetext{
18 This value is derived from the X-ray temperature that is consistent with the velocity dispersion of the group, $\sigma=340 \mathrm{~km} \mathrm{~s}^{-1}$, as calculated by O'Sullivan et al. (2009). However, including the more recent recession velocity measurement for NGC 7320c, quoted in Section 4.1 of Sulentic et al. (2001), the group velocity dispersion would be $\sigma=80 \mathrm{~km} \mathrm{~s}^{-1}$ indicating a DMH mass of order of $10^{11} M_{\odot}$. Since the number of group galaxies is very low for a good estimate of the velocity dispersion, we consider the X-ray temperature based estimate for the $\mathrm{DMH}$ mass more reliable in this case.
} 
should be $\sim 0.09$ in the absence of significant dissipative effects (see, e.g., Figure 9; Frenk et al. 1999). Recalling that the total baryonic mass currently locked in stars in SQ of $\sim 10^{11} M_{\odot}$ is comparable to the expected total mass of baryons, it is apparent that this scenario is also quantitatively consistent with the efficient condensation of the IGM accreting onto the group into stars, as postulated in Section 7.1. Some empirical indication of efficient star formation in groups has been found by Tran et al. (2009), who showed that the group environment is more conducive to star formation than either the cluster or field environment. In general, enhanced cooling due to an FIR collisionally powered luminosity of a few times $L_{X}$ would, since it promotes the condensation of hot X-ray-emitting gas into stars, also provide an alternative explanation for the steeper $L_{\mathrm{X}}-T_{\mathrm{X}}$ relation observed for groups with lower virial velocities, an effect that is otherwise attributed to thermal or kinetic feedback from AGNs (e.g., Cavaliere \& Lapi 2008).

In summary, the detection and recognition of the putative collisionally heated component of FIR emission from SQ will be a crucial measurement with wide ranging implications for the nature of star formation in the group, the division of baryons between hot gas, cold gas and stars, and the thermodynamic properties of the IGM in SQ. Although such a detection will be very challenging, requiring a combination of excellent surface brightness sensitivity, spectral grasp, and angular resolution at submillimeter wavelengths, it could be achieved by the planned Space Infra-Red Telescope for Cosmology and Astrophysics (SPICA, Nakagawa 2010; Swinyard et al. 2009), as illustrated by the following estimates: assuming the upper limit $F_{\text {coll }}=0.03$, we predict the SED of the collisionally powered component of the extended emission to peak at a level of $0.15 \mathrm{MJy} \mathrm{sr}^{-1}$ at $190 \mu \mathrm{m}$.

\subsection{The AGN Galaxy NGC 7319 Dust Emission}

The Seyfert 2 galaxy NGC 7319 is the most powerful source of IR emission in SQ and is the only galaxy where we found enhanced infrared emission in the central regions. The total IR luminosity is $9.6 \times 10^{43} \mathrm{erg} \mathrm{s}^{-1}$. Of this, we deduced from the SED fitting using a combination of the AGN torus template and the diffuse dust emission component (see Section 5.2) that $4.1 \times 10^{43} \mathrm{erg} \mathrm{s}^{-1}$ are emitted by dust in the torus and $5.5 \times 10^{43} \mathrm{erg} \mathrm{s}^{-1}$ by diffuse dust. As one can see from the SED best fit, shown in Figure 15, the $24 \mu \mathrm{m}$ MIR emission is dominated by the torus emission which also contributes $\approx 25 \%$ of the $8 \mu \mathrm{m}$ emission. This is consistent with the observed morphology at 8 and $24 \mu \mathrm{m}$ emission, which is a combination of a central source plus a disk component in the case of the $8 \mu \mathrm{m}$ map and a dominant central source in the case of the $24 \mu \mathrm{m}$ map (see Figure 1). According to the SED fit, the FIR 70 and $160 \mu \mathrm{m}$ emission is due to diffuse dust emission. This is also consistent with the brightness distributions on the Spitzer FIR maps which show the emission to be partially resolved, with extents inferred from the FIR map fitting technique of 5 and $12 \mathrm{kpc}$ at 70 and $160 \mu \mathrm{m}$, respectively. This extended FIR emission is centered at the position of the AGN, potentially indicating the presence of star formation regions distributed on kpc scales in the surrounding areas. However, as one can see from Figure 1 of Gao \& Xu (2000), the CO emission is predominantly from the northern regions of this galaxy. Therefore, the locations of the bulk of cold gas, where the star formation sites should be, and the cold dust emission are not coincident suggesting that star formation may not be the main phenomena powering the FIR dust emission.
Overall, the diffuse dust emission in the inner disk, together with the absence of $\mathrm{HI}_{\mathrm{I}}$ (Williams et al. 2002) and the displacement of the molecular hydrogen emission suggest that galaxy-galaxy interactions have removed gas from the central regions on kpc scales, as has plainly also happened in the other late-type SQ galaxies. In the case of NGC 7319, the interactions have apparently also induced gas flows that have triggered AGN activity. This raises the question whether the AGN itself might be the source of photons powering the surrounding diffuse dust emission in this galaxy. According to the standard AGN unification theory, the AGN torus of a Seyfert 2 galaxy is viewed edge-on, which, in the case of NGC 7319, would imply that the plane of the torus is almost perpendicular to the galaxy disk, since NGC 7319 is seen basically face-on (see SDSS $g$-band map in Figure 1). In this configuration, it would indeed be plausible that accretion powered radiation, escaping into the polar direction from the AGN torus structure could propagate into the galaxy disk and contribute to the diffuse radiation field in the central parts of the galaxy. An especially efficient coupling between the dust and radiation would be expected if the polar axis of the AGN torus were to be parallel to the diffuse dusty disk of the host galaxy.

One indirect indication that the radiation from an AGN with this orientation may be responsible for at least part of the diffuse FIR emission in NGC 7319 comes from the rather low observed $\mathrm{UV} /$ FIR luminosity ratio, which is qualitatively consistent with the expected strong attenuation of the direct UV light from an AGN to which the line of sight passes through the torus. From the measured FUV luminosity, $L_{\mathrm{FUV}}=4 \times 10^{42} \mathrm{erg} \mathrm{s}^{-1}$, and from the 60 and $100 \mu \mathrm{m}$ luminosities extrapolated from the SED best fit, $L_{60 \mu \mathrm{m}}=2 \times 10^{43} \mathrm{erg} \mathrm{s}^{-1}$ and $L_{100 \mu \mathrm{m}}=3.7 \times 10^{43} \mathrm{erg} \mathrm{s}^{-1}$, we have found $L_{\mathrm{FUV}} / L_{60 \mu \mathrm{m}}=0.2$ and $L_{\mathrm{FUV}} / L_{100 \mu \mathrm{m}}=0.1$. These values are several factors lower than the average values found by Popescu \& Tuffs (2002) for galaxies of similar morphological type $\left(L_{\mathrm{UV}} / L_{60 \mu \mathrm{m}}=1\right.$ and $\left.L_{\mathrm{UV}} / L_{100 \mu \mathrm{m}}=0.6\right)$ and by Xu et al. (2006) for galaxies of similar $L_{\mathrm{FUV}}+L_{60 \mu \mathrm{m}}$ total luminosity $\left(L_{\mathrm{UV}} / L_{60 \mu \mathrm{m}}=0.5\right)$.

A scenario in which the diffuse dust emission in NGC 7319 is powered by the AGN also seems to be broadly consistent with simple constraints on the UV luminosity of the AGN derived from the observed hard X-ray luminosity. Specifically, for $L_{2-10 \mathrm{keV}}=2.8 \times 10^{42} \mathrm{erg} \mathrm{s}^{-1}$ (Trinchieri et al. 2005) and adopting the bolometric corrections for AGN galaxies with similar $L_{2-10 \mathrm{keV}}$ by Vasudevan et al. (2010), $\kappa_{2-10 \mathrm{keV}} \approx 10-30$, one predicts a total UV luminosity from the AGN of $L_{\mathrm{AGN}}=$ $\kappa_{2-10 \mathrm{keV}} L_{2-10 \mathrm{keV}}=(2.8-8.4) \times 10^{43} \mathrm{erg} \mathrm{s}^{-1}$. The upper end of this range is comparable to the total MIR/FIR luminosity of the AGN and AGN host galaxy. If the AGN were indeed powering the diffuse dust emission in the inner disk one would expect that future FIR observations of angular resolution sufficient to image the diffuse dust emission could be used to determine some basic geometrical properties of the AGN. Specifically, one would expect a cone structure for the component of diffuse dust heated by the AGN, with a strong radial color gradient, and with a symmetry axis aligned with the polar axis of the torus.

Finally, we draw attention to a further FIR-emitting feature potentially connected to the AGN, whose origin has still to be clarified. This is the bridge which apparently connects the AGN to the center of the shock region, appearing in the X-ray (Trinchieri et al. 2005), $\mathrm{H} \alpha$ (Xu et al. 1999), and warm $\mathrm{H}_{2}$ line emission (Cluver et al. 2010). The presence of a cold dust emission from this feature is hinted by a slight asymmetry of the 
FIR emission on the $160 \mu \mathrm{m}$ residual map (see Figure 4). Highresolution FIR observations would also be useful to elucidate the correspondence between dust emission and the gas distribution in this bridge feature.

\section{SUMMARY}

In this work, we have performed an extensive analysis of the infrared emission from SQ, based on the Spitzer MIR and FIR maps and including multiwavelength data from optical/ $\mathrm{UV}$, radio, and X-ray. The main aim has been understanding the nature of the FIR emission seen on the Spitzer 70 and $160 \mu \mathrm{m}$ maps and its implications for star formation and hot gas cooling in this group of galaxies. The technical part of our analysis can be summarized in four points.

1. We have identified the FIR sources seen on the Spitzer maps and performed their photometry on both the MIR and FIR maps. For the FIR photometry, we devised a new source fitting technique that we used to model the source emission on the maps (see Figures 2 and 3). We measured MIR fluxes using aperture photometry (see Figures 6 and 7). Photometry results are shown in Table 2.

2. We have compared the residual FIR emission (defined as the FIR emission after the subtraction of galaxies and discrete star formation regions) with $\mathrm{H}$ I distribution, X-ray emission, and UV emission (see Figure 4). This comparison shows that the residual FIR emission, dominated by the emission from the shock region and a previously undetected extended component, is correlated with X-ray emission and anticorrelated with the $\mathrm{HI}$ distribution. At the same time, the area covered by the FIR residual emission is populated by an extended distribution of UV sources associated with tidal features and intergalactic star formation regions.

3. We have modeled the inferred infrared source SEDs using combinations of dust emission templates. These templates, described in Appendix B, are used to reproduce emission from $\mathrm{PDR} / \mathrm{H}$ II regions, diffuse-photon-powered dust emission, collisionally powered dust emission, and infrared emission from AGN torus. Among the immediate outcomes of the fitting procedure, there are the dust mass required to produce the observed emission, the total dust infrared luminosity, and the amount of UV powered dust emission (see Table 3).

4. Combining the amount of absorbed UV luminosity, obtained from the infrared SED fitting, with the observed UV luminosity, measured directly on the GALEX FUV maps, we determined the SFR for each dust emitting source. We also measured gas masses/column densities from the observed radio $\mathrm{H}$ I and $\mathrm{CO}$ lines and soft X-ray fluxes and, combining these measurements with the dust masses inferred from the infrared SED fits, we estimated the dustto-gas ratios for several sources. Results are summarized in Table 4.

From this set of measurements and morphological comparisons, together with simple predictions for the gas cooling timescale and the dust injection rate in hot plasma, we derived the following conclusions.

1. The total SFR, within a circular area of radius $90^{\prime \prime}$ containing the four group galaxies in close proximity, is $7.5 M_{\odot} \mathrm{yr}^{-1}$. The star formation regions in the group are localized at the edges of the galaxies, on tidal features and in the IGM; no evidences for star formation have been found toward galaxy centers.
2. The star formation efficiency for the star formation regions SQ A and SQ B, localized on tidal features, is close to that observed for star formation regions within nearby spiral galaxy disks (see Figure 20). Star formation in H II-SE and H II-SW seems to be much more efficient but the lack of complete gas measurements and the possible contribution to UV radiation from radiative shocks does not allow a definite conclusion for these sources.

3. The star formation efficiency in the shock region seems to be consistent with the star formation observed in nearby spiral galaxies. However, because of the elongated tidal feature of the intruder galaxy NGC 7318b seen through the shock region, it is not clear if the observed star formation in that area is associated with the shocked gas or not. The observed radio-IR luminosity ratio is 20 times higher than the value predicted by the radio-infrared relation derived by Pierini et al. (2003), given the observed amount of infrared luminosity. This reinforces the result obtained by $\mathrm{Xu}$ et al. (2003), suggesting that the acceleration of relativistic particles in the shock region is not associated with star formation.

4. Seventy percent of the total star formation in SQ is associated with the extended FIR emission. The star formation efficiency for this component is rather higher than that observed in spiral galaxies. This could in principle be due to a new mode of star formation operating in the group and involving cooling of the X-ray halo gas, possibly enhanced by dust-plasma particle collisions. However, the lack of cold gas measurement for this distributed star formation component makes this hypothesis rather uncertain. In addition, the evidence of tidal interactions and star formation in tidally displaced cold gas, like SQ A or SQ B, suggests that the distributed star formation observed in the group is in part occurring out of gas of galaxian origin.

5. For both the shock region and the extended emission, we have measured substantial $8 \mu \mathrm{m}$ flux, mainly due to PAH molecule line emission and tracer of cold gas phases. This, together with simple estimates of the dust injection rate across the shock front and in the halo of the group, demonstrates that the mechanism powering most of the dust emission seen on the FIR residual maps is not dust collisional heating. However, given the high FIR-X-ray luminosity ratio, the current data do not exclude that the majority of the hot gas cooling can still be due to dust-plasma particle collisions rather than X-ray emission. For dust in the group halo, we estimated that dust collisional heating can power up to $10^{42} \mathrm{erg} \mathrm{s}^{-1}$ of the dust luminosity.

6. We have found that the morphology of the FIR dust emission in the Seyfert 2 galaxy NGC 7319 does not correspond to the cold gas distribution. Therefore, the FIR emission in this galaxy does not seem to be related to star formation. Given the AGN UV luminosity, estimated from the observed hard X-ray flux, and from considerations on the particular torus-galaxy disk geometry, we deduced that accretion powered radiation from the AGN, propagating through the disk of the galaxy, can power most of the observed dust infrared emission.

We thank Pierre Guillard for fruitful discussions on the shock region of SQ, and Francois Boulanger and Fabian Walter for discussions and comments on the manuscript. G.N. acknowledges support from the International Max-Planck Research School (IMPRS) Heidelberg. This work is based on observations made 

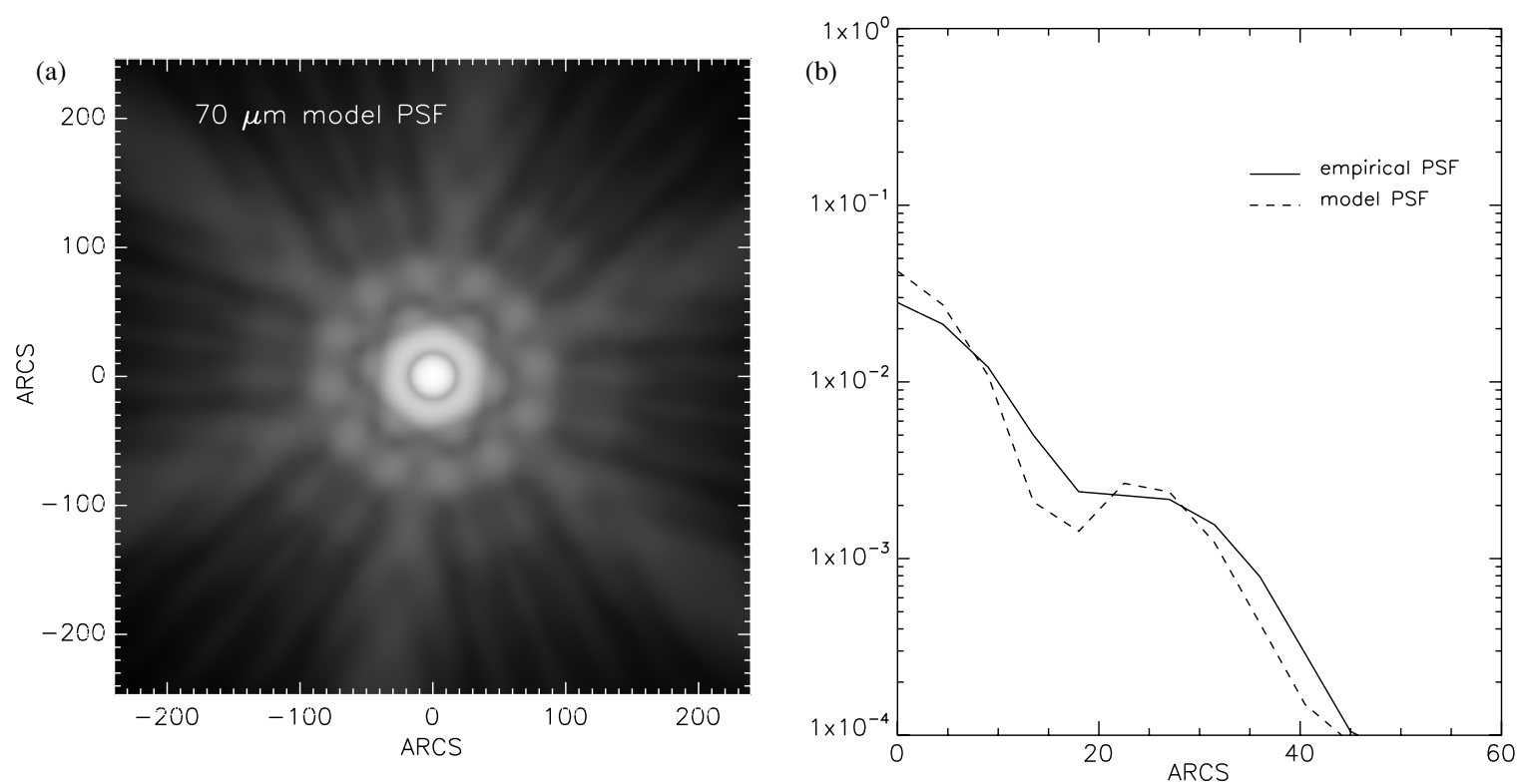

Figure 21. (a) Theoretical $70 \mu \mathrm{m}$ PSF and (b) average radial profiles empirical and theoretical $70 \mu \mathrm{m}$ PSF.

with the Spitzer Space Telescope, which is operated by the Jet Propulsion Laboratory, California Institute of Technology under a contract with NASA.

\section{APPENDIX A}

\section{MULTISOURCE FIT OF SQ FIR MAPS}

The technique we developed for the fitting of the Spitzer FIR maps is similar to the CLEAN algorithms used in radio astronomy but with some additional characteristics: (1) all the sources are fitted at the same time and (2) an intrinsic finite source width is allowed. Our basic assumption is that all the sources we model are sufficiently well described by elliptical Gaussians or a combination of them. This is not necessarily true because some sources can, in principle, differ significantly from this simple shape. However, this simple approach has been sufficient for a good fitting of the FIR maps. The function $F(x, y)$, describing an elliptical Gaussian on a map, is defined by the following formulae:

$$
\begin{gathered}
F(x, y)=B_{\mathrm{loc}}+A \times e^{-U / 2} \\
U=\left(x^{\prime} / \sigma_{x}\right)^{2}+\left(y^{\prime} / \sigma_{y}\right)^{2} \\
x^{\prime}=\left(x-x_{s}\right) \cos \theta-\left(y-y_{s}\right) \sin \theta \\
y^{\prime}=\left(x-x_{s}\right) \sin \theta+\left(y-y_{s}\right) \cos \theta .
\end{gathered}
$$

The first two equations define the shape of the elliptical Gaussian while the last two are the coordinate transformations between the map and the frame defined by the elliptical Gaussian axis. For each source, the fitting procedure has to provide seven parameters: $A$ (the Gaussian amplitude), $\left(x_{s}, y_{s}\right)$ (the position of the source center), $\left(\sigma_{x}, \sigma_{y}\right)$ (the Gaussian widths in two orthogonal directions), $\Theta$ (the rotation angle of the Gaussian axis from the array axis), and an offset value $B_{\text {loc }}$ (the local background for each source).

A real image is not just the sampling of the original signal, of course, but it is the sampling of the convolution of the signal with the PSF of the telescope optics-detector instrument. Using the program STINYTIM, ${ }^{19}$ we obtained theoretical PSFs of the MIPS instrument for both 70 and $160 \mu \mathrm{m}$ bands. Figures 21 and 22 show the theoretical PSFs for the $70 \mu \mathrm{m}$ and $160 \mu \mathrm{m}$ bands and their profiles. In panel (b) of these figures, it is shown also the radial profile of an empirical PSFs (kindly provided by G. Bendo). Although there are some intrinsic differences between empirical and theoretical profiles, we decided to use theoretical PSFs because they can be sampled at any desired rate and do not contain noise that would be added to the fit (however, we made some tests to determine the level of uncertainty introduced by using theoretical PSFs instead of real ones in our fitting procedure. We have found that the difference on the final results is negligible compared to other sources of error, e.g., only $\approx 5 \%$ on the inferred source fluxes).

The first step of our fitting procedure is to assume a distribution of elliptical Gaussian sources, with initial trial values of the parameters, in an array of the same size of the data. First from a direct inspection of the FIR maps and then after some iterations, we identified 10 main sources to be fitted using elliptical Gaussians (see Figure 2): two for NGC 7320 (one for the disk emission plus one for a compact $\mathrm{H}$ II region), one each for SQ A and SQ B, two for NGC 7319 (one for the circumnuclear emission plus one for a $\mathrm{H}$ il region), one for a source located north of NGC 7319 (H II-N), two for NGC $7318 \mathrm{~b}$ (the two compact $\mathrm{H}$ II regions located on the southern spiral arms $\mathrm{H}_{\mathrm{II}} \mathrm{SE}$ and $\mathrm{H}$ II-SW), and one for a source peaked in the middle of the shock region. Convolving this array with a theoretical PSF, an artificial image is obtained that can be compared with the real data. Varying the parameters of the elliptical Gaussians, it is possible to improve the agreement between artificial and real images until a certain degree of accuracy is reached. To minimize $\chi^{2}$ and find the best-fit model parameters, we used the Levemberg-Marquardt (LM) algorithm (see Bevington \& Robinson 1992), implemented by the IDL routine MPFIT2DFUN. ${ }^{20}$ Although this algorithm is less dependent on

\footnotetext{
${ }^{19}$ By John Krist for the Spitzer Science Center: http://ssc.spitzer.caltech.edu/ dataanalysistools/tools/contributed/general/stinytim/.

${ }^{20}$ Written by Craig Markwardt: http://cow.physics.wisc.edu/ craigm/idl/ fitting.html for details on the $\chi^{2}$ minimization routine.
} 

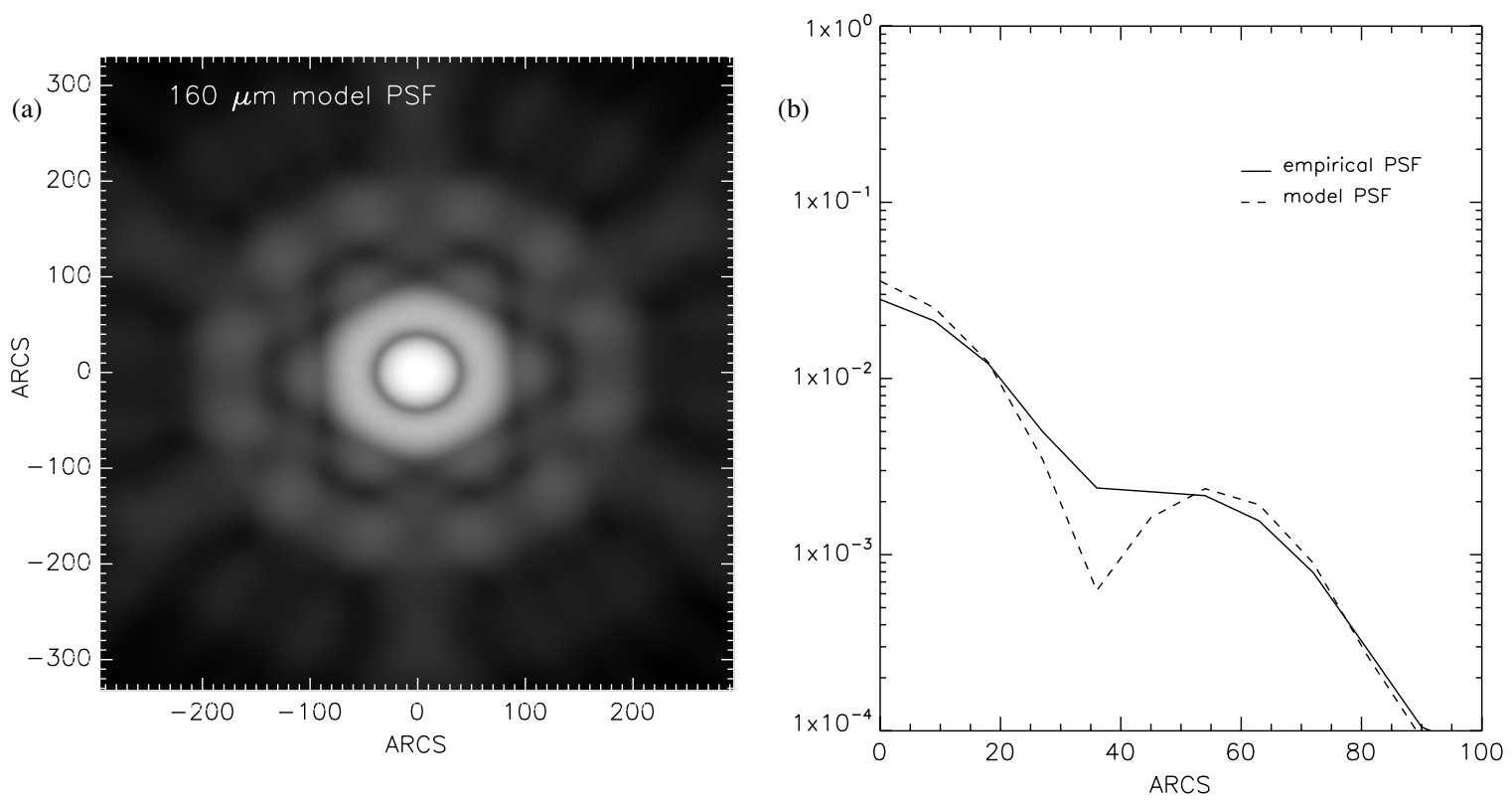

Figure 22. (a) Theoretical $160 \mu \mathrm{m}$ PSF and (b) average radial profiles empirical and theoretical $160 \mu \mathrm{m}$ PSF.

the initial trial values of the parameters, compared with other $\chi^{2}$ minimization methods, the results are inevitably dependent on these. Therefore, it is a good rule to assign the initial values as close as possible to the real ones (or better to which we believe are the real ones). The criteria we used to choose the initial values for the free parameters are the following.

1. Amplitude. Any number of the order of the peak amplitude of the source (this fitted parameter has been found almost independent from the initial value).

2. Position. Initial positions near the peaks of the sources.

3. Gaussian widths. Derived approximately from the apparent width of the source.

4. Rotation angle. Any number.

5. Local background. initial value equal to zero.

As one can notice, the choice of the initial values does not require to know in advance any parameter but the approximate positions of the source centers and roughly the sizes of the Gaussians. All this information can be easily derived from the data. The fitting procedure improves the quality of the results (in the sense that we obtain a smoother fit residual map and a lower reduced $\chi^{2}$ ) if we consider only the regions close to the sources for the fit. We created a mask to exclude all the pixels that are too distant from the calculation, that is more than 2-3 times the apparent sizes, from the source centers.

The fitting technique as described until now has been applied successfully to the $70 \mu \mathrm{m}$ FIR map. The fitting of the $160 \mu \mathrm{m}$ map has been performed in a slightly different way because one cannot clearly see on this map all the compact sources that are detected on the $70 \mu \mathrm{m}$. This effect is most probably due to both lower resolution and intrinsic color differences. Since our goal was to produce consistent SEDs of the main sources in SQ, we introduced new constrains for the fit of the $160 \mu \mathrm{m}$ map. We assumed that the center position of each source on the $160 \mu \mathrm{m}$ map is the same as derived from the $70 \mu \mathrm{m}$ map fitting. We only allowed a common shift of all the source positions in order to correct possible small differences in the astrometry of the two maps. Then we assumed that the orientation angles and the ratio of the elliptical Gaussian axial widths $\left(\sigma_{x}, \sigma_{y}\right)$ for the compact sources (SQ A, H II-SE, H II-SW, SQ B, H II-N, and the $\mathrm{H}$ II regions in NGC 7319 and NGC 7320) are the same as those inferred from the $70 \mu \mathrm{m}$ map fit. Therefore, we assumed that the shape of the emitting compact sources is similar at 70 and $160 \mu \mathrm{m}$ even if the size can be different. This is actually expected because colder emission can come from regions that are simply farther away from the central heating source in the case of star formation regions.

Figures 2 and 3 show the results of our fitting technique for the $70 \mu \mathrm{m}$ and $160 \mu \mathrm{m}$ maps in four panels: (1) the background subtracted Spitzer FIR map of SQ, (2) the best-fit image obtained with our method, (3) the fit residuals, and (4) the "deconvolved" map (that is actually the map showing the elliptical Gaussians whose PSF convolution gives the best-fit map). As one can see, this method produces best-fit maps with remarkable similarity to the original maps $\left(\chi_{70 \mu \mathrm{m}}^{2} / N_{\text {free }}=0.8, \chi_{160 \mu \mathrm{m}}^{2} / N_{\text {free }}=0.7\right)$. The "deconvolved" map at $70 \mu \mathrm{m}$ shows, apart from the emission of the foreground galaxy NGC 7320 and AGN galaxy NGC 7319, many discrete compact sources that are responsible for the several peaks seen on the real map. There is a rather extended source that peaks in the middle of the shock region, even though some excess of emission is still seen on the fit residual map at this position suggesting that the accuracy of the fit is not very good for this faint source. At $160 \mu \mathrm{m}$, the emission is dominated by the AGN galaxy, the foreground galaxy, and the source peaked on the shock region. It is quite interesting that the Gaussian used to fit the emission at the position of the shock is very well aligned along the main axis of the shock ridge. However, the east-west width of this Gaussian is too large to be related only to the X-ray ridge (see Section 4.2). Some compact star formation regions are undetected (or only marginally detected) at $160 \mu \mathrm{m}$, as we somehow expected because no peaks are clearly seen at their position.

The main fitted parameters and the derived flux densities for each of the fitted sources can be found in Table 1. The uncertainties on the integrated fluxes are derived from the covariance matrix provided by the LM $\chi^{2}$ minimization algorithm. For the estimate of the error on each point of the map, necessary to evaluate $\chi^{2}$ we considered the variance of background fluctuations on each map. On the final integrated fluxes for each source a $10 \%$ error is added due to flux calibration uncertainties (MIPS 


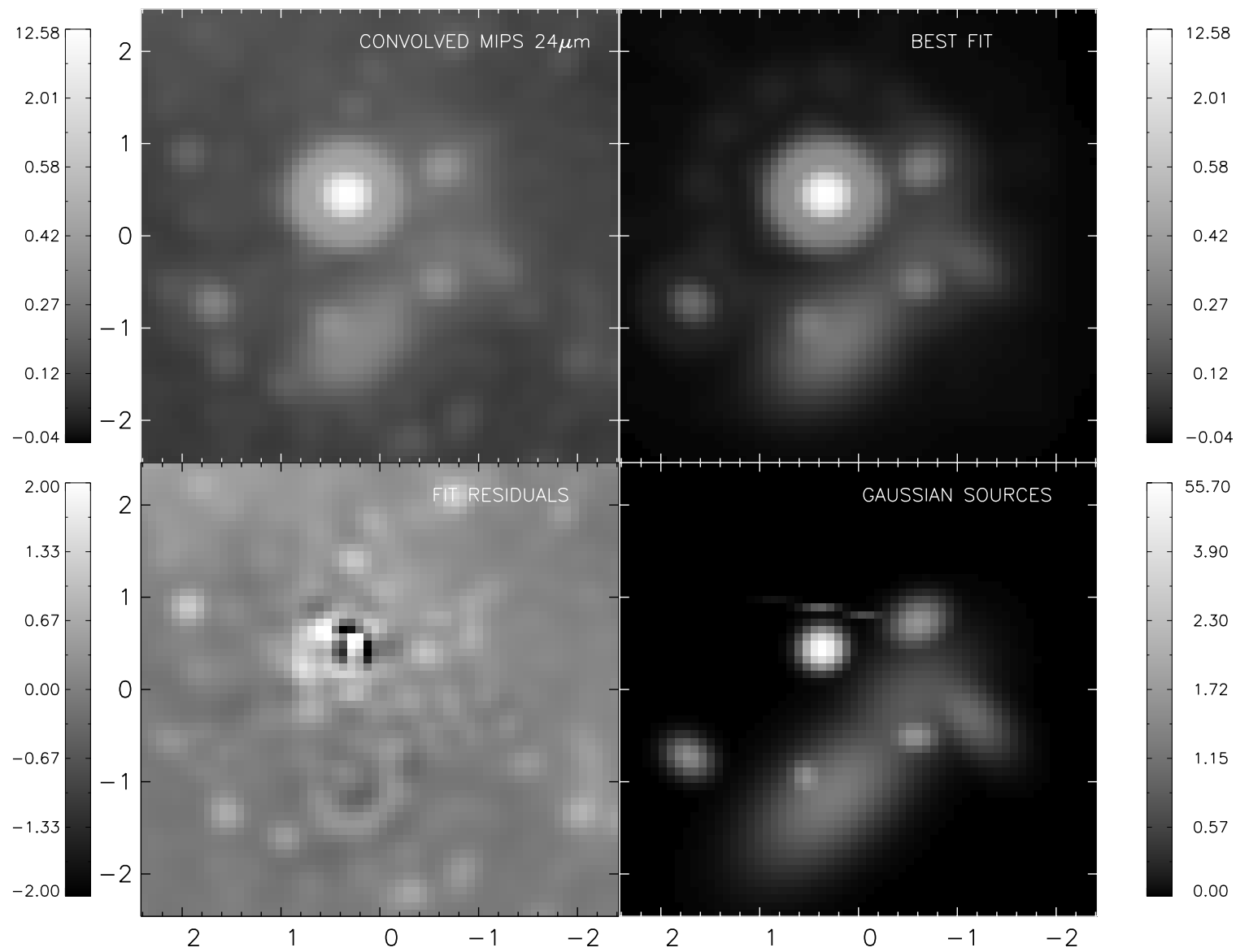

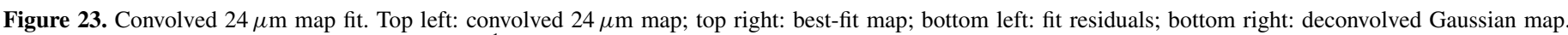
Units of the values aside the color bars are $\mathrm{MJy} \mathrm{sr}^{-1}$.

data handbook). As one can notice from Table 1, the inferred $160 \mu \mathrm{m}$ sizes for the AGN galaxy NGC 7319 and the compact sources $\mathrm{HII-SE}$, SQ B, and $\mathrm{HII}-\mathrm{N}$ are much larger than the $70 \mu \mathrm{m}$ size. In the case of the AGN galaxy, the size difference can be physically understood if one thinks that additional warm emission come from the central part of the galaxy and, therefore, the $70 \mu \mathrm{m}$ flux is more peaked toward the center (see also discussion in Section 7.3). The larger $160 \mu \mathrm{m}$ size for the compact sources arises because of the confusion with fainter sources close to the main central source. For these sources, we derived from the deconvolved $160 \mu \mathrm{m}$ Gaussians the amount of flux within one $70 \mu \mathrm{m}$ FWHM from the peak. These reduced $160 \mu \mathrm{m}$ fluxes are quoted within brackets in Table 2 .

\section{A.1. Comparison between Results Obtained by the Fitting Technique and Aperture Photometry}

Since in Section 5, we performed source SED fitting including MIR and FIR measurements, performed, respectively, using aperture photometry and the map fitting technique, it is important to understand how different the fluxes obtained by the fitting procedure are compared to those we would have obtained by aperture photometry on a high-resolution map. To quantify this difference, we made a test using the higher resolution $24 \mu \mathrm{m}$ map where dust emission morphology looks remarkably similar to the $70 \mu \mathrm{m}$ emission (at least if one considers the main sources of emission). We convolved the $24 \mu \mathrm{m}$ MIR map to match the resolution of the $70 \mu \mathrm{m}$ map, resampled the map to match the pixel size of the $70 \mu \mathrm{m}$ map, and then performed the Gaussian fitting technique on the convolved map. The convolution has been done using the kernel function created by Carl Gordon (Gordon et al. 2008). For the fit we assumed the same distribution of Gaussians as in the fit of the FIR maps, but leaving all the parameters free. The convolved map and the fit results are shown in Figure 23. In Table 6 and Figure 8, we compared the results from aperture photometry (described in Section 4.1) and Gaussian fitting. For all but one source (H II-SW), the fluxes derived using the two different methods are consistent within the uncertainties and also the inferred source sizes are close to those found at $70 \mu \mathrm{m}$ (the problem with $\mathrm{HII-SW}$ arises because of the vicinity of the nucleus of NGC 7318a that is much brighter in the MIR than in the FIR). Based on the results of this test, we are confident that the two photometric techniques give consistent results.

\section{APPENDIX B}

\section{DUST SED TEMPLATES AND MODELS}

In order to fit the observed source SEDs, we needed to model the dust emission from several environments: dust in PDR/H II regions, dust heated by diffuse radiation fields, dust in AGN tori, and collisionally heated dust embedded in hot plasmas. In this section, we describe the characteristics of the SEDs we used to reproduce the emission from each of these regions.

\section{B.1. Dust in PDR/H II Regions}

Dust in H II and PDR, close to young stars, is warm and emits predominantly in the MIR. To model this emission, we used an SED template derived by the fitting of Milky Way star formation 
Table 6

Comparison of Photometry Results at $24 \mu \mathrm{m}$ Obtained with Aperture Photometry and the Gaussian Fitting Technique

\begin{tabular}{lcccc}
\hline \hline \multicolumn{1}{c}{ Source } & $\begin{array}{c}F_{24 \mu \mathrm{m}}^{\mathrm{ap}} \\
(\mathrm{mJy})\end{array}$ & $\begin{array}{c}F_{24 \mu \mathrm{m}}^{\mathrm{fit}} \\
(\mathrm{mJy})\end{array}$ & $\begin{array}{c}\Delta_{70 \mu \mathrm{m}}^{\mathrm{fit}} \\
(\operatorname{arcsec})\end{array}$ & $\begin{array}{c}\Delta_{24 \mu \mathrm{m}}^{\mathrm{fit}} \\
(\operatorname{arcsec})\end{array}$ \\
\hline SQ A & $11 \pm 2$ & $12 \pm 0.5$ & 16 & 15 \\
H II-SE & $7.5 \pm 1$ & $6.7 \pm 0.4$ & 14 & 12 \\
H II-SW $^{\mathrm{a}}$ & $2.4 \pm 0.35$ & $6 \pm 0.6$ & 11 & 24 \\
SQ B & $5.9 \pm 0.8$ & $5.6 \pm 0.8$ & 15 & 12 \\
NGC 7319 $^{\mathrm{b}}$ & $185 \pm 8$ & $195 \pm 8$ & 12 & 12 \\
NGC 7320 & $38 \pm 2$ & $40 \pm 2$ & 44 & 43 \\
H II-N & $1.1 \pm 0.2$ & $\mathrm{ND}^{\mathrm{c}}$ & 22 & $\mathrm{ND}^{\mathrm{c}}$
\end{tabular}

Notes. Column 1: source name; Column 2: aperture photometry fluxes; Column 3: fluxes from the Gaussian fitting technique; Column 4: average source FWHMs as derived from the $70 \mu \mathrm{m}$ map fitting; Column 5: average source FWHMs as derived from the $24 \mu \mathrm{m}$ convolved map fit.

a This source is the only source, detected by the fitting technique, whose inferred fluxes are considerably different. The size of the Gaussian that fit the emission on the $24 \mu \mathrm{m}$ convolved map is much larger than that found at $70 \mu \mathrm{m}$. The reason for this is the contamination from the nearby nucleus of NGC 7318a that emits strongly in the MIR but not in the FIR.

b For NGC 7319 (the AGN galaxy) and NGC 7320 (the foreground galaxy), the apertures are chosen such to cover the entire emission of the galaxies. In the fitting procedure, these sources are modeled with two Gaussians, one for the centrally peaked emission and the other for a peripherical $\mathrm{H}$ II region. The sizes shown in Columns 4 and 5 refer to the central source that contribute most of the flux.

c ND: non-detected.

region infrared emission with the theoretical model of Groves et al. (2008). The Galactic star formation regions considered are the radio-selected sample of Conti \& Crowther (2004). The template is the average fitted spectra of the observed emission from these regions. Further details will be given in a forthcoming paper (Popescu et al. 2010, Section 2.9). The template is shown in the left panel of Figure 11.

\section{B.2. Dust Heated by Diffuse Radiation Fields}

To fit the diffuse dust emission, we created a grid of theoretical SEDs of emission from dust heated by a uniform radiation field. The code we used to calculate dust emission, developed by Joerg Fischera (details will be given in J. Fischera et al. 2010, in preparation), assumes a dust composition including graphite, silicates, iron, and PAH molecules. The abundances and size distributions are the same as in Table 2 of Fischera \& Dopita (2008). Dust emission is calculated taking into account the stochastic temperature fluctuations of dust grains following the numerical method described by Guhathakurta \& Draine (1989), combined with the step wise analytical solution of Voit (1991). We created the set of SEDs by varying the intensity and the color of the radiation field heating the dust and keeping the dust composition fixed. The standard shape of the radiation field spectra, adopted by the dust emission code, is that derived by Mathis et al. (1983) to model the Galactic local ISRF. In this spectrum, the stellar contribution consists of four components: a UV emission from early-type stars plus three blackbody curves at temperatures $T_{2}=7500 \mathrm{~K}, T_{3}=4000 \mathrm{~K}$, and $T_{4}=3000 \mathrm{~K}$ used to reproduce, respectively, the emission from young/old disk stars and red giants. Each blackbody curve is multiplied by a dilution factor $W_{i}$ that defines its intensity. The whole spectrum is multiplied by a parameter, $\chi_{\text {isrf }}$, whose value is unity when the spectrum intensity is the same as in the local radiation field. In order to consider different colors of the radiation field, we defined a second parameter, $\chi_{\text {color }}$, that multiplies only the blackbody curves used to model the old disk population and the red giant emission (blackbody temperatures $T_{3}$ and $T_{4}$ ). For a range of values of $\chi_{\text {isrf }}(0.1,0.3,0.5,1,2,4)$ and $\chi_{\text {color }}(0.25$, $0.5,1,2,4)$, we calculated the corresponding dust emission and created the set of models for the SED fit. The result of increasing the $\chi_{\text {isrf }}$ value is that the overall intensity of the radiation field is higher and, therefore, the equilibrium dust temperature is higher, leading to a warmer emission in the FIR (see the left panel of Figure 12). A value of $\chi_{\text {color }}$ higher than unity increases the relative contribution of the cold stellar emission, which peaks in the optical range. These wavelengths are more efficiently absorbed by big grains than by PAH molecules (and small grains). As a consequence, by varying this parameter, one modifies the ratio between the peak intensity at the FIR, produced by big grains and the peak intensity at $8 \mu \mathrm{m}$, due to PAH molecules which are mainly heated by UV photons (see the right panel of Figure 12). A similar effect would have also been produced by varying the relative abundance of PAH molecules and solid grains.

\section{B.3. Dust Emission from AGN Torus}

We selected a theoretical dusty torus SED between those created by A. Feltre et al. (2010, in preparation), using the model presented by Fritz et al. (2006). In this model, the characteristic of the torus, supposed to be homogeneous, is defined by several parameters: opening angle, external to internal radius ratio, equatorial optical depth at $9.7 \mu \mathrm{m}$ and two parameters, $\beta$ and $\gamma$, that determine the radial and the angular density profile, respectively, according to the formula $\rho(r, \theta)=\alpha r^{-\beta} e^{-\gamma|\cos (\theta)|}$, where $r$ is the radial coordinate and $\theta$ is the angle between a volume element and the equatorial plane (note that $\alpha$ is determined by the torus optical depth). We selected a model with a large opening angle $\left(140^{\circ}\right)$, with an angular gradient in the density profile $(\beta=0, \gamma=6)$, external to internal radius ratio equal to 30 and equatorial optical depth $\tau(9.7 \mu \mathrm{m})=6$. The chosen parameters are well inside the range of values found by Fritz et al. (2006) from the fitting of a sample of Seyfert 2 galaxies. Then, we extracted the output SED in the equatorial view, according to the Seyfert 2 classification of NGC 7319, the AGN galaxy in SQ. The extracted SED is the template we used for fitting the AGN torus emission and it is shown in the right panel of Figure 11.

\section{B.4. Dust Heated by Collisions in X-ray Plasmas}

Finally, we needed to model the emission from collisionally heated dust. For this purpose, we used a code, created by J. Fischera, based on the works by Guhathakurta \& Draine (1989) and Voit (1991) for calculating the stochastical heating of the dust grains and using the results of Draine \& Salpeter (1979) and Dwek (1987) to determine the heating rates due to dust-plasma particle collisions. Dust composition is assumed to be a mix of graphite $(47 \%)$ and silicates $(53 \%)$. PAH molecules are not included but, since they have an extremely small destruction timescale when embedded in hot plasmas with $T>10^{6} \mathrm{~K}$, their abundance is expected to be negligible. In order to perform the calculations, one needs to specify the physical properties of the plasma, i.e., density, temperature, and metallicity. The exact value of metallicity is not important because dust is predominantly heated by collisions with electrons, provided mainly by ionized hydrogen and helium. Plasma temperature and density are derived from X-ray data (see Appendix C). An important 
Table 7

X-ray Gas Parameters for the Shock and the Halo Components

\begin{tabular}{lccccc}
\hline \hline Source & $\begin{array}{c}Z \\
\left(Z_{\odot}\right)\end{array}$ & $\begin{array}{c}\Omega \\
\left(10^{-23} \mathrm{erg} \mathrm{cm}^{3} \mathrm{~s}^{-1}\right)\end{array}$ & $\begin{array}{c}\text { los } / R \\
(\mathrm{kpc})\end{array}$ & $\begin{array}{c}n \\
\left(\mathrm{~cm}^{-3}\right)\end{array}$ & $\begin{array}{c}M_{\text {gas }} \\
\left(10^{9} M_{\odot}\right)\end{array}$ \\
\hline SHOCK & 1 & 3.82 & 5 & 0.012 & 0.65 \\
SHOCK & 1 & 3.82 & 10 & 0.009 & 0.92 \\
SHOCK & 1 & 3.82 & 20 & 0.006 & 1.30 \\
SHOCK & 1 & 3.82 & 40 & 0.004 & 1.84 \\
SHOCK & 0.3 & 1.92 & 5 & 0.018 & 0.92 \\
SHOCK & 0.3 & 1.92 & 10 & 0.012 & 1.30 \\
SHOCK & 0.3 & 1.92 & 20 & 0.009 & 1.83 \\
SHOCK & 0.3 & 1.92 & 40 & 0.006 & 2.60 \\
HALO & 1 & 2.54 & 40 & 0.0011 & 10.4 \\
HALO & 0.3 & 1.39 & 40 & 0.0016 & 14.4 \\
\hline
\end{tabular}

Notes. Column 1: source; Column 2: assumed metallicity; Column 3: cooling rate; Column 4: line-of-sight depth (for the shock), or sphere radius (for the halo); Column 5: gas number density defined as $n=\left(n_{t} n_{e}\right)^{1 / 2}$; Column 6: gas mass.

phenomenon that should be taken into account while preparing the SED models is dust destruction due to sputtering (Draine \& Salpeter 1979). Because the efficiency of sputtering depends on the grain size, we expect that the size distribution of grains in hot plasmas is different from that of dust in the cold ISM, typically assumed to be $n(a) \propto a^{-k}$ with $k=3.5$ (Mathis et al. 1977). Therefore, we created a set of SEDs varying (1) the plasma physical parameters (see Table 7) and (2) the exponent $k$ of the size distribution in the range $[1,3.5]$. A $k$ value lower than 3.5 means that the relative number of big grains is higher compared to the standard size distribution. Since the destruction timescale due to dust sputtering is directly proportional to the grain size (Draine \& Salpeter 1979), a higher relative abundance of big grains is indeed expected in hot plasmas. Once the heating rates due to collisions are fixed, a change in $k$ modifies the color of the emitted radiation (see Figure 13). This happens because big grains are generally colder than small grains, and do not provide warm dust emission due to stochastic heating. Therefore, changing their relative abundance, one can obtain colder or warmer SEDs.

\section{APPENDIX C}

\section{X-RAY PLASMA TEMPERATURE AND DENSITY IN SQ SHOCK AND HALO}

As we mentioned in Appendix B.4, the hot plasma physical parameters, needed for the calculation of the emission SED from collisionally heated dust, in both the shock and halo gas, are derived from the X-ray emission. The temperatures characteristic of the plasma have been obtained by Trinchieri et al. (2005) fitting XMM-NETWON X-ray data and quoted in their Table 3. They fitted the source X-ray spectra with two temperature plasma models. The fitted colder component is predominant for the shock region and has a temperature $T \approx 3 \times 10^{6} \mathrm{~K}$ that we assumed to calculate the dust emission. For the halo region, the two plasma components have similar luminosities. In this case, we assumed the average value $T=6 \times 10^{6} \mathrm{~K}$ for the dust emission calculation (the fitted temperatures are $T_{1}=3.5 \times 10^{6} \mathrm{~K}$ and $T_{2}=8.8 \times 10^{6} \mathrm{~K}$ ). The plasma densities have been derived from the X-ray luminosity, which can be expressed as

$$
L=n_{t} n_{e} \Lambda(T) V
$$

where $n_{t}$ and $n_{e}$ are the ion and the electron number densities, respectively, $\Lambda(T)$ is the cooling function, and $V$ is the volume of the X-ray-emitting plasma. From a given $L, \Lambda(T)$, and $V$, one can calculate the gas density $n=\left(n_{t} n_{e}\right)^{0.5}$ by inverting Equation (C1). Given the plasma temperatures found by Trinchieri et al. (2005), we derived $\Lambda(T)$ by interpolating the collisional ionization equilibrium (CIE) curve calculated by Sutherland \& Dopita (1993) at two different metallicities corresponding to $100 \%$ and $30 \%$ the solar metallicity. Then, we assumed for the shock gas an emitting volume equal to the projected shock ridge area $\left(A=330 \mathrm{kpc}^{2}\right)$ multiplied by a parameterized line-of-sight depth $l$, and for the halo we adopted an emitting sphere of radius $40 \mathrm{kpc}$, corresponding to $90^{\prime \prime}$ at $94 \mathrm{Mpc}$ distance. The luminosity values we used are those inferred by Trinchieri et al. (2005) $\left(L_{\mathrm{X}}^{\text {shock }} \approx 2.4 \times 10^{41} \mathrm{erg} \mathrm{s}^{-1}\right.$ and $L_{\mathrm{X}}^{\text {halo }} \approx 2.3 \times 10^{41} \mathrm{erg} \mathrm{s}^{-1}$ ), properly scaled to the different assumed distance (85 Mpc in that paper versus $94 \mathrm{Mpc}$ here). The results for different choices of the emitting volumes and metallicity are shown in Table 7 . For the shock region plasma, we obtained densities in the range (4-18) $\times 10^{-3} \mathrm{~cm}^{-3}$. As expected, the minimum density is obtained for higher metallicity (that is, higher $\Lambda$ ) and largest volume, while the maximum density corresponds to lower metallicity (lower $\Lambda$ ) and smallest volume. For the halo gas, the inferred densities are $1.1 \times 10^{-4}$ and $1.6 \times 10^{-4} \mathrm{~cm}^{-3}$ for $Z=Z_{\odot}$ and $Z=0.3 Z_{\odot}$, respectively.

\section{APPENDIX D}

\section{IRAC AND MIPS COLOR CORRECTIONS}

From the IRAC and MIPS data handbook, the color corrections for each Spitzer band are defined as

$$
\begin{gathered}
K=\frac{\int\left(F_{v} / F_{v o}\right)\left(v / v_{o}\right)^{-1} R d v}{\int\left(\nu / v_{o}\right)^{-2} R d v}(\text { IRAC }), \\
K=\frac{\int \frac{F_{\lambda}}{F_{\lambda o}} R_{\lambda} d \lambda}{\int\left(\frac{\lambda_{o}}{\lambda}\right)^{5} \frac{e^{\frac{h c}{\lambda o k T_{o}}}-1}{e^{\frac{h c}{\lambda K T_{o}}}-1} \lambda R_{\lambda}}(\mathrm{MIPS}),
\end{gathered}
$$

where the subscripts $v_{o}$ and $\lambda_{o}$ refer to the band reference frequency or wavelength, $R$ is the instrument spectral response, and $T_{o}=10.000 \mathrm{~K}$ (these formulae derive from the conventions used to calculate the quoted fluxes on the Spitzer maps).

\section{APPENDIX E}

\section{ESTIMATE OF AN UPPER LIMIT TO THE DUST INJECTION RATE FROM HALO STARS IN SQ}

An upper limit to the dust injection rate from halo stars in SQ can be inferred from the halo $R$-band surface brightness, $S_{\mathrm{R}}=$ 24.4mag $\operatorname{arcsec}^{-2}$ (Moles et al. 1998), and using theoretical predictions for the stardust injection. We assumed that all the $R$-band halo luminosity is produced by $3 M_{\odot}$ stars. According to Table A.1 in Zhukovska et al. (2008), a $3 M_{\odot}$ star with metallicity $Z=0.02$ injects $M_{\mathrm{d}}=1.2 \times 10^{-2} M_{\odot}$ of dust during its all life. Given the lifetime of such star, $\tau_{\text {life }} \approx 3 \times 10^{8} \mathrm{yr}$, one can derive the average dust injection rate: $M_{\mathrm{d}}=M_{\mathrm{d}} / \tau_{\text {life }}=$ $3.5 \times 10^{-11} M_{\odot} \mathrm{yr}^{-1}$. Since a star spends most of its lifetime in the main-sequence phase, we approximate the $R$-band flux of a single halo star as that of a blackbody sphere with parameters characteristic of a main-sequence $3 M_{\odot}$ star:

$$
F_{\mathrm{R}}=\pi B_{\mathrm{R}}(T) \frac{R^{2}}{d^{2}}
$$


where $B_{\mathrm{R}}(T)$ is the blackbody $R$-band flux density at temperature $T=12,000 \mathrm{~K}, R=2 R_{\odot}$ is the star radius, and $d=$ $94 \mathrm{Mpc}$ is the distance from SQ. The prediction for the $R$-band flux density produced by a single halo star and observed from the earth is $F_{\mathrm{R}}=1.35 \times 10^{-23} \mathrm{erg} \mathrm{cm}^{-2} \mathrm{~s}^{-1} \mathrm{~A}^{-1}$. Dividing $\dot{M}_{\mathrm{d}}$ to $F_{\mathrm{R}}$, we obtain the dust injection rate per observed $R$-band flux density: $\dot{M}_{\mathrm{d}} / F_{\mathrm{R}}=2.6 \times 10^{12} M_{\odot} \mathrm{yr}^{-1}\left(\mathrm{erg} \mathrm{cm}^{-2} \mathrm{~s}^{-1} \mathrm{~A}^{-1}\right)^{-1}$. Combining the observed $R$-band surface brightness $S_{\mathrm{R}}$ and the solid angle $\Omega$ covering the halo region of SQ, one can obtain the total received flux $F_{\mathrm{R}}^{\mathrm{obs}}=2.9 \times 10^{-14} \mathrm{erg} \mathrm{cm}-2 \mathrm{~s}^{-1} \mathrm{~A}^{-1}$. Then the dust injection rate from halo stars is equal to

$$
\dot{M}_{\mathrm{d}}=\left(\frac{\dot{M}_{\mathrm{d}}}{F_{\mathrm{R}}}\right) F_{\mathrm{R}}^{\mathrm{obs}} \text {. }
$$

Using this simple approach we found $\dot{M}_{\mathrm{d}}=0.075 M_{\odot} \mathrm{yr}^{-1}$. This value should be considered as an upper limit since one can show, using the theoretical predictions for low-intermediate mass star dust injection rates in Zhukovska et al. (2008), that $3 M_{\odot}$ stars have the highest value of the dust injection rate per $R$-band flux $\dot{M}_{\mathrm{d}} / F_{\mathrm{R}}$.

\section{REFERENCES}

Aoki, K., Kosugi, G., Wilson, A. S., \& Yoshida, M. 1999, ApJ, 521, 565

Appleton, P. N., et al. 2006, ApJ, 639, 51

Bai, L., Rieke, G. H., \& Rieke, M. J. 2007, ApJ, 668, 5

Bell, E. F., McIntosh, D. H., Katz, N., \& Weinberg, M. D. 2003, ApJS, 149, 289

Bendo, G. J., et al. 2008, MNRAS, 389, 629

Bevington, P. R., \& Robinson, D. K. 1992, Data Reduction and Error Analysis for the Physical Sciences (New York: McGraw-Hill)

Bigiel, F., et al. 2008, AJ, 136, 2846

Blandford, R., \& Eichler, D. 1987, Phys. Rep., 154, 1

Boquien, M., et al. 2009, AJ, 137, 4561

Braine, J., et al. 2001, A\&A, 378, 51

Brinchmann, J., Charlot, S., White, S. D. M., \& Tremonti, C. 2004, MNRAS, 351,1151

Calzetti, D., et al. 2007, ApJ, 666, 870

Cavaliere, A., \& Lapi, A. 2008, ApJ, 673, 5

Chelouche, D., Koester, B. P., \& Bowen, D. V. 2007, ApJ, 671, 97

Cluver, M. E., et al. 2010, ApJ, 710, 248

Conti, P. S., \& Crowther, P. A. 2004, MNRAS, 355, 899

Cortese, L., et al. 2010, A\&A, 518, 63

Dekel, A., \& Birnboim, Y. 2006, MNRAS, 368, 2

Dopita, M. A., et al. 2005, ApJ, 619, 755

Draine, B. T., \& Salpeter, E. E. 1979, ApJ, 231, 77

Driver, S. P., et al. 2009, Astron. Geophys., 50, 12

Dwek, E. 1987, ApJ, 322, 812

Dwek, E., Rephaeli, Y., \& Mather, J. C. 1990, ApJ, 350, 104

Eke, V. R., Baugh, C. M., Cole, S., Frenk, C. S., King, H. M., \& Peacock, J. A. 2005, MNRAS, 362, 1233

Fazio, G. G., et al. 2004, ApJS, 154, 10

Fischera, J., \& Dopita, M. A. 2008, ApJS, 176, 164

Fischera, J., Tuffs, R. J., \& Völk, H. J. 2002, A\&A, 395, 189

Frenk, C. S., et al. 1999, ApJ, 525, 554

Fritz, J., Franceschini, A., \& Hatziminaoglou, E. 2006, MNRAS, 366, 767

Gallagher, S. C., Charlton, J. C., Hunsberger, S. D., \& Zaritsky, D. 2001, AJ, 122,163

Gao, Y., \& Xu, C. 2000, ApJ, 542, 83

Giard, M., Montier, L., Pointecouteau, E., \& Simmat, E. 2008, A\&A, 490, 547

Gordon, K. D., Engelbracht, C. W., Rieke, G. H., \& Misselt, K. A. 2008, ApJ, 682,336
Green, D. A., Tuffs, R. J., \& Popescu, C. C. 2004, MNRAS, 355, 1315

Groves, B., Dopita, M. A., Sutherland, R. S., Kewley, L. J., Fischera, J., Leitherer, C., Brandl, B., \& van Breugel, W. 2008, ApJS, 176, 438

Guhathakurta, P., \& Draine, B. T. 1989, ApJ, 345, 230

Guillard, P., Boulanger, F., Pineau Des Forêts, G., \& Appleton, P. N. 2009, A\&A, 502,515

Guillard, P., Boulanger, F., Cluver, M. E., Appleton, P. N., Pineau Des Forêts, G., \& Ogle, P. 2010, A\&A, 518, 59

Helou, G., et al. 2004, ApJS, 154, 253

Huchra, J. P., Wyatt, W. F., \& Davis, M. 1982, AJ, 87, 1628

Kennicutt, R. C. 1998, ARA\&A, 36, 189

Kennicutt, R. C., et al. 2007, ApJ, 671, 333

Kitayama, T., et al. 2009, ApJ, 695, 1191

Leon, S., et al. 2008, A\&A, 485, 475

Lisenfeld, U., Braine, J., Duc, P.-A., Leon, S., Charmandaris, V., \& Brinks, E. 2002, A\&A, 394, 823

Mathis, J. S., Mezger, P. G., \& Panagia, N. 1983, A\&A, 128, 212

Mathis, J. S., Rumpl, W., \& Nordsieck, K. H. 1977, ApJ, 217, 425

Micelotta, E. R., Jones, A. P., \& Tielens, A. G. G. M. 2010, A\&A, 510, 37

Moles, M., Marquez, I., \& Sulentic, J. W. 1998, A\&A, 334, 473

Montier, L. A., \& Giard, M. 2004, A\&A, 417, 401

Mulchaey, J. S. 2000, ARA\&A, 38, 289

Nakagawa, T. 2010, Proc. SPIE, 7731, 773100

Natale, G. 2010, PhD thesis, Reprecht-Karls-Universität Heidelberg

O’Sullivan, E., Giacintucci, S., Vrtilek, J. M., Raychaudhury, S., \& David, L. P. 2009, ApJ, 701, 1560

Pierini, D., Popescu, C. C., Tuffs, R. J., \& Völk, H. J. 2003, A\&A, 409, 907

Popescu, C. C., Misiriotis, A., Kylafis, N. D., Tuffs, R. J., \& Fischera, J. 2000, A\&A, 362, 138

Popescu, C. C., \& Tuffs, R. J. 2002, MNRAS, 335, 41

Popescu, C. C., \& Tuffs, R. J. 2010, in AIP Conf. Proc. 1240, Hunting for the Dark: The Hidden Side of Galaxy Formation, Malta, ed. V. P. Debattista \& C. C. Popescu (Melville, NY: AIP), 35

Popescu, C. C., Tuffs, R. J., Fischera, J., \& Völk, H. 2000, A\&A, 354, 480

Popescu, C. C., et al. 2010, A\&A, in press (arXiv:1011.2942)

Quillen, A. C., Rieke, G. H., Rieke, M. J., Caldwell, N., \& Engelbracht, C. W. 1999, ApJ, 518, 632

Rasmussen, J., Ponman, T. J., Verdes-Montenegro, L., Yun, M. S., \& Borthakur, S. 2008, MNRAS, 388, 1245

Rieke, G. H., et al. 2004, ApJS, 154, 25

Roncarelli, M., Pointecouteau, E., Giard, M., Montier, L., \& Pello, R. 2010, A\&A, 512, 20

Salim, S., Rich, R. M., Charlot, S., \& Brinchmann, J. 2007, ApJS, 173, 267

Schlegel, D. J., Finkbeiner, D. P., \& Davis, M. 1998, ApJ, 500, 525

Springel, V., et al. 2005, Nature, 435, 629

Stickel, M., Klaas, U., Lemke, D., \& Mattila, K. 2002, A\&A, 383, 367

Stickel, M., Lemke, D., Mattila, K., Haikala, L. K., \& Haas, M. 1998, A\&A, 329,55

Sulentic, J. W., Rosado, M., Dultzin-Hacyan, D., Verdes-Montenegro, L., Trinchieri, G., Xu, C., \& Pietsch, W. 2001, AJ, 122, 2993

Sutherland, R. S., \& Dopita, M. A. 1993, ApJS, 88, 253

Swinyard, B. M., et al. 2009, ExA, 23, 193

Tran, K. H., Saintonge, A., Moustakas, J., \& Bai, L. 2009, ApJ, 705, 809

Trinchieri, G., Sulentic, J., Pietsch, W., \& Breitschwerdt, D. 2005, A\&A, 444 697

Vasudevan, R. V., Fabian, A. C., Gandhi, P., Winter, L. M., \& Mushotzky, R. F. 2010, MNRAS, 402, 1081

Verdes-Montenegro, L., Yun, M. S., Williams, B. A., Huchtmeier, W. K., Del Olmo, A., \& Perea, J. 2001, A\&A, 377, 812

Voit, G. M. 1991, ApJ, 379, 122

Williams, B. A., Yun, M. S., \& Verdes-Montenegro, L. 2002, AJ, 123, 2417

Wyder, T. K., Martin, D. C., \& Schiminovich, D. 2007, ApJS, 173, 293

Xu, C. K., Buat, V., Iglesias-Páramo, J., \& Takeuchi, T. T. 2006, ApJ, 646, 834

Xu, C. K., Iglesias-Páramo, J., Burgarella, D., \& Rich, R. M. 2005, ApJ, 619, 95

Xu, C. K., Lu, N., Condon, J. J., Dopita, M., \& Tuffs, R. J. 2003, ApJ, 595, 665

Xu, C. K., Sulentic, J. W., \& Tuffs, R. J. 1999, ApJ, 512, 178

Zhukovska, S., Gail, H.-P., \& Trieloff, M. 2008, A\&A, 479, 453 